NuN

$\sqrt{ })$

1) 8

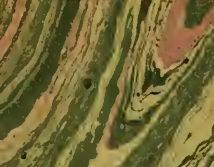

1)

-

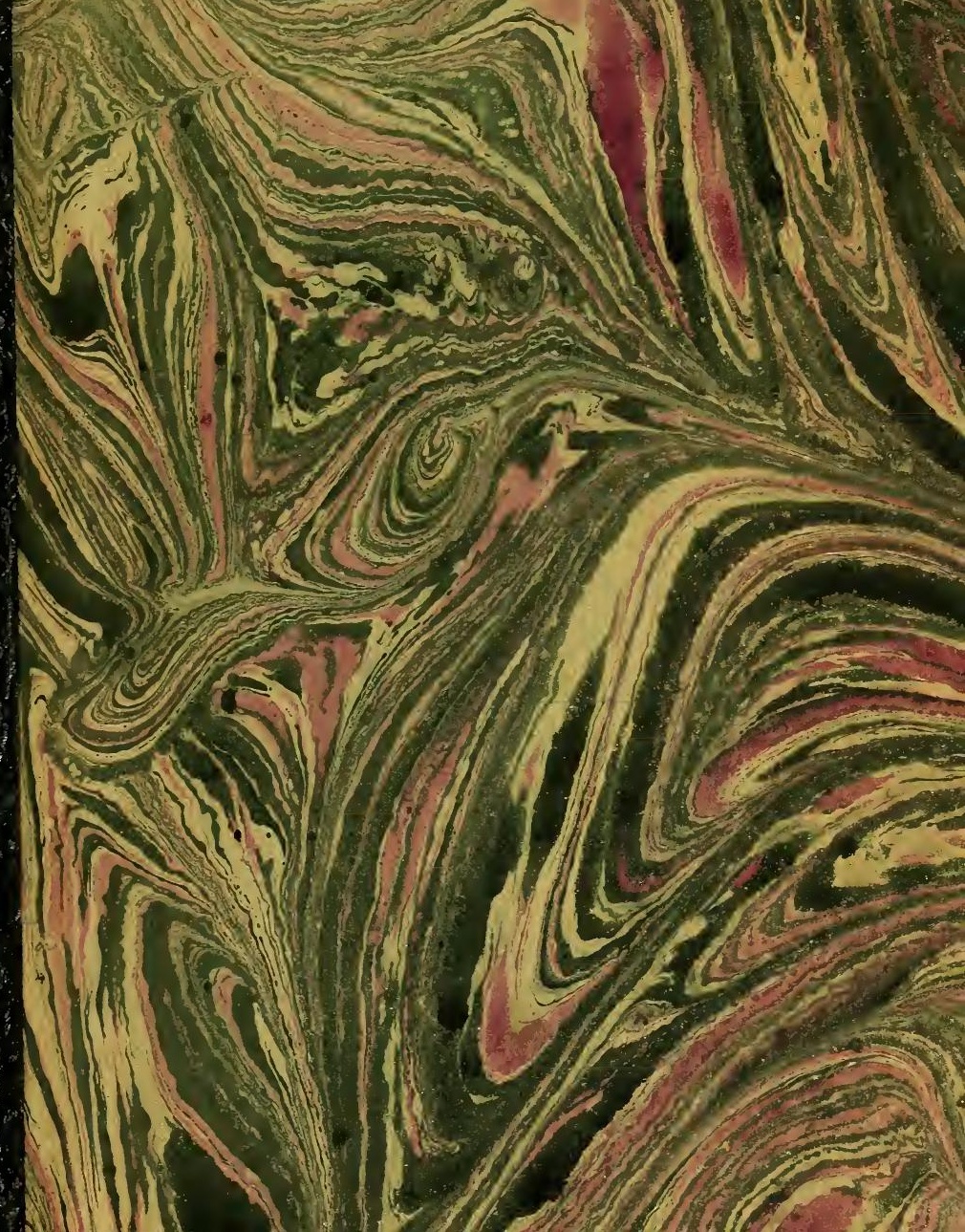










\section{Le Congo Belge}

Notes et Impressions 



\section{René Vauthier}

\section{Le Congo Belge}

\section{Notes et Impressions}

Ouvrage orné de 27 phototypies hors texte
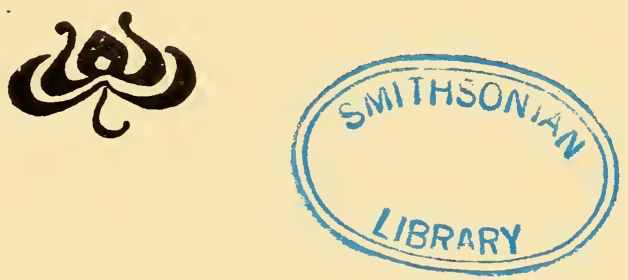

BRUXELLES

J. LEBĖGUE \& Cie, ÉditeurS

Office de Publicité

46, rue de la Madeleine.

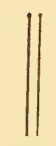

\section{PARIS}

AUgustin CHALLAMEL, Éditeur

I7, rue Jacob.

Librairie $\mathrm{M}$ aritime et Coloniale.

I 900 
Bruxelles. - Imp. Ve Monirom, 32, rue de l'Industrie. 


\section{Dédié}

it Son Altesse Royale

Monseigneur le Prince Albert de Belgique

avec son autorisation 



\section{AVANT-PROPOS}

Un peu partout l'on a encore présente à l'esprit l'excursion de l'Albertville qui aboutit, en juillet I 898 , à l'inauguration du chemin de fer de Matadi au Stanley-Pool. Quelques privilégiés, détachés du groupe des passagers, eurent la bonne fortune, après les fêtes, d'être invités par l'État à pousser jusqu'en plein centre africain, en remontant le Congo jusqu'aux chutes de Stanley.

L'auteur de ces notes et impressions fut de l'un et de l'autre itinéraire. Ayant franchi le seuil du bas fleuve et traversé le vestibule d'entrée de la région des cataractes, il eut le loisir de visiter l'intérieur. En 
cela, il n'accomplit rien de notoire; au contraire, le caractère le plus marquant de son entreprise est d'avoir été extrêmement commode et facile, voire confortable. Or, il $y$ a quelque vingt ans, la descente $d u$ Congo, effectuée par l'illustre Stanley, ressemblait à une épopée. L'extraordinaire ici, c'est donc, à époques si rapprochées, l'énorme différence qui sépare le touriste de l'explorateur, sur une route cependant identique.

La raison du changement? Mais c'est cela qui mérite, semble-t-il, d'être exposé. Non pas à grand renfort d'arguments, mais à l'aide de souvenirs empruntés à la vivante réalité. La chose vue, touchée du doigt, pour ainsi dire, formera comme la trame d'une esquisse dont la sincérité ne saurait en aucun cas être suspectée.

Personne d'ailleurs ne s'attend à yoir un simple témoin tenter la description complète du Congo, embrasser d'un coup d'œil sa constitution physique, découvrir la société indigène, déterminer la valeur politique et économique de la future colonie. A cette fin il faudrait instituer une patiente enquête et la poursuivre pendant de longues années. Mais il est à la portée de qui veut $\mathcal{Y}$ prêter attention, de saisir dans ce vaste ensemble quelques traits essentiels et de chercher à en dégager la signification. N'était l'abus du mot, c'est peut-être bien le "milieu " qui surgirait alors des observations recueillies au jour le jour : un milieu 
spécial qui est la résultante de l'action, en pleine barbarie, des hommes civilisés, des blancs, et de la réaction des sauvages, des nègres du continent mystérieux.

Pour élucider les questions qui touchent à ce point de vue nettement défini, le voyage de Léopoldville à Stanleyville est des mieux appropriés. Le long du fleuve s'échelonnent des populations autochtones qui ont conservé leur entière originalité, et de même c'est par cette grande artère du corps congolais que s'est opérée la première transfusion des forces nouvelles venues d'Europe. La juxtaposition, la combinaison et, en une certaine mesure, l'association de ces éléments hétérogènes, n'est-ce point, en dernière analyse, le fond de la colonisation, et, en particulier, l'explication de cette création hautement intéressante qu'est l'État Indépendant?

Des guides d'une compétence éprouvée ont beaucoup facilité cet essai de reconnaissance d'ordre colonial. Ce furent, à tous les degrés de la hiérarchie, les fonctionnaires et les agents de l'État, et aussi les missionnaires, les commerçants qui partout, avec infiniment de complaisance, montrèrent, renseignèrent, firent part des souvenirs tirés de leur solide expérience personnelle. A défaut de leurs noms, il sera permis de n'évoquer ici que les sentiments d'amitié et de gratitude qui leur sont dus. Enfin, là où la plume ne traduirait qu'imparfaitement telle ou telle leçon de choses, il convient encore de remercier des collaborateurs 
fidèles et sûrs, les appareils photographiques qùe manièrent des mains expertes.

Quant à l'auteur, il estimera avoir réussi dans sa tâche si on lui sait gré de son souci d'exactitude et de la franchise de ses convictions coloniales. 


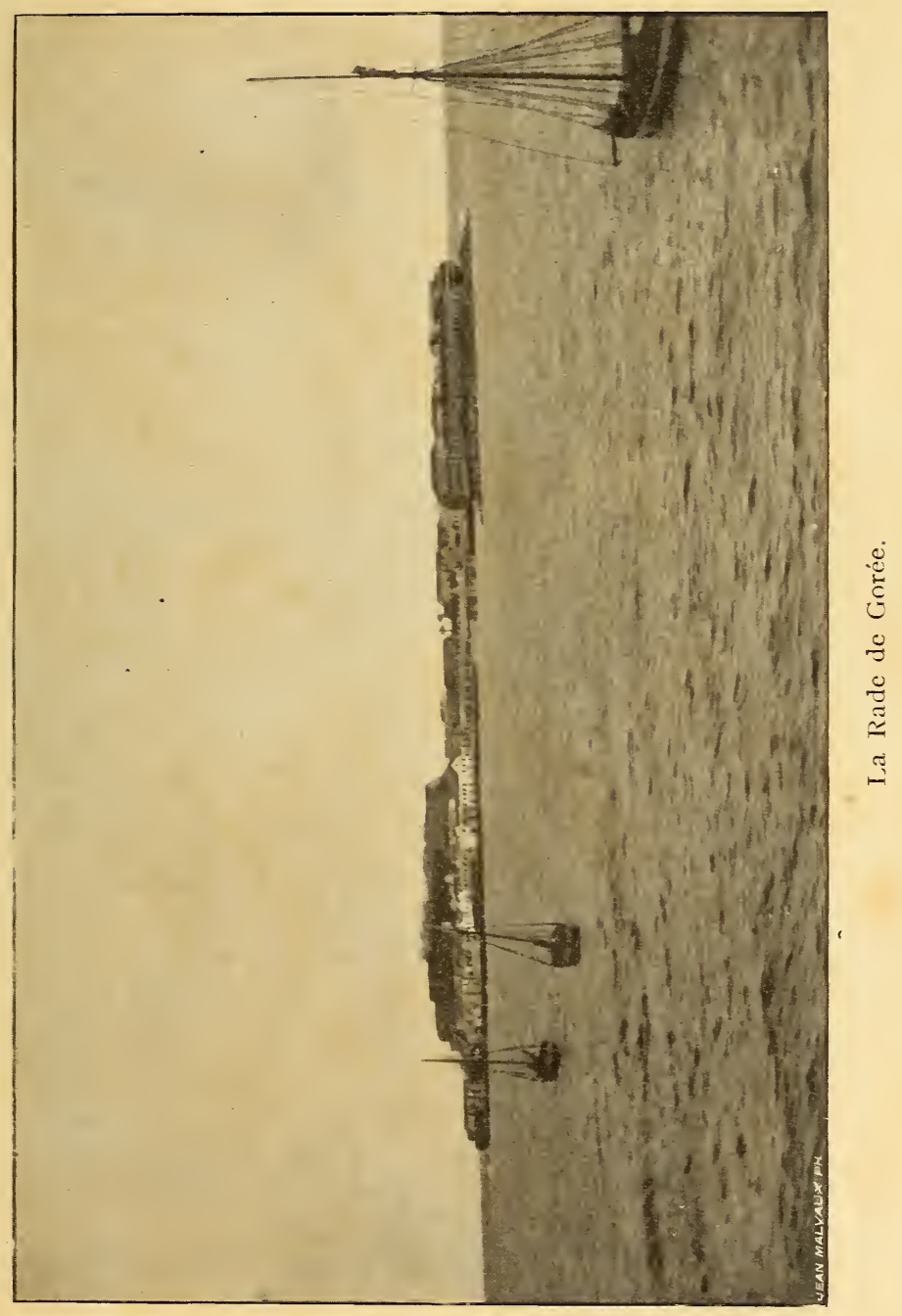




\section{I \\ LES ESCALES}

A bord de l'Albertillle, le 20 juin 1898 .

C'est à Lisbonne, le mercredi i 5 juin, que l'Albertville a fait relâche pour la première fois, depuis le départ d'Anvers. Dès le matin nous apercevons la terre qui arrête sur la masse des eaux ses contours indécis. A mesure qu'avance le navire se dessinent en leur harmonieuse ampleur les lignes de la baie. La rade étincelle au jour. Des oppositions de ton, d'ombre et de lumière vont des flots aux hauteurs en amphithéâtre dont les gradins à mi-côte sont occupés par d'innombrables édifices en rangées serrées. Immobiles spectateurs, dans l'éloignement, de leurs fenêtres clignotantes, ils ont l'air d'échanger des regards avec nous. C'est la 
ville. Elle est radieuse d'aspect; elle paraît immense. N'est-ce point la cité des marins héroïques, des fondateurs d'empires, des conquérants de l'Inde et de ses trésors? Décor prestigieux, mais décor surtout. De plus près l'intérêt s'atténue. Sans doute, la curiosité persiste des plans juxtaposés avec l'inattendu de leurs perspectives verticales. Mais les constructions ont une allure assez médiocre, dénotent une architecture plutôt banale. La monotonie rectiligne en est rompue seulement par les tons avivés des façades, où serpentent, de façon amusante, les arabesques bleues des revêtements de céramique.

La course rapide dans les rues ne laisse pas de donner quelque impression de délabrement. Si plusieurs monuments, l'arsenal, des portes, des colonnes surmontées de statues, évoquent les gloires du passé, celle de Vasco de Gama, aucune marque du présent n'accuse, nous ne dirons pas les sentiments d'orgueil et de puissance, si aisés à relever dans les grandes capitales, mais même ces caractères de santé, de confort, d'aisance épanouie qu'on retrouve partout dans notre petite Belgique. La physionomie générale de la population s'accorde, semble-t-il, avec ces dehors éteints. Depuis les quais, sans mouvement, malgré l'admirable disposition des lieux, jusqu'au centre de la ville, il passe comme un flottement de nonchalance. Au marché aux fleurs régnait de l'animation, mais point ou peu de cette exubérance méridionale à laquelle on s'attend.

Une excursion rapide à Cintra, la prestigieuse résidence royale, nous fournit l'occasion de voir la campagne de Lisbonne. La ligne du chemin de fer est bordée des 
deux côtés, aussi loin que porte la vue, par des champs de moissons maigres alternant avec le sol nu et rocheux, qu'ombragent à peine quelques eucalyptus. Les mouvements de terrain, très prononcés, avec le fond des montagnes finement découpées sur l'horizon, modèlent seules la matière plastique de la nature. A Cintra même le site se transforme comme par enchantement. A une demilieue de la gare, les voitures ont bientôt fait, au galop emporté de leurs attelages, de vous transporter dans un parc de verdure luxuriante où ruissellent l'eau et la fraîcheur. Des silhouettes fantastiques se profilent sur le ciel : rocs qui paraissent inaccessibles et où sont juchés manoirs et forteresses de la féodalité encore debouts et menaçants. Vision fugitive, car le temps presse; il faut retourner sur ses pas à la même vertigineuse allure.

$\mathrm{Au}$ départ de Lisbonne se place une courte cérémonie officielle. Le ministre de Belgique, M. du Bois d'Aissche, est venu saluer le colonel Thys. Les passagers vident tous une coupe de champagne. Souhaits de bon voyage, hommage ému au pays, tels sont les toasts dits ou pensés. En route!

Et le rêve panoramique du matin renaît dans les coulées d'or du jour chargé d'heures ensoleillées. La ville, par cela qu'elle s'étend le long de la rade, redonne la sensation de continuité qui la grandit si singulièrement. Le navire double lentement les points déjà fixés dans le souvenir à l'arrivée, depuis la tour de Belem jusqu'à la petite villa de Cascaes, à la pointe da Roca. Peu à peu les détails se perdent pour se noyer 
enfin dans la ligne d'horizon où se confondent l'eau et le ciel. L'Albertville a repris la haute mer; abandonnant le continent européen, il ne touchera plus désormais qu'aux terres d'Afrique.

Madère! tel est le cri qui vole de bouche en bouche, vendredi, vers Io heures du matin. Il ne s'agit encore que de l'îlot de Porto-Santo, qui fait d'ailleurs partie du groupe.

Soit dit en passant, il est curieux de noter combien ces petits points semés dans les profonds sillons de l'océan, à peine perceptibles sur les cartes de géographie, sacrifiés comme ils le sont aux gros pays, leurs frères, prennent de l'importance quand ils émergent soudain des flots. Terre! c'est la terre. Christophe Colomb, tout le premier, ne commença-t-il pas par une île relativement peu importante la découverte de l'Amérique? Les excursionnistes de l'Albertville découvrent aussi, à leur façon, ce qu'ils rencontrent en cours de route. Les notions géographiques leur sont servies en abondance, à supposer qu'ils ne les possèdent pas encore, par les livres et les cartes qui se trouvent à bord. A chacun d'y joindre ses impressions. L'image matérielle photographique, si l'on veut, puisqu'il n'est presque personne ici qui ne possède un appareil - est perçue à peu près de la même façon par tous les yeux. Mais elle vire et se développe dans les cerveaux selon mille modes. Ainsi s'enrichit sans cesse la littérature des voyages. Nul doute que celui de l'Albertville ne donne lieu à une production d'écriture incomparable, depuis les commen- 
taires de détail pittoresque qui relatent au jour le jour nos faits et gestes - gesta Dei per Belgas Congolensesques - jusqu'aux renseignements les plus authentiques sur l'Atlantide ou sur la production en vins, fruits et primeurs des îles Madère et Fortunées. Dans ce chœur de la presse il est permis à chaque exécutant de déchiffrer sa partie à sa façon. Le livre de la Nature est là tout grand ouvert : le Temps qui glisse rapide tourne les feuillets.

Après avoir doublé l'îlot de Porto-Santo et la petite ville de même nom blottie sous la masse chaotique de roches brunâtres, découpées comme à l'emporte-pièce, l'Albertville jette l'ancre, à I heure de l'après-midi, devant Funchal, la capitale, voluptueusement couchée au bord d'une baie en hémicycle. Abruptes, les pentes de la montagne s'élèvent à l'arrière-plan, recouvertes d'une riche végétation, jusqu'au moment où elles s'embrument de nuages. En couches superposées toute la gamme des tons est représentée, depuis le bleu prodigieusement intense de la rade jusqu'au fond de verdure sombre de la ligne d'horizon, en passant par les taches blanches, jaunes, rougeâtres des maisons. Ce paysage, de caractère à la fois doux et noble, s'est animé bientôt, à l'approche de notre bateau, du mouvement d'une foule de petites embarcations faisant force rames vers nous. Ce sont les colporteurs mercantis de l'endroit qui viennent offrir les produits de leur industrie, articles de vannerie, broderies, bijoux. Prestes et agiles, aidés d'une simple gaffe, ils réussissent à se hisser jusqu'aux bastingages. Ils s'agitent et crient : brouhaha pittoresque et 
encombrant. Les plus légèrement munis de ces arrivants ne sont pas les moins occupés. Le caleçon de bain est leur instrument de travail. Ils plongent comme des marsouins à la recherche des pièces de monnaie lancées du bord. Leur hardiesse est étourdissante. Tel d'entre eux est capable de se jeter à l'eau par tribord, du haut du pont et de reparaître à babord en passant sous la carène.

La descente à terre après une certaine attente s'opère vivement. Le rendez-vous général est la petite église de Notre-Dame del Monte, d'où l'on jouira d'un panorama merveilleux. Les groupes se divisent. Il en est qui choisissent la montée en traîneaux attelés de petits bœufs, équipage original s'il en fut, en son pittoresque de bucolique. Ce mode de locomotion demande un mot d'explication, en présence de l'antinomie apparente existant entre un traîneau et la surface pierreuse des rues. C'est que celle-ci est formée de caillous roulés, polis, rendus glissants à l'usage, mosaïque raboteuse d'aspect, mais parfaitement unie pour les patins de bois ou skys des véhicules qui ignorent les interstices sensibles seulement aux pieds des marcheurs. D'autres, dont nous sommes, ont préféré l'ascension plus rapide par le chemin de fer funiculaire. Quatre à cinq cents mètres en élévation séparent le fonds urbain du point où nous nous arrêterons. Cela suffit pour ménager à nos yeux émerveillés les transitions les plus riches, nous dirions bien les modulations infiniment variées de la flore de Madère. L'impression est ineffaçable de cette succession de jardins en paliers appuyés sur de forts murs de 
soutènement, où poussent fruits, fleurs, arbres de toutes essences.

Où trouverait-on, cohabitant en quelque sorte, le maïs, la canne à sucre, l'orge, le bananier, le citronnier, le figuier, la vigne? Les différences d'altitude font apparaître à faible distance les unes des autres les espèces tropicales et les espèces de nos climats, car chênes, sapins, pommiers et poiriers s'offrent aussi à nos regards. L'atmosphère également subit des modifications sensibles. Dans le bas, aux premières heures de l'après-midi, régnait une température lourde, chargée d'effluves de moiteur. Au point d'arrivée du funiculaire nous respirons plus librement. Par un chemin délicieux, ombragé d'un dôme de feuillée, nous accédons à la terrasse sur laquelle est édifiée la petite église. De là se découvre un horizon idéal. La mer, à nos pieds, scintille, tel un miroir, où se détache élégante et fine la silhouette de l'Albertville, notre bateau. Une brise heureuse nous enveloppe et nous pénètre. La vie est un rève...

La réalité reprend ses droits sous une forme amusante. C'est la descente en glissade dans un carossa, sorte de traîneau-boîte où prennent place deux passagers. Il dévale la pente vertigineuse, guidé par un conducteur, courant nu-pieds à vos côtés, une simple cordelle à la main, tirant de droite ou de gauche pour pivoter court aux tournants. Quelquefois l'homme pousse, le plus souvent il retient, sauf dans la ligne droite, où, juché à l'arrière, il se laisse aller à fond de train. Sans encombre, d'ailleurs, l'on arrive au bas de la montagne, dans cet automobile nouveau genre, à sensations fuyantes et, 
quoique la chose paraisse invraisemblable, nullement cahotées. La vue, pendant qu'on est en traîneau, est ordinairement limitée des deux côtés par les murs continus qui bordent le chemin ; le funiculaire, au contraire, domine le paysage. Les deux aspects se complètent, le second ayant l'avantage de nous rapprocher des habitants. Chose extraordinaire, et que nos cyclistes voudraient voir se généraliser en Belgique, des fleurs nous sont jetées au passage. Je puis donc noter que la race est d'humeur douce et accueillante.

En quelques heures nos impressions ou plutôt nos prises d'instantanés sont fixées. Il reste à parcourir la ville, toute solitaire, car c'est jour de fête et les maisons cèlent leurs habitants, assoupis sans doute dans une sieste bienfaisante. Le temps de faire quelques menues emplettes, de voir deux ou trois points curieux : la place du Gouvernement, où d'antiques figuiers étendent leur ramure multiple et drue, le jardin public avec sa flore tropicale, et de nouveau il faut partir.

$$
* * *
$$

A bord de l'Albertville, le rer juillet $\mathrm{I} 898$.

C'est droit sur l'Afrique que c'est dirigé l'Albertville à son départ de Madère, le I'j juin. Géographiquement l'île se rattache au continent noir; pour nous elle représente le dernier chaînon de la communauté européenne à laquelle nous appartenons, comme elle en est aussi 


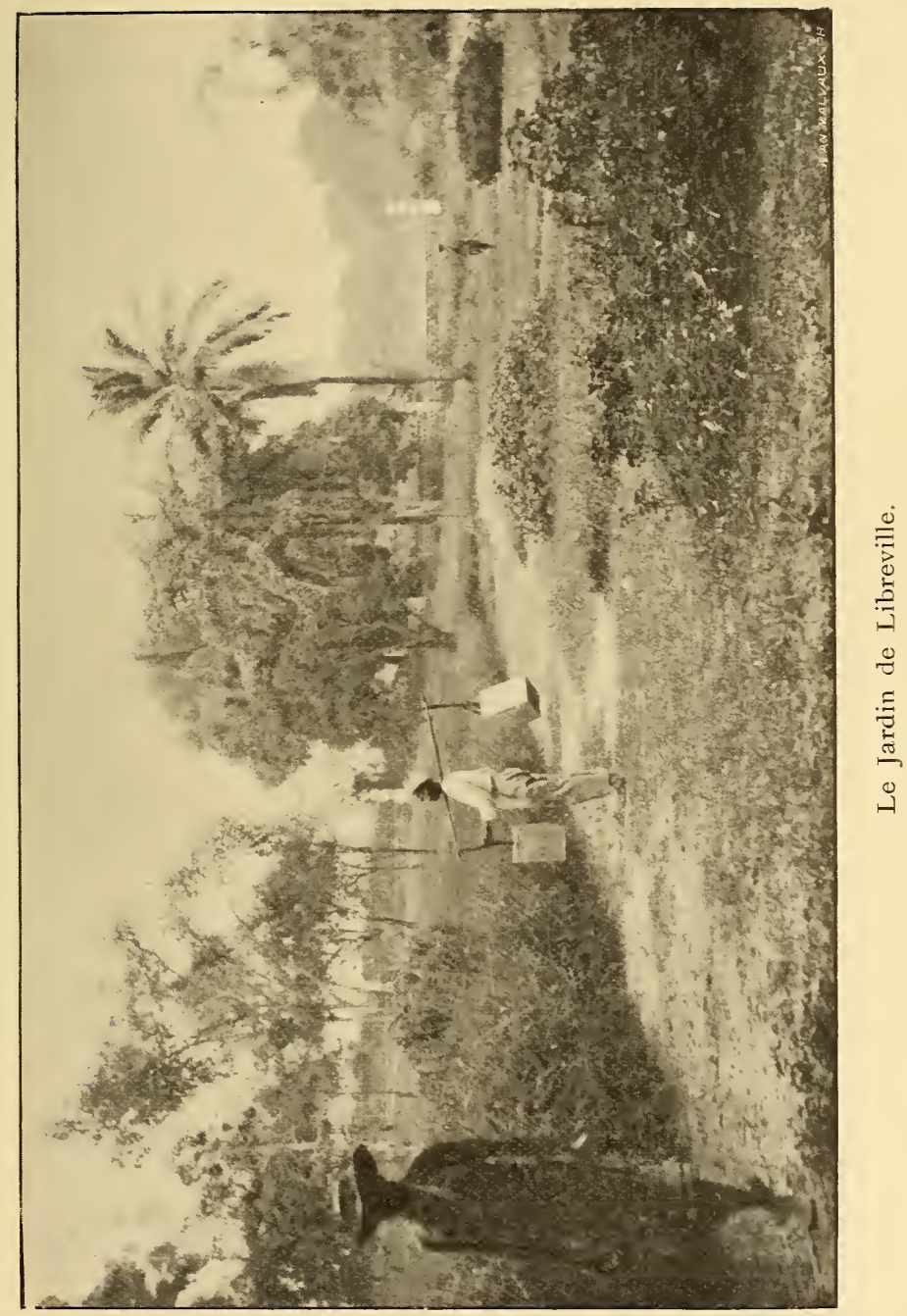



le sourire charmant et embaumé. Ce qui nous attire maintenant, c'est le mystère des choses à peine soupçonnées, ces pays où nous devenons les étrangers, les visages pâles. Notre curiosité se double de l'attente, cependant que lectures, impressions échangées, distractions variées du bord occupent nos plaisirs bercés d'un flottement doux et continu.

Le I8, la vue de Ténériffe est venue interrompre le calme paysage d'eau et de ciel. Tourmentée, hérissée de rocs sauvages, de lignes et de tons plus âpres qu'à Madère, la terre s'est dressée. Dominant les nuées, très net et distinct, le pic fameux si souvent caché, rapporte-t-on, aux regards des passants de la haute mer. Et l'impression est bien d'un séjour interdit aux mortels. Sur le mont, l'œil du rêve découvrirait peut-être quelque Walhalla, les dieux terribles ou bienfaisants et, chevauchant tout autour, la cohorte impétueuse des walkyries. Mais voici que les falaises brunes qui plongent dans les flots se sont évasées et entr'ouvertes.

A faible distance, Santa-Cruz, toute baignée de lumière, se découvre; la nature ici apaisée lui fait un cadre riant de verdure. C'est la réplique de tableaux déjà vus. Cependant, lorsque tombe le jour, la scène grandit démesurément de tout l'élargissement des lignes, de leur fusion dans l'infini. Le soleil jette sur l'horizon ses derniers feux, ses ors vieillis et ses pourpres, tandis que, dessinant de vagues et mouvantes figures, les contreforts de la montagne descendent dans l'onde et s'y perdent. Très haut, dans la sérénité du firmament, le cône, toujours visible. Tel un géant, il attire à lui, semble- 
t-il, les nuées épaisses pour s'en faire un manteau. Veille-t-il? ou dort-il?

Dans la nuit enfin venue, immense et bruissante, le bateau fuit, battant de l'hélice l'eau qui jaillit, laiteuse et bleue, avec des traînées de phosphore.

Le mardi 2 I juin l'Albertville est arrivé en vue du cap Vert. Nous apercevons, en premier lieu, deux mamelons assez haut placés, ayant forme de fortś, à la base desquels s'étend une bande de sable jaune rougeâtre. La végétation est clairsemée : de-ci de-là quelques arbres à l'aspect décharné. Nous approchons de Dakar; des maisons couvertes de tuiles rouges tranchent sur le bleu implacable du ciel. Il est midi; le soleil au zénith darde de brûlants rayons. Mais la brise est fraîche; rien donc ne s'oppose à une brève visite de la ville. Avant cela, les préliminaires de l'opération de débarquement durent assez longtemps pour rendre aisée l'orientation première des yeux et des idées.

La rade en elle-même se présente sous un aspect fort séduisant. Vers le large elle est partiellement barrée par le petit îlot de Gorée, occupé jusque dans ses moindres recoins par de pittoresques constructions qui, en épousant les aspérités du roc, lui donnent un air de bijou à facettes, scintillant sous la chaude caresse du soleil. Sur la terre ferme, moins curieuse, mais plus considérable à coup sûr, est installée Dakar. Elle a des ambitions et 
elle ne faillira pas à les justifier. Si elle ne possède pas encore les aménagements d'un vrai port, elle se prépare, par l'amorce de quelques travaux, à l'œuvre de transformation plus ou moins prochaine. La superbe nappe d'eau comprise entre Dakar et Gorée et les promontoires de la baie se prêtent à abriter des flottes entières.

Le long de cette côte inhospitalière les accès faciles sont rares. Celui-ci était une invite naturelle à l'établissement des Européens. L'on sait, par ailleurs, que l'importance grandissante prise par le Sénégal et ses annexes du Soudan dans le domaine colonial de la France confère à Dakar les caractères d'une base politique et commerciale de premier ordre. Saint-Louis, la capitale, est exposée aux graves inconvénients de la - barre à l'embouchure de son fleuve. Son vrai port, c'est précisément Dakar, à laquelle elle est reliée par un chemin de fer depuis quelque douze ou treize ans. Tant à la sortie qu'à l'entrée Dakar emporte la plus grosse part des transactions de la colonie qui se chiffrent annuellement par environ 40 à 50 millions de francs, Saint-Louis conservant sa prééminence de grand entrepôt du marché de l'intérieur, tête de ligne de la navigation fluviale.

En dehors de sa position privilégiée, dont la signification ne saurait échapper à personne, Dakar présentait pour nous le mérite spécial d'établir un premier contact avec les indigènes. Cela ne tarda guère. Les plus empressés à venir ou à nager vers nous furent les virtuoses du plongeon qui recommencèrent les prouesses admirées chez leurs émules de Madère. Seule la couleur de la peau était différente. 
Plus neuve et plus intéressante fut la descente à terre. Du centre de la rade, où l'Albertville est ancré, à la petite jetée, ce sont des barques à voile qui se chargent du service de va-et-vient. Dans celle où nous avons pris place, un superbe Toucouleur commande et dirige la manœuvre qu'exécutent plusieurs noirs. Limpression n'est nullement défavorable. Le patron, avec sa mine fière, son boubou flottant bleu et blanc, la stature haute et élancée, eût passé partout pour un bel homme. Les hommes de l'équipage, de traits plus grossiers, n'avaient nullement une face inintelligente. Des Ouolof, nous dit-on. Et c'est en effet le type dominant dans cette partie du Sénégal. Grands et forts, ils alimentent la colonie en soldats et en travailleurs dont le rendement est fort prisé. Nous en savons quelque chose au Congo, où, de l'avis unanime, ils ont été les meilleurs ouvriers du chemin de fer. Ils ont "un jet de pelle de 6 mètres " aux terrassements, nous dit d'eux un ancien Congolais. Et dans cette forme concrète, la louange, on le reconnaîtra, a son prix.

En une demi-heure, nous abordons un petit môle qui, avec une jetée courant dans le sens transversal, forme un bassin rectangulaire, encore en voie de construction.

Des groupes bariolés se tiennent sur le quai, hommes et femmes. Celles-ci, drapées assez gracieusement dans des pièces d'étoffes à couleurs voyantes, des foulards noués en forme de turbans sur la tête, la démarche balancée, ne manquent point de cachet. Les cheveux, disposés en petites tresses serrées, encadrent un visage qui chez plusieurs a du galbe. Le hasard de la prome- 
nade nous mène au marché où sont rassemblées de nombreuses vendeuses de poissons. Et les appareils photographiques de fonctionner. Mais aussitôt ce sont des cris, des débandades folles, où la joie se mêle à une sorte de peur du fétiche, du mauvais sort lancé par l'objectif. N'importe! bien des attitudes ont été surprises à temps; bébés juchés sur le dos ou sur le flanc de leurs mères et sœurs, retenus par le boubou qui, serré dans le bas de la taille des porteuses, devient la sacoche d'où émerge une petite boule frisée, éclairée par d'énormes yeux; plus loin, une femme qui, sur la tête, d'un bras arrondi selon le dessin le plus classique, retient une charge ou la cruche d'eau puisée à la fontaine. De la gaîté, du babillement, de l'animation en des gestes cependant nonchalants, tout cela constitue pour nous un tableau plein de saveur.

Des maisons, un marché, une église, une caserne, des rues, voilà pour le quartier européen. Il souffle, ou il a soufflé ici, à n'en pas douter, un air embrasé. Les arbres, tout écorce, qui bordent les voies tracées à angles droits, secouent plus de poussière qu'ils ne répandent d'ombre. Allons un peu plus loin, nous en avons encore le temps.

Que deviennent les indigènes dans ce milieu? Ils ont leur faubourg à part, traversé d'allées de terre fortement tassée. Autant de familles, autant de petits enclos, semble-t-il, les séparant les unes des autres. En arrière de la palissade à claire-voie, à mi-hauteur d'homme, est érigée la cabane, simple rez-de-chaussée en bois, en torchis, mais généralement recouverte de tuiles, qui piquent des notes gaies et claires parmi les paillotes voisines à 
l'aspect morose. Tantôt accoudés aux chambranles des portes d'entrée, tantôt accroupis au pas de celles-ci, ou circulant par petits groupes, les indigènes regardent passer, assez indifférents, la caravane casquée de blanc, les excursionnistes aux yeux avides. Pour quelques menus achats, il est possible de palabrer avec ceux d'entre eux qui entendent et baragouinent le français. Après une heure de marche, nous retournons fidèlement à l'Albertville, la vision enrichie de quantité d'images. Voyager et voir n'est-ce pas vivre, au meilleur sens du mot?

Entre Dakar et Libreville une escale imprévue est venue s'ajouter à celles qu'avait fait connaître le programme du voyage. Celui-ci comportait l'arrivée dans l'estuaire du Gabon, le mardi 28 juin, avant la nuit. Or, dès la veille la chose n'était plus possible. Alors qu'une légère perte de temps sur les prévisions s'était déjà produite, l'Albertville fut arrêté dans sa course par un événement aussi triste que fortuit. Lundi matin, un homme de l'équipage, occupé à un travail de nettoyage à l'extérieur de la carène, tomba à l'eau. Des secours furent aussitôt organisés, les bouées lancées par-dessus bord, une embarcation mise à l'eau. Le navire stoppa, puis décrivit un cercle complet pour regagner dans son sillage le point approximatif où l'on avait vu choir le matelot et où, affirmait-on, il se maintenait à la surface en nageant ferme. Vaines recherches! L'abîme avait saisi sa proie pour ne plus la lâcher. La route en avant fut reprise. Équipage et passagers, tous, l'angoisse au cœur, nous devions nous rendre à l'inévitable : La mort, si lointaine et si proche... 
L'Albertville jeta donc l'ancre devant San-Thomé le mardi matin. L'île figure en bon rang parmi les plus beaux établissements coloniaux qui soient. Nous n'avions évidemment pas le loisir d'aller admirer sur place les cultures si remarquables qu'y ont entreprises les Portugais. Du large, l'opposition est entière avec le paysage desséché de Dakar. Dans la buée du matin, San-Thomé ressemblait pour ainsi dire à un paysage de petite Suisse. Le sol est verdoyant, revêt l'aspect d'un grand parc. Quelques moments passés à terre, après plusieurs heures de bordées invraisemblables dans un boutre indigène, nous permettent de contempler les premiers cocotiers, - pour nous, - quelques superbes échantillons de la flore équatoriale. Sur le quai, des sacs de café et de cacao mis en piles témoignent de la prospérité de l'importante industrie agricole pratiquée dans lîle.

Libreville, où nous avons touché le lendemain, le mercredi 29 juin, nous a familiarisés de plus près avec la végétation de cette partie de l'Afrique. L'intérêt de cette station, son titre à l'admiration aussi, c'est son superbe jardin. Attenant immédiatement à l'habitation du gouvernement, il s'étend sur plusieurs hectares de terre que l'on nous dit ne pas être cependant de qualité exceptionnelle. Il faudrait tout un catalogue pour énumérer les diverses espèces sélectionnées ou acclimatées ici. Les différentes sortes de caoutchouc, le cacaoyer, le poivrier, le colza, le cotonnier, bien d'autres essences, dont les plantations sont en voie de réussite, sont représentées dans cette flore semi-naturelle, semi-artificielle.

Dans l'estime du directeur des cultures qui nous con- 
duisait, ces beaux résultats botaniques l'emportaient probablement de beaucoup sur le côté pittoresque. Nous ne songions d'ailleurs point à les déprécier en nous laissant aller au charme presque enivrant d'une promenade exotique sous le dôme des raphias ou des manguiers, en nous arrêtant ébahis devant un gigantesque fromager, l'inextricable fouillis de hauts bambous, les dattiers si droits et si élancés, les cocotiers au port élégant et souple. Des travailleurs assez nombreux sont employés à l'entretien des jardins. C'est ainsi qu'il nous a été donné d'apercevoir quelques types indigènes. A part de rares exceptions, ils s'écartent sensiblement du modèle esthétique que nous portons en notre for intérieur, - voire du type évidemment supérieur rencontré au Sénégal.

La halte de Libreville avait pour objet immédiat d'amener à bord de l'Albertville, parmi les invités officiels de la Compagnie du chemin de fer, M. de Lamothe, le commissaire général du Congo français. A Cabinda, de même, le gouverneur portugais sera pris au passage, demain $\mathrm{I}^{\mathrm{er}}$ juillet. C'est notre dernière étape avant le Congo, où nous parviendrons le même jour, l'attention appuyée en quelque sorte sur ces points de repère déjà sûrs, ces belles escales, dont fut comme jalonnée notre heureuse traversée. 


$$
1
$$





\section{L’ARRIVÉE}

Vendredi, I er juillet, l'Albertville est entré dans l'estuaire du Congo. Depuis plusieurs heures déjà, les vagues glauques de la mer se sont colorées de traînées limoneuses; elles se sont affaissées dans le clapotis du courant, devenu maître de la houle. De la masse puissante du fleuve étalé se dégage aussitôt une impression de grandeur et de majesté. Le cadre est bas sur l'horizon, de simples linéaments teintés de vert ou de bleu. L'ébauche est à peine tracée et pourtant que de choses elle exprime. C'est la première phase du voyage accomplie, le but touché, terre promise où nous retrouverons, autant que nous les découvrons, sol, hommes, pavillon 
qui sont nôtres. Le Congo, pays étranger, eh oui! mais, au-dessus de la fiction des mots, le Congo belge. La réalité s'impose ainsi, singulièrement émouvante, après les escales exotiques qui nous prenaient surtout par la curiosité, ce sixième sens en lequel se cristallisent tout d'abord nos impressions physiques.

Insensiblement les formes s'affermissent. Les cartes déployées peuvent nommer les contours visibles à présent. A notre gauche, la pointe française, Moanda, où est érigée une mission, avec des bandes de verdure et des plaques jaunes de terrain dénudé alternant les unes avec les autres. A notre droite, la pointe portugaise dite aussi " des requins ", plage de sable derrière laquelle s'élève un écran de végétation. Sous un ciel voilé, quoique de lumière blanche assez intense, tout cela émerge lentement des fonds vaporeux. Les arbres prennent figure; il en est qui sont de frondaison chenue, tandis qu'à côté d'autres - les baobabs ventrus - étendent tristement leur ramure comme dépenaillée. Les collines vertes semblent reculer dans le lointain; à l'avant-plan ressortent nettement les points blancs qui, du large, semblaient les moucheter. Voici Banana, édifiée sur une étroite et assez longue pointe de terre qui s'étend entre la mer et une sorte de crique intérieure du Congo.

Un incident imprévu nous a permis de reconnaître de plus près que ne le laissait supposer l'itinéraire de l'Albertville, ce poste avancé de la terre congolaise. L'étiage des eaux du fleuve est tel en ce moment que le bateau, pour le remonter, doit au préalable s'alléger. Le pilote et capitaine du port l'a ainsi décidé. Un autre 
vapeur, le Coomassie, également ancré devant Banana, se trouve 'dans le même cas que l'Albertville. Le fait paraît bizarre, quand on considère le débit énorme des eaux du Congo qui certes auraient su lui créer un lit suffisamment profond. Mais il y a les bancs de sable, de régime variable, à travers lesquels les passes sont plus ou moins rares et d'accès malaisé aux navires d'un certain tonnage. La nuit sera donc employée à délester l'Albertville, dans la mesure nécessaire, d'une partie de son charbon. Contretemps qui n'en est pas un, puisqu'il rend possible une promenade à terre.

Banana est fort déchue de sa situation passée, depuis que le mouvement maritime, suivant l'œuvre de pénétration vers l'intérieur, s'est reporté bien en amont du fleuve. Cependant on y trouve encore plusieurs factoreries, hollandaise, portugaise, belge. Quelques pépinières marquent également l'installation de l'État. Palétuviers et cocotiers, de belle venue d'ailleurs, forment le fond de la végétation. Des habitations sont disséminées de-ci de-là. Chez les résidents européens, elles réalisent le type que nous rencontrerons souvent désormais : toits blanchis surplombant la véranda circulaire; enclos très coquettement tenus. Chez les indigènes, des cases ou paillotes, d'aspect sale ou miséreux. Tout ceci n'aurait pas retenu outre mesure notre attention, n'eût été la nouveauté du décor et, en cette soirée inoubliable, un jeu magique de lumière qui eut bientôt fait de transformer en scène de féerie ce coin perdu de la côte africaine. Par l'étroite sente de sable ou de terre battue, bordée d'herbes rousses, la promenade se poursuivait 
pittoresque. Du côté de la pointe où la mer apporte à la grève ses ondulations lentes et molles, soudain le soleil, embrasant le ciel et les flots de lueurs sanglantes, descendit, œil énorme et rouge dont la vague était la paupière. De l'autre côté, sur l'eau dormante de la crique, se leva alors la lune. Et ce disque pâle, qui rapidement montait au zénith, éclairait de façon singulière. Ses rayons indécis semblaient faire la neige sur les toits blancs, en laissant d'impénétrables cônes d'ombre dans l'épaisseur des taillis; en même temps ils réfléchissaient intérieurement sur nos âmes.

Lumière peuplée de songes. Du sol, des végétations étranges, des mares croupissantes, de la nuit silencieuse que rythment seuls, de leurs battements continus d'ailes, les grillons', s'exhale une sorte de souffle mystérieux. Aucun malaise physique. L'atmosphère est calme, la brise tiède, la nature repose silencieusement alanguie. Mais l'essor est donné aux pensées qui vont là-bas, d'où l'on vient, doucement, tristement...

Ce soir même, à bord de l'Albertville, le cœur se reprend aux contrastes réconfortants. Le I er juillet a ramené l'anniversaire, le treizième, de la fondation définitive de l'État Indépendant, de la proclamation de la souveraineté du roi Léopold. Le colonel Thys a tenu à rappeler à ses invités cette mémorable circonstance. A l'issue du dîner il a prononcé un toast éloquent, qui a été salué des vibrantes acclamations de l'assistance. Hurrah! hurrah! trois fois hurrah! avons-nous crié, et avec nous, les étrangers, dont S. Exc. M. le conseiller Kologrivoff s'est fait le chaleureux interprète. En de tels moments, 
l'on comprend le prix des élans du patriotisme. Les hommes d'une même race, d'un même pays s'entendent, les cœurs se gonflent d'une émotion saine et forte. $\mathrm{Au}$ Congo, dans la colonie, revit la Belgique.

\section{$* *$}

L'étape d'une journée que comporte pour les navires venant de la haute mer la route fluviale du Bas-Congo, jusqu'au point où s'arrête la navigation, montre déjà la contrée sous des aspects multiples.

Vers l'embouchure, la nature s'est montrée prodigue. Des rideaux de forêts, à la trame serrée, arrêtent la vue, à droite comme à gauche; sur la rive sud, la portugaise, que nous longeons de près, l'œil peut d'ailleurs suivre les caprices de cette flore exubérante. Le sous-bois surtout marque la différence d'avec les paysages sylvestres des latitudes tempérées. A côté, pour ainsi dire, de la futaie, d'où émergent, droits comme des I, les cocotiers, les élaïs, ou encore, véritables géants, les faux cotonniers, se développe une végétation prodigieuse. La brousse se fait taillis, la plante devient arbre, la liane joint la cime à la racine, unit entre eux ces piliers innombrables, tous ces troncs puissants ou sveltes et l'ensemble forme un fouillis d'apparence inextricable, tamisant, divisant à l'infini la lumière du jour. Par instants des échancrures ouvrent la rive, expansions ou criques du fleuve, et courts affluents, au-dessus desquels les ramures se rejoignent 
presque en dômes mouvants, assombrissant l'eau qui les reflète.

Plus loin, les levées de terre, qui faiblement dominent le fleuve, se dénudent ; au milieu du courant surgissent des îles basses, arrosées et fertilisées à souhait. Pays de pâturages à l'herbe grasse; c'est ici qu'est située Mateba, où prospère un nombreux bétail élevé par les soins de l'homme blanc.

Ailleurs encore ce sont de grandes échappées panoramiques, effets de ciel et d'eau, tant les lignes de terrain ferme sont abaissées dans la largeur démesurée du Congo. La sylve ayant disparu, la brousse a pris sa place, ou le roc, tandis que les monts de Cristal, au loin, semblent barrer l'horizon. La roche fétiche, telle une sentinelle postée sur la route des navires, masque seule encore la vue de Boma. Passé le promontoire, la capitale apparaît enfin dans le flamboiement de l'heure de midi.

Ses abords, en aval, sont gardés, à flanc de coteau, par les batteries de Shinkakasa, qui ont donné de leur grosse voix, à l'approche de l'Albertville, en signe d'allègre bienvenue; et la "rivière des Crocodiles ", du côté de l'ouest aussi, lui fait, à moindre distance, un fossé passablement sinistre. Aux factoreries isolées aperçues jusqu'ici, sans parler des chimbèques des noirs, sortes de termitières disséminées dans la brousse, a succédé une véritable agglomération, dont la physionomie "ville " s'accuse aussitôt. La rive, comme il est naturel, a été occupée en premier lieu. La cité naissante a gravi ensuite la colline à laquelle elle était adossée. 


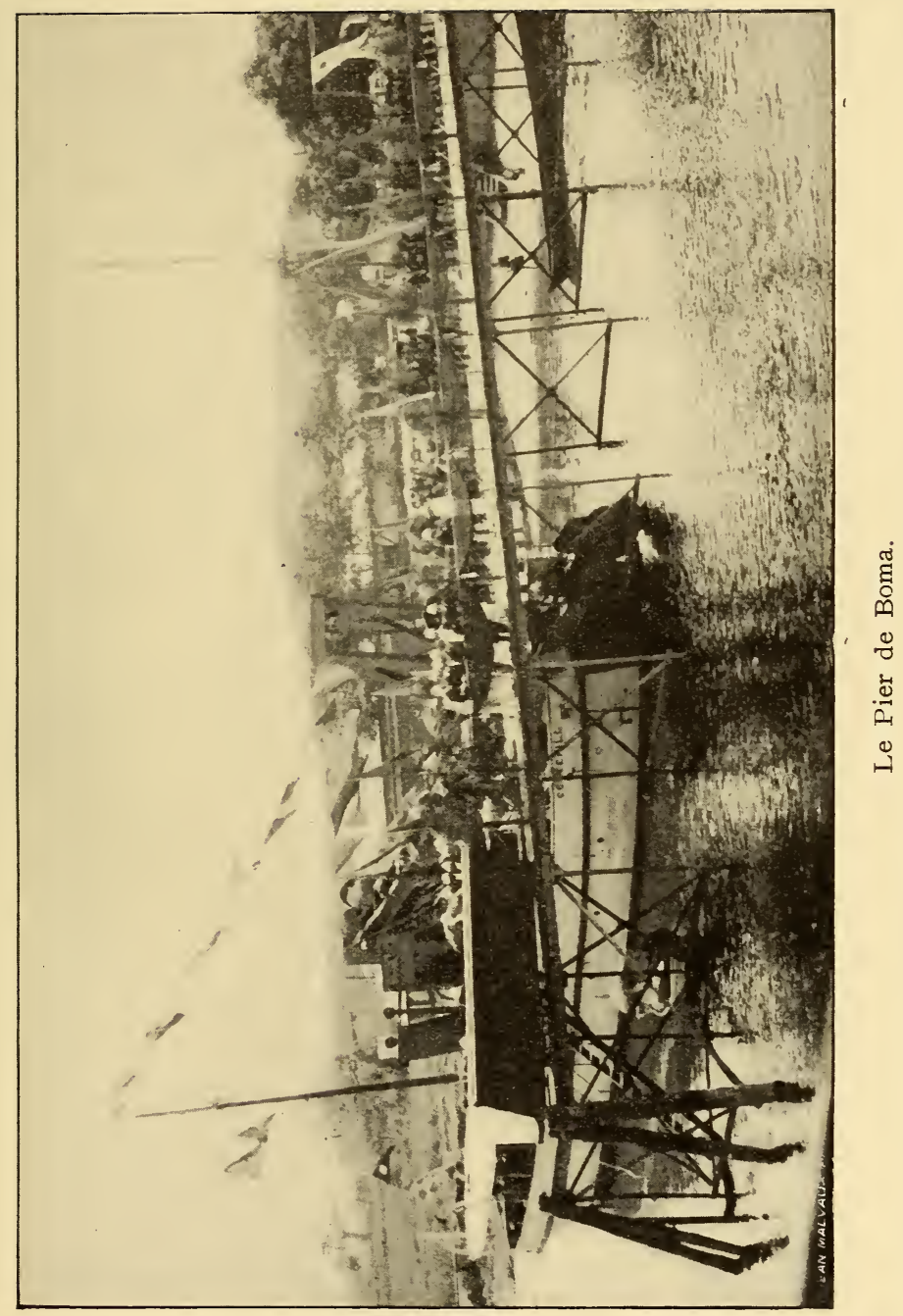



Sur le plateau ont été élevés les principaux édifices, avec l'église en fer qui les domine de son clocher. Les plans sont ainsi suffisamment étagés et étendus pour ménager d'agréables perspectives et créer en outre l'impression d'un centre sainement constitué.

Voilà pour le décor extérieur de la réception du 2 juillet, dont, en ce moment, nous ne pressentions guère le caractère aussi imposant qu'émouvant. Le retard subi en cours de route n'a fait que remettre d'un jour le programme de la fête nationale. Nous sommes attendus. Et l'on nous fait belle conduite. Le pier d'accostage est décoré de pavillons multicolores que la brise agite gaiement. A son extrémité sont groupées les autorités, M. Fuchs, gouverneur général par intérim, en tête. A bord, dignitaires, officiers, fonctionnaires, simples particuliers ont revêtu l'uniforme ou l'habit de cérémonie. Le cortège s'ébranle, tandis que la compagnie d'honneur sonne aux champs et que s'exécutent brefs et fermes les commandements militaires. La Brabançonne retentit pendant que le général Daelman passe en revue la troupe admirablement alignée, les hommes immobiles sous les armes comme autant de statues de bronze. Les jeunes soldats de la colonie scolaire, les enfants de troupe de là-bas, n'ont pas moins bonne mine et leur musique est particulièrement bien rythmée. Des deux côtés de la large avenue qui s'amorce sur le pier par une courte allée de beaux cocotiers, sont massés les noirs, hommes et femmes, aux pagnes bariolés, les visages curieux et éveillés, tout à l'intérêt du spectacle que nous leur donnons. 
Sans perdre de temps nous montons dans le tramway à,vapeur en destination du plateau. Le trajet est vite parcouru dans la fuite rapide des maisons ou des plantations de bananiers qui bordent cette rampe en pente douce. Arrivés là-haut, nous mettons pied à terre pour gagner par de belles avenues soigneusement tracées et entretenues la petite esplanade où est érigée l'église. Maint village de notre pays en possédera peut-être une d'aspect plus monumental. Celle-ci cependant, à l'heure où tintent plutôt grêles les appels tombés de son clocheton, s'élargit en quelque sorte des proportions que lui confèrent le lieu et les circonstances. Sous la modeste voûte de tôle, dont l'ingénieux agencement ne dissimule pas le caractère primitif, dans l'appareil d'une pompe religieuse réduite à des moyens d'expression presque naïs, se célèbre un service religieux d'ordre infiniment élevé. Au pied de l'autel de chêne sculpté, orné de drapeaux et de palmes, devant une assistance venue de loin, le prêtre appelle les bénédictions du Très-Haut sur le Souverain du vaste empire fondé en Afrique, au cœur de la barbarie. Un symbole puissant plane ainsi au-dessus des représentations imparfaites : statues coloriées, chemin de la croix d'inspiration rustique. Du dehors, le canon tonne, scandant, à intervalles réguliers, les chants liturgiques auxquels prennent part, avec des voix qui résonnent bizarrement en latin, les jeunes noirs placés au jubé, guidés et soutenus par les harmonies graves de l'orgue.

Après le Te Deum eut lieu le défilé des troupes devant le général Daelman placé, ainsi que sa suite, sur 
la terrasse del'ancienne habitation du gouverneur. Quatre compagnies passèrent ainsi, étonnamment correctes, martiales et fières d'attitude. Elles étaient conduites au pas de parade par les officiers et sous-officiers blancs, leurs chefs, leurs éducateurs, leurs supérieurs, exécutant le salut des épées nues devant le front des baïonnettes étincelantes. Quel beau moment! De la nuque aux talons un frisson vous fait tressaillir. La réception officielle est close. Elle a duré en tout deux heures, pendant lesquelles la rapidité des impressions a contribué de fait à en augmenter l'intensité. Toutes se condensent en une notion dominante d'ordre, de discipline, d'effort conscient. La première ouverture ménagée au seuil laisse ainsi voir les murs maîtres qui soutiennent l'édifice de l'État. Assis sur bon sol, ils sont établis selon les lois d'un juste équilibre. Il y a ici des idées-forces.

Désormais la pensée souveraine d'organisation s'impose, irrésistible, à qui veut la saisir. Elle a vivifié le corps entier dans lequel elle s'est implantée. Les résultats sont là qui s'annoncent : les grands travaux, les cultures, les progrès économiques, le développement de la civilisation.

Dans la même journée du 2 juillet nous arrivons à Matadi, le second battant - Boma étant le premier de la large porte placée sur le bas fleuve. Il ne faut pas longtemps pour se rendre compte de la nature des obstacles qui, derrière celle-ci, viennent se dresser. Après plusieurs rappels, superbes du reste, sur l'une ou l'autre rive, des végétations richement épanouies, telle la luxuriante île des Princes, le Congo s'est définitivement 
assombri. La berge ressemble à un glacis de redoute; hargneuses, les montagnes accroupies étranglent la masse d'eau qui furieusement se livre passage. Voici le " chaudron d'enfer " et enfin, à la sortie de la courbe tourmentée où les flots tourbillonnent, la ville, point de départ du chemin de fer.

Matadi nous réserve un accueil non moins triomphal que celui de Boma, avec une nuance de spontanéité communicative en plus. Les hurrahs éclatent vibrants, avant même que nous soyons amarrés à quai. Juchés sur les contreforts qui immédiatement montent du fleuve et qui sont entaillés par les routes et plates-formes pratiquées par les ingénieurs, tous les bâtiments sont pavoisés. Le coup d'œil est extrêmement pittoresque, à la tombée du jour. Dans la lumière crue du soleil, le caractère faubourg de cité manufacturière se marque davantage. Telle quelle, c'est une vraie ville-champignon qui a poussé là, devançant la mesure ordinaire du temps. La rive opposée, en tous points semblable à celle où nous nous trouvons - d'après ce qu'on nous rapporte - avant la fondation de Matadi, témoigne, en son abrupte désolation, du travail accompli, et au prix de quels efforts, on le devine sans peine.

A l'heure actuelle, tout est à la joie et aux réjouissances. Le soir, des députations d'ouvriers noirs sont venues au pier d'accostage donner, en l'honneur des. passagers de l'Albertville, des sérénades de leur goût. Divisés par groupes, des gens de Sierra-Leone, d'Accra, de Lagos, porteurs de drapeaux, de pancartes, de torches et de lanternes vénitiennes, se sont livrés à des. 
exercices variés de chant et de danse, spectacle de débraillé curieux et drolatique.

Bientôt plus rien ne manque à la fête, ni les lampions dont les cordons lumineux sillonnent les maisons, ni même le feu d'artifice aux pétarades divertissantes, aux fusées, aux gerbes qui, éblouissantes, raient cette nuit équatoriale qu'il fautun effort singulier de l'imagination pour se figurer étendue sur une contrée si lointaine et, le croirait-on, si mauvaise.

Le séjour à Matadi comprenait le repos du dimanche 3 juillet, ce qu'il fallait pour une courte reconnaissance des lieux. A 9 heures du matin, une messe fut célébrée qui emprunta au cérémonial du Te Deum de Boma l'appareil militaire et les coups de canon. Elle en différa par l'allocution qu'adressa le père D'Hooge à l'assistance. Dans un langage libre et tout de circonstance, l'orateur exalta la grande pensée du règne du roi Léopold, l'œuvre du chemin de fer, les mérites de l'illustre colonel Thys.

Quelques heures de flânerie nous firent assister aux scènes inédites d'un petit marché indigène et nous menèrent ensuite dans les différentes factoreries fondées par nos compatriotes. Ceux-ci n'ont point méconnu l'importance intrinsèque de la place, destinée, qui sait? à devenir la métropole commerciale du Congo, où afflueront les produits de l'intérieur et les articles d'échange de l'Europe. De là une concurrence déjà très accusée entre commerçants. A côté des Magasins généraux, arrivés bons premiers, il y a la Belgica, la Congolia, d'autres encore. C'est le genre bazar d'utilité qui a été adopté dans ces maisons. On y trouve denrées 
et conserves alimentaires, vêtements et équipements d'Afrique, articles de ménage. Bien entendu ce commerce de détail n'est pas exclusif d'entreprises plus considérables; tel quel, il est rémunérateur, à raison de l'affluence croissante des blancs aux mille besoins desquels il faut pourvoir.

A une agglomération qui d'ores et déjà a pris cette importance, il faut un centre de réunion accordant une certaine place aux choses de l'esprit. Aussi Matadi possède-t-elle une bibliothèque à la création et à l'entretien de laquelle l'État et surtout la Compagnie du chemin de fer ont prêté le plus bienveillant concours. Placée sous la direction des pères D'Hooge et Janssen, cataloguée sur le modèle de la classification décimale, elle n'est formée que par des contributions gracieuses dues aux donateurs de la mère patrie principalement. Ceux-ci sont assez nombreux pour pouvoir mettre à la disposition des lecteurs un total vraiment considérable de journaux, de revues, de magazines publiés en différentes langues, le tout au courant à un léger retard près. Des romans, des livres de science vulgarisée sont également rangés sur les rayons. Soit dit en passant, l'œuvre mérite d'être sincèrement encouragée et soutenue.

Pour compléter la physionomie de Matadi, il convenait de prendre une vue à vol d'oiseau des environs. L'occasion toute trouvée était une visite à la mission de Kikanda, située sur la hauteur, à une heure de marche environ. A mesure qu'on s'élève, se découvre le chaos des larges crêtes dénudées qui chevauchent les unes sur les autres en files interminables. L'altitude de ces 
croupes pourrait les rapprocher de certaines parties de nos Ardennes, mais en cette saison de l'année elles sont brûlées, roussies, pelées, pierreuses, réduites à l'état de squelettes massifs et monstrueux, sauf dans les endroits où court un filet d'eau qui, de suite, fait surgir le fourré ou l'arbre.

$\mathrm{Au}$ milieu de cette solitude, l'hôpital de Kikanda représente bien le refuge idéal, le secours espéré, le repos goûté en son entière douceur. Un père et quatre sœurs sont les occupants actifs et dévoués de cette oasis, établie à proximité d'une source. Maisons d'habitation, hôpital, dépendances, petite ferme avec jardin potager forment une sorte de quadrilatère. Quand on y pénètre, tout respire le confort, les soins délicats, presque la coquetterie. Quelles sont les mains qui vaquent à tant de besognes minutieusement accomplies ou surveillées? L'attente n'est pas longue. Voici que s'avancent la mère supérieure, déjà plus âgée et plus émaciée, aux traits empreints de gravité souriante, et les autres, dont cette petite sœur aux yeux bleus, si doux, si confiants et tranquilles. Sur le visage de chacune d'elles est peinte la joie intérieure, fleur rare, à l'arôme subtil, qu'effleure seule l'aile des anges. Et la vision touchante nous suit sur le chemin du retour, quand nous reprenons à travers le dédale des côtes poussiéreuses que le soleil à son déclin rend vermeilles.

La conjonction des astres nous est-elle propice? La nuit venue, une éclipse de lune en son plein s'offre admirablement nette et distincte à nos yeux presque las de tant de spectacles somptueux. 



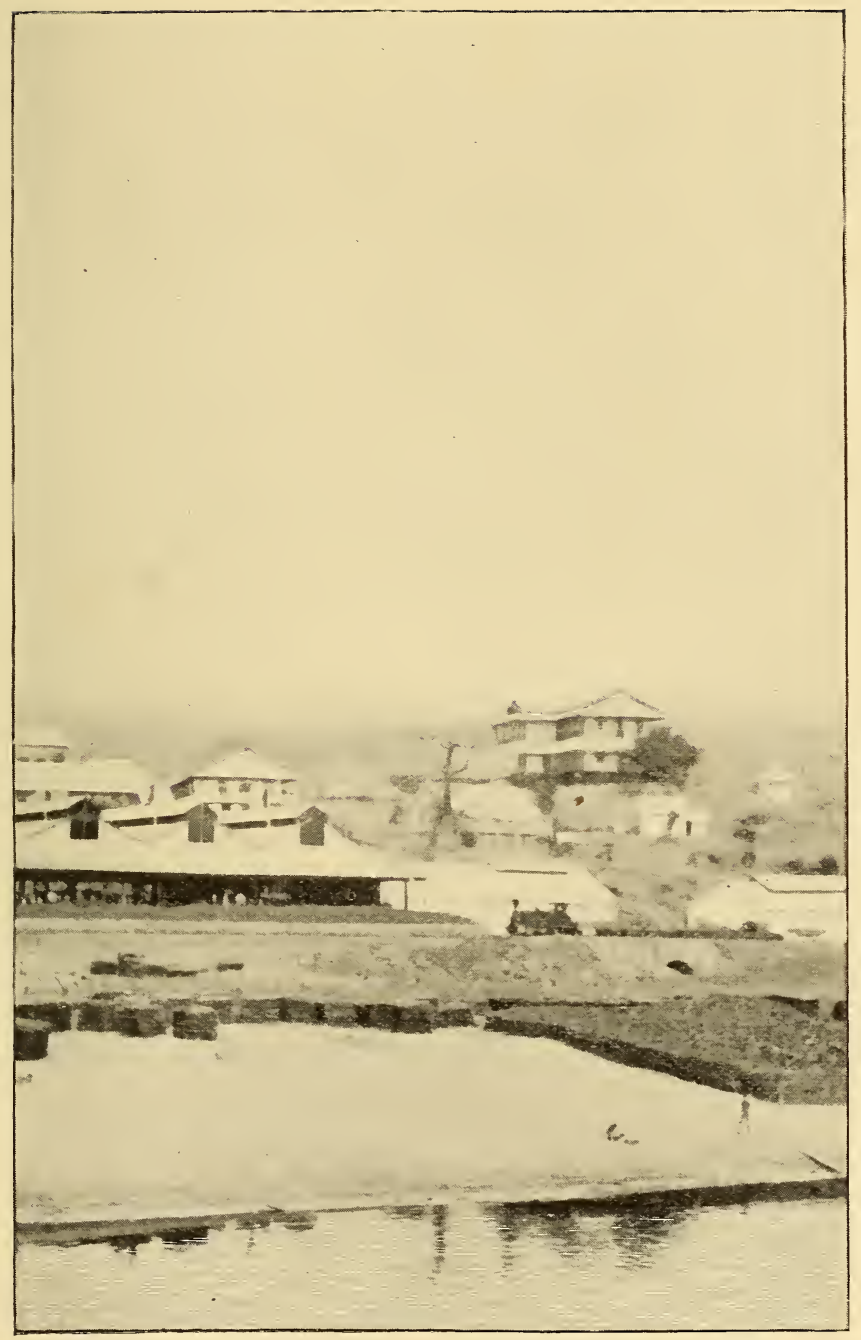

Matadi. 



\section{III}

\section{LE CHEMIN DE FER}

Deux journées de voyage, de Matadi à Léopoldville, gravent dans la mémoire des images précises qui, infiniment mieux que n'importe quels arguments abstraits, font ressortir la grandeur et l'utilité des efforts accomplis pour substituer la nouvelle voie de communication à l'ancienne route des caravanes. Bien que le chemin de fer ait cause gagnée derant l'opinion, il est permis de rappeler que certains l'ont systématiquement décrié ou méconnu pendant toute la période de construction. A ces professionnels du dénigrement il est bon de dire et de répéter: "Je l'ai vu, de mes yeux vu. " Nombreux sont ceux, du reste, qui aujourd'hui peuvent rapporter 
pareil témoignage, écrit ou verbal. Peut-être n'y a-t-il guère à ajouter aux relations qui, traitant de la matière, l'ont épuisée. Mais dans une esquisse, aussi sommaire et incomplète soit-elle, du Congo belge, comment ne rendrait-on pas hommage, en passant, au travail prodigieux qui s'est effectué dans la région des cataractes, comment n'exprimerait-on pas l'admiration profonde dont on est pénétré à sa vue? Ayant obtenu, en outre, l'enviable faveur de pousser une pointe sur le HautCongo, nous ne saurions oublier que sans la voie ferrée amorcée sur le "Bas " cette excursion n'eût pas été possible. En touriste reconnaissant nous fixerons donc ici les impressions qui nous ont été communes avec nos compagnons de route de l'Albertville, encore qu'elles soient vouées à une publicité tardive.

Le lundi, 4 juillet, au point du jour, les trains de " l'inauguration " étaient rangés en gare de Matadi, sous pression, prêts à partir. Au nombre de six ils représentaient excellemment le " matériel roulant " de la ligne. Flambant neuf, machines et voitures répondent à la description qui nous en a été précédemment donnée, celles-là de formes massives et trapues, celles-ci de dimensions que ne laisse guère soupçonner l'écartement minime de la voie : un gros chemin de fer en vérité et non le tramuay joujou de la légende.

Chaque locomotive est attelée de trois wagons dont l'un à ciel ouvert sert de fourgon à bagages ou... à nègres, indifféremment, les deux autres étant réservés aux voyageurs de qualité, aux blancs. Très coquet, l'intérieur de ces premières classes, avec leurs sièges 
d'osier, sur pivot, disposés latéralement, avec leurs petites tables mobiles, fixées aux parois, ces dernières arrêtées à hauteur dappui entre les montants qui soutiennent le toit : fenêtres toujours ouvertes. Des stores épais protégeront, le cas échéant, contre les intempéries du dehors, la fumée, la poussière et surtout contre l'ardeur du soleil. Allègrement, "en beauté " les trains s'ébranlent pour se suivre à la file, à vingt minutes d'intervalle.

Dès après la sortie de Matadi surgissent les formidables défenses que les ingénieurs durent briser ou tourner. Côtoyant d'abord le Congo et, par instants, le surplombant à pic d'une étroite corniche, la voie court accrochée au roc dans lequel elle est taillée.

Plus loin, ayant franchi la Pozo, elle s'insinue en courbes et contre-courbes, suivant les méandres de la rivière. Encore quelques kilomètres et c'est l'attaque du redoutable massif du Palabala sur les contreforts duquel il lui faut s'élever graduellement, à travers un dédale de fonds et de crêtes, houle géante figée dans une morne et sombre immobilité.

On conçoit alors les tâtonnements du début, l’hésitation dans le choix d'une direction, sans compter les difficultés inouïes d'exécution lors de l'établissement définitif de la plate-forme.

Du point de vue pittoresque, - celui du voyageur contemplant à loisir, - tout cela est d'ailleurs admirable. L'attention est sans cesse sollicitée par d'étonnants changements de décors : entassement de rochers au-dessus de soi, torrent bruissant au-dessous, pentes 
chauves où s'allongent des serpents de verdure, bosquets touffus dans les creux qui ont recueilli l'eau vivifiante. De-ci de-là des appellations évocatrices : Le " pic Cambier ", le " ravin du Diable ", le " ravin du Sommeil ", jusqu'à ce nom d'espérance et de victoire, le " col de l'Horizon ", d'où le regard peut embrasser l'étendue.

L'on n'est parvenu qu'au kilomètre 25 , et c'est cependant une étape énorme qu'on laisse derrière soi.

Intérêt et curiosité s'avivent encore des souvenirs personnels d'un des plus notables ouvriers de la première heure, le major Cambier qui, présent parmi nous, rappelle à maint tournant de la route quelque fait de la mission d'études dont il fut le chef. C'est ainsi qu'arrivé à l'endroit dénommé " col aux Buffles ", il raconte l'aventure d'un de ses collaborateurs. Travaillant à un lever de terrain, celui-ci fut chargé par un solitaire. Fuite du porte-mire et des autres noirs. Que faire, sans armes? Restant immobile, le blanc eut la présence d'esprit de souffler à pleins poumons dans son cornet d'appel. Ce bruit insolite surprit l'animal qui tourna court. A dix ans de distance, de deux frères l'un fut le héros de l'histoire, l'autre en écouta simplement le récit : rapprochement inattendu et, sur les lieux mêmes, un frisson d'émotion.

Tandis qu'on regarde, qu'on devise, en cherchant à arrêter, dans les sites mouvants, les détails essentiels. l'on avance toujours. L'allure de notre train est d'une régularité parfaite, sans heurt ni secousse, et il en est exactement de même des trains qui nous précèdent et de ceux qui nous suivent. La vitesse à l'heure atteint 
environ 20 à 25 kilomètres, moyenne très satisfaisante en terrain aussi accidenté.

Que reste-t-il alors de tant de critiques fantaisistes? $\mathrm{Au}$ lieu des déraillements pronostiqués, une stabilité entière; au lieu des éboulis menaçant de droite ou de gauche, des talus et des tranchées solidement établis, un profil général inspirant toute sécurité. La définition nous revient à l'esprit de la "musculature " puissante de ce "sentier de fer " avec les détails techniques qui, sur place, acquièrent leur pleine valeur : les rails pesant 2 I kilogs au mètre, les traverses métalliques de 36 à 37 kilogs, à distance de $\mathrm{o}^{\mathrm{m}}, 75$ environ d'axe en axe, au total roo kilogs de fer par mètre courant, membrure bien faite pour supporter et assurer l'adhérence de machines de 30 tonnes et de wagons de io tonnes. A des conditions particulières ont répondu les moyens correspondants. L'improvisation et le hasard ne comptent pour rien dans la réussite finale. Si des fautes, d'ailleurs réparables, ont été commises, conception et exécution se sont inspirées de vues manifestement judicieuses.

Le côté " exploitation " ne paraît pas moins bien ordonné. Aux gares et haltes, qui sont nombreuses pour permettre aux machines de s'alimenter d'eau, le personnel se tient exact au poste, la manœuvre s'exécute rapidement.

Attrayants souvent ces jalons de civilisation industrielle. Autour de l'habitation du chef, coquettement parée, des jardins naissants. Au lieu des légumes et des fleurs, choux et roses rassemblés en quelque modeste plate-bande, l'on aperçoit des ananas, des raphias, sur- 
tout des bananiers, mais dans ce cadre, si différent de ceux qui nous sont familiers, même souci de soin et de minutie. Des blancs sont installés là qui ont importé leurs habitudes; mais la tenue n'est pas moins louable chez certains de leurs collègues de couleur ; à témoin ce noir qui, lorgnon à l'œil, carnet en mains, vint saluer les autorités, prendre et donner des ordres comme l'eût fait un chef de station de grande banlieue.

A ce propos que penser de la main-d'œuvre indigène, ou, en termes plus généraux, de l'utilisation du noir dans les entreprises de la civilisation? La question se pose, au seuil de l'Afrique, singulièrement pressante. Y chercher réponse est presque un devoir pour tout voyageur désireux d'information.

$\mathrm{Au}$ chemin de fer elle a été résolue, à n'en pas douter, de façon décisive. C'est le premier cas concret qui se présente. En attendant qu'il soit corroboré par d'autres infiniment nombreux, interrogeons Ce nous est chose aisée, car M. De Backer, un ancien, et le directeur désigné de l'exploitation, se trouve là qui très obligeamment nous renseigne.

Pour la construction de la ligne on recourut aux " gens de la côte ", ainsi qu'on nomme en bloc les noirs de Guinée, qu'ils ressortissent aux possessions françaises, anglaises ou portugaises. Sierra-Leone, Accra, Lagos fournirent les plus gros contingents. Au-dessus d'eux, faisant bande à part, orgueilleux, chauvins et turbulents, mais vigoureux, sobres, énergiques, les Sénégalais. Ils sont difficiles à mener, mais une fois que vous les " tenez en mains ", que vous leur faites accepter votre 
autorité, ils se montrent travailleurs émérites. On leur doit notamment les terrassements d'avancement si rapide dans la dernière période de l'entreprise. Parmi eux aussi se recrutent des employés et la plupart des machinistes de locomotives. L'honneur leur échut de conduire les trains de l'inauguration. Si chacun d'eux est doublé d'un mécanicien blanc, ce dernier surveille l'engin, mais ne le dirige pas.

Quant aux indigènes proprement dits, les tâches faciles leur ont été confiées, celles d'aides-tâcherons, ainsi apporter des matériaux à pied d'œuvre. Mais leur rôle a crû insensiblement. Pour l'entretien de la ligne ils sont dès à présent suffisamment préparés. A cet effet on s'est adressé aux mieux doués des enfants, à l'âge où ils sont malléables. Ce sera laffaire d'une génération ou deux pour qu'ils puissent rendre les mêmes services que leurs aînés de la côte. Près de nous, le serre-frein, assis dans une petite guérite en porte-à-faux, est précisément un Bacongo qui, avec vigilance, exécute sa besogne de serrage et de démarrage sur les pentes et les rampes. Nulle crainte qu'il ne s'oublie ou ne s'endorme.

A chacun selon ses mérites. Les salaires varient beaucoup suivant les catégories de travailleurs. Les noirs du pays reçoivent fr. $0-25$, fr. $0-50$, fr. $0-75$ par jour, tandis que les gens de la côte gagnent i franc à fr. I-5o et les ouvriers supérieurs, tels les machinistes, jusqu'à Io francs par jour.

Ainsi se réalise, soit par l'initiation directe, soit par l'exemple d'autres nègres plus avancés, l'éducation des Congolais. Tâche particulièrement ardue, semée 
d'écueils, soumise à de périlleuses incertitudes. La main-d'œuvre faillit un instant faire défaut au chemin de fer; le sort de l'entreprise en dépendait. Des noirs seuls, après d'infructueux essais de recrutement chez les Asiatiques ou aux Barbades, la fournirent en fin de compte, et dans des conditions hautement satisfaisantes. $\mathrm{Si}$, parmi ces noirs, il convient d'admettre une certaine classification, une forme d'hiérarchie, il n'est plus exact d'affirmer qu'une distance infranchissable les sépare. Au contraire, la preuve est faite qu'au Congo même les bras sont éducables, si j'ose ainsi parler, pour ne point préjuger encore la question infiniment plus délicate de la perfectibilité morale.

L'homme, même en ce siècle de machinisme et de progrès industriel, reste toujours à la base de tout effort. Convertir en instrument utile l'être végétatif qu'il est à l'état sauvage, est peut-être le problème le plus ardu à résoudre. C'est pourquoi, à l'occasion d'une entreprise qui a exigé tant de science du blanc civilisé, il est permis encore d'attribuer la première place à sa mission propre d'entraîneur et de conducteur d'hommes. Les œuvres sorties de son génie inventif ne viennent qu'après comme la conséquence de sa primauté intellectuelle. Il y a d'ailleurs un charme surprenant à les rencontrer dans le cadre d'une nature jusque-là indomptée. Le terme de travaux d'art prend du coup une signification à la fois plus haute et plus vraie. Sans doute les constructions de nos chemins d'Europe, ponts, tunnels, viaducs, gares, présentent des proportions en soi autrement imposantes. Les portées de 60,80 , 100 mètres 


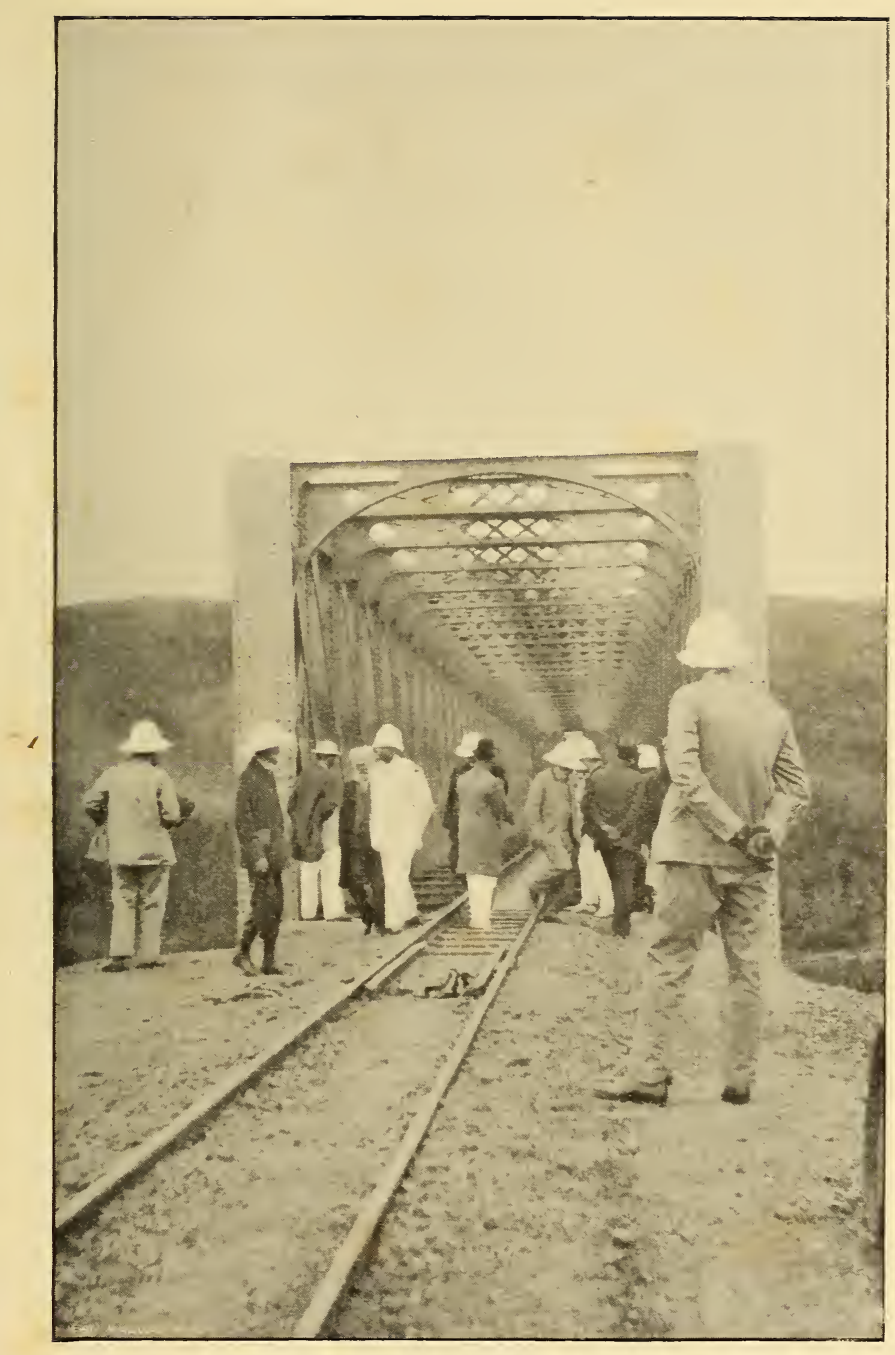

Le Pont de l'Inkisi. 

des plus importantes rivières de la ligne congolaise, en leurs points de passage, n'ont rien d'excessif. Mais pour mesurer les ponts qui les franchissent, il y a, en outre, l'échelle de la difficulté vaincue, d'un ordre tout particulier en Afrique.

Après la Pozo et le Kwilu, on rencontre l'Inkisi, qui est le plus large de ces cours d'eaux. A raison de ses fortes crues, durantlesquelles il monte de 7 mètres, développant une vitesse de courant terrible, charriant comme des fétus pierres et arbres, le tablier métallique, complètement monté, dut être lancé d'une pièce d'une rive à l'autre. Disparus aujourd'hui les supports provisoires, têtes de pilots, sur lesquels on le fit glisser. La solution seule apparaît en son élégante simplicité : le treillis de fer ajouré, léger et robuste dominant de haut la rivière qui, sans prise sur lui, coule impuissante en ses éventuelles colères. Travaux d'art encore et surtout, les multiples et ingénieuses combinaisons imaginées pour épargner ceux que techniquement on nomme ainsi. Montant, descendant, semblant revenir sur ses pas lorsqu'elle s'élève à un palier supérieur, la voie épouse agilement toutes les sinuosités du terrain.

Si l'ingénieur a trouvé son compte à ce mode de cheminement, le voyageur, pour sa part, n'a pas à le regretter. Le paysage se déroule littéralement sous ses yeux, sans les interruptions inattendues et souvent brutales des tunnels ou des profondes tranchées. Lorsque se dresse une montagne, la ligne la contourne, en opère l'ascension, sauf à gagner prestement la vallée située au bas du versant opposé. 
Parmi tant de paysages, il en est deux qui exercent comme un prestige supérieur.

Au delà de la station de Kimpese blottie dans un de ces nombreux replis de terrain que jalonnent les circuits si hardis de la voie de fer, s'élève la chaîne du Bangu, dont la silhouette massive domine tout un temps une campagne à ondulations lentes. Se profilant dans deux directions qui se coupent à angle droit, elle présente des silhouettes qui vaguement rappellent quelque préhistorique pachyderme, au dos énorme et rugueux, tant les assises en sont pesantes, et le faîte dessiné en ligne continue. L'entrecroisement des crêtes d'arrière-plan, la structure tassée du mont ajoutent à sa grandeur qui se maintient jusque dans l'éloignement.

Non moins majestueux, le site de Zona-Congo, point culminant de la ligne, à 700 mètres d'altitude. D'un effort patient, les lacets superposés à la montée élargissent au fur et à mesure le champ de vue. Du col et durant la descente qui suit, les regards embrassent circulairement des lointains aux limites devenues indécises. En la matinée du 5 juillet la vaste étendue était baignée d'une lumière fine et rare. La rosée épandue par l'aurore gardait encore la brousse des morsures trop cruelles du soleil. La chaleur ne brûlait ni ne désolait; elle enveloppait seulement la terre de vibrations transparentes.

Par monts et par vaux, telle reste l'allure générale du tracé, bien au delà du Palabala, qui dut sa fâcheuse réputation au fait qu'il barra trop tôt la route aux travailleurs de la ligne. Avec l'expérience venue, les bonds en avant, dans l'assaut donné à la nature rebelle, furent 
assurément mieux réglés et plus lestement exécutés.

Quand ce n'est pas le sol en sa structure foncière qui oppose de la résistance, il faut encore compter avec celle des végétaux hostiles. Ce serait en effet une erreur de croire que la traversée des monts de Cristal ne comporte que pierres et aridité. A maintes reprises déjà nous avons eu l'occasion de signaler ces îlots de verdure luxuriante où certains de nos plus beaux bois entreraient tout entiers. Ainsi surtout, de la forêt de la Lukaya, la rivière qui donne la direction au rail au delà de l'Inkisi, jusqu'aux approches du Pool. La hache dut cogner dur pour frayer ici un passage. Aujourd'hui, à travers ces antres de noire ramure, capables en certains endroits, croirait-on, de se refermer sur celui qui y pénètre, la locomotive s'élance librement, gagne l'éclaircie prochaine pour refaire plus loin de nouvelles trouées. Prêtez-leur vie et mouvement - plus qu'ils n'ont l'air d'en avoir en menant leur ronde aux côtés du train qui passe - à tous ces êtres inanimés : arbres aux troncs noueux, plantes aux dards aigus, lianes qui, tels les mythiques serpents, semblent prêtes à étrangler, et parmi ces formes hirsutes, sous ces attitudes de violence, quelle furieuse mêlée! Cependant, au roulement des pesants véhicules, et quand siffle la machine, quelque singe apeuré saute de branche en branche jusqu'à ce que renaisse le calme harmonieux qu'anime seul le souffle léger du vent dans les feuilles. La volonté consciente et agissante a commandé la soumission.

Quand, après tous ces détours, la voie ferrée accède à la plaine terminale qui borde le Stanley-Pool et que, 
rigide, elle suit, pendant plusieurs lieues, la ligne droite, c'est un autre charme qui opère sur l'esprit. Inconsciemment l'on revit la joie des hommes, maîtres de l'entreprise, qui la firent aboutir. Quelle douceur alors, dans cet horizon aplani, - aux tons maussades pourtant, - qui symbolise la fin des peines et des rudes travaux. Quelle délicieuse sensation de voir poindre, auprès des gigantesques baobabs de la rive, l'eau grise et calme, miroir fidèle d'espérances réalisées. Le port ne fut jamais plus ardemment désiré du marin. Et n'est-il d'ailleurs pas un havre, ce lac où se réunissent, semblables à ceux d'une mer, les flots du Congo, où se prolonge, par le canal de la nouvelle voie, l'océan.

Il n'est pas d'indifférence qui eût tenu contre les impressions de cette inauguration triomphale. En ses différentes phases elle procéda comme une véritable marche à l'étoile. Un air de fête soufflait qui gaiement montait au cerveau. Sur tout le parcours ce n'étaient que drapeaux, présentations d'honneur, félicitations, hurrahs! Jusqu'aux enfants, bonshommes et fillettes de la mission de Kimuenza, qui, rangés par les pères et les sœurs au passage de notre train, le firent s'arrêter. Les autorités descendirent, complimentèrent et, en se rembarquant, furent saluées de joyeuses acclamations.

Autre épisode du voyage, aussi haut en couleurs qu'amusant : l'arrivée et l'installation à Tumba, au soir de la première étape. On eût pensé assister à une représentation machinée par le plus habile des metteurs en scène. Sur le plateau, très étendu, où est située la gare, les chefs du voisinage s'étaient donné rendez-vous pour 
accueillir dignement les blancs illustres. Le cortège officiel passa entre deux haies merveilleusement composées. Peaux de léopards et de crocodiles portées haut en guise d'enseignes, pagnes multicolores, couvertures de laine, défroques invraisemblables où l'uniforme rouge de quelque officier anglais voisinait arec le casque flamboyant du plus légendaire des pompiers, parasols à teintes bariolées, tout cela formant le plus hétéroclite assemblage qu'on pût rêver. Et la multitude noire de se démener, de crier, de constituer une, foule n'ayant que de vagues analogies avec le chœur antique, mais conservant parmi ces accessoires burlesques des airs de dignité sauvage et primitive.

Une autre surprise attendait les invités : le village en bois érigé à leur intention, sorti de terre, comme par un coup de baguette magique, hospitalier à souhait. Au centre de ces habitations improvisées, un hall de belle dimension orné et paré pour le banquet du soir. Le colonel Thys y présida, donnant, ainsi qu'à l'habitude, l'exemple des allocutions chaleureusement applaudies. La nuit venue, par un clair de lune d'argent bleuté, chacun put, en son logis, continuer le rêve de la journée, s'il ne préférait se rendre à l'invitation des tamtams qui battaient une frénétique chamade, et assister aux danses les plus authentiquement indigènes, comme inspirées de la Vénus, callipyge ou ventripède à volonté, la moins effarouchée.

Les fêtes de linauguration trouvèrent leur conclusion dans la journée du 6 juillet. Matinée et après-midi avaient été employées à visiter les installations déjà con- 
sidérables de Léopoldville, son voisinage immédiat, sur le lac, et sur la rive française, en face, Brazzaville, dont $M$. de Lamothe fit les honneurs avec une bonne grâce parfaite en nous ménageant la surprise d'un cortège bateke, de pittoresque rutilant.

Devenus les hôtes de l'État, passagers de l'Albertville et résidents du Congo étaient réunis le soir sur la veranda du mess, maison commune au centre de la cité. C'est là, dans un cadre des plus frustes, que furent prononcées les harangues officielles.

Sanction gouvernementale imprimée à l'entreprise du chemin de fer, témoignages de sympathique solidarité des puissances étrangères, tel en était le sens attendu et obligé. Cependant elles marquèrent comme le point culrninant de ces belles journées. Lorsqu'au nom de l'État Indépendant M. Fuchs, faisant fonctions de gouverneur général, déclara la ligne de Matadi au Stanley-Pool ouverte à la libre circulation, il se plut très justement à évoquer le passé, récent mais si fécond en hauts faits, de toute l'œuvre congolaise. A larges traits il en reprit les origines, entièrement issues d'une pensée de génie, en rappela les phases décisives, pour déterminer ensuite la place importante qui revient au percement de la région des cataractes dans ces vingt années de virils efforts.

Honneur aux promoteurs de l'entreprise, à leur plus éminent représentant, le colonel Thys, honneur à ses dévoués collaborateurs, le major Cambier, MM. Espanet et Goffin, pour ne citer que les principaux. Honneur enfin aux disparus, trop nombreux, hélas! qui payèrent de la vie leur zèle et leur dévouement. 
Et d'entendre ces paroles à la fois fières et de gravité émue, on sentait qu'elles devaient retentir bien au delà du cercle étroit des auditeurs. En Belgique d'abord, le pays premier intéressé aux succès de ses enfants, et dans le monde entier, où, du fait du Congo, elle prend une place que trop rarement chez nous on veut considérer. Le comte d'Ursel, délégué du gouvernement belge, en un discours plein de sens patriotique, M. von Danckelmann, au nom du gouvernement impérial allemand, furent les interprètes autorisés de cette pensée du dehors en laquelle les "Congolais " trouvent sujet à si légitime satisfaction. Non moins vibrante et spontanée fut l'impression qui jaillit des toasts prononcés durant le banquet. Dans cette assemblée si diversement composée, où voisinaient délégués étrangers, officiers, fonctionnaires, missionnaires, journalistes, passa un frémissement d'unanime admiration quand, exaltant son œuvre, le colonel Thys la proclama grande entre toutes, puisqu'à chaque bout le chemin de fer tient un monde : la barbarie et la civilisation. Le geste et le verbe puissants de l'orateur lui conféraient un prestige certain, dont, par une sorte de choc en retour, nous tous, ses concitoyens, devenions en cette heure les bénéficiaires. Très naturellement la fête tourna à l'apothéose, au bout de cette excursion menée, avec un brio incomparable, d'Anvers jusqu'au feu dartifice terminal qui illumina de ses gerbes les eaux du lac de Stanley.

... Mais voici qu'a sonné l'heure de la séparation. La plupart reprennent le chemin de l'Europe. A trois, M. Buls, M. Nyst et moi, nous poursuivons plus avant. 
D'autres spectacles de vie indigène nous attendent, des paysages inédits vont défiler sous nos yeux. A notre tour, nous pourrons soulever un coin du voile qui couvre l'Afrique mystérieuse. Quel que soit l'attrait prestigieux de cet inconnu qui s'entr'ouvre, nous compterons parmi nos meilleurs souvenirs la traversée de l'Albertville et les journées désormais fameuses de l'inauguration du chemin de fer.

Nous en savons infiniment gré à ceux qui s'en firent les très courtois dispensateurs. 


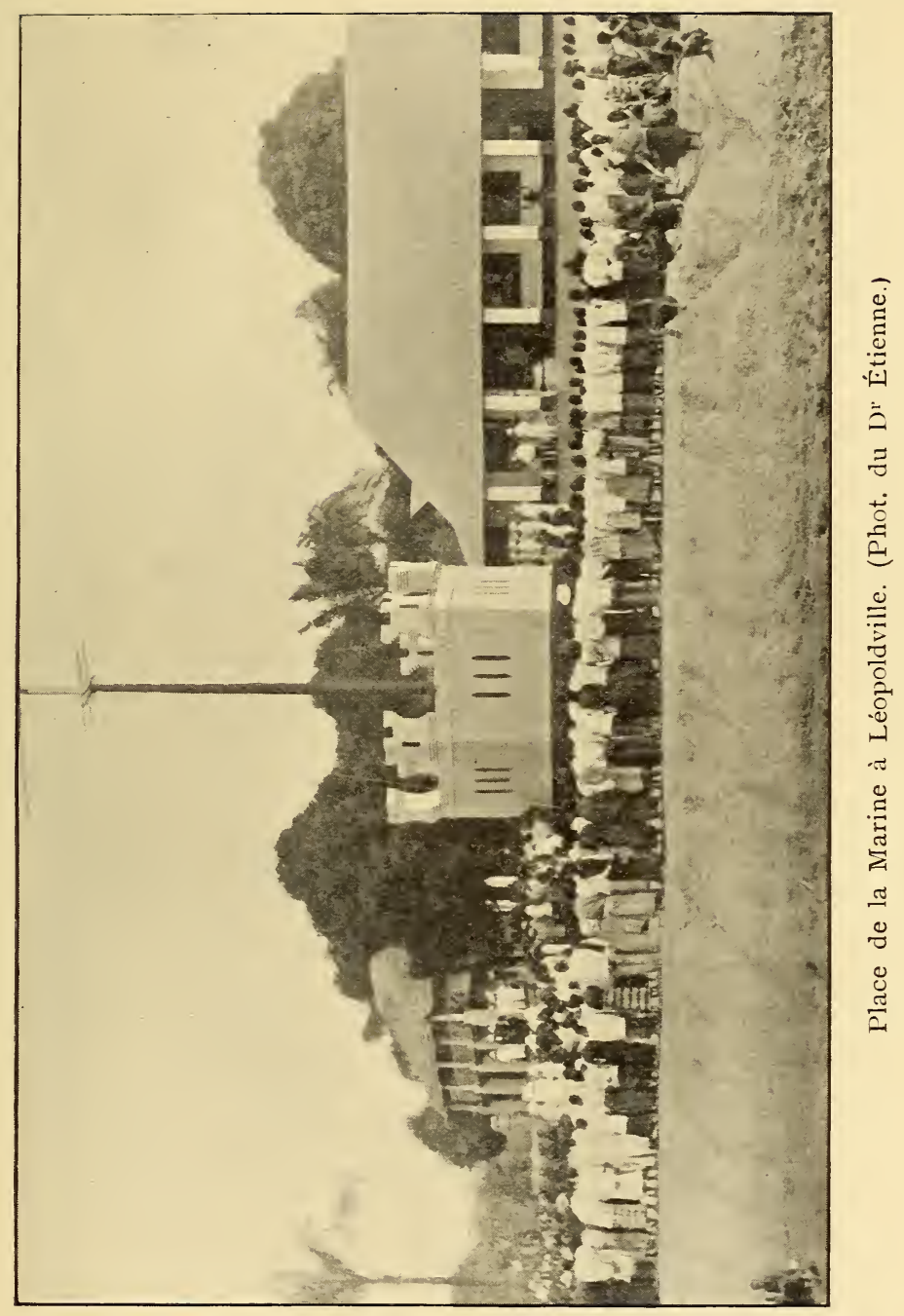





\section{LE STANLEY-POOL}

Lorsque le voyageur, montant du Bas-Congo par le chemin de fer, ou descendant du "Haut " par le fleuve même, débouche sur la belle nappe d'eau que Stanley qualifia modestement de $\mathrm{Pool}$, il a vue sur un vrai lac (450 kilomètres carrés) occupant, à un cinquième près, une superficie comparable à celle du lac de Constance. Le Stanley-Pool, à sa limite d'expansion la plus prononcée, ne laisse point d'une rive découvrir la rive opposée. Il ne faut pas moins d'un demi-jour de navigation à vapeur pour le parcourir dans le sens de sa longueur. Par l'île de Bamu, placée en son milieu, par d'autres îles de moindre étendue, il est 
divisé en sections considérables, quoique d'importance inégale. Il recueille presque toutes les eaux issues en si grande abondance du versant occidental du centre africain et il les retient jusqu'à ce qu'elles se frayent de force et par bonds successifs un passage à travers la région des cataractes. Les monts de Cristal l'enserrent d'une ceinture plus ou moins lâche, dont les contreforts les plus escarpés sont situés près du " chenal " à l'entrée. Le seuil d'aval, tranchée tourmentée que le fleuve s'est creusée à la sortie, est d'altitude moindre. Les Dover Cliffs, du côté de l'est, avec leurs pans de granit rayés de zébrures blanches qui les font ressembler effectivement aux falaises crayeuses de la côte anglaise, sont remplacées, à l'ouest, par les croupes arrondies des environs de Léopoldville.

Le rôle de ce réservoir admirable est trop clairement marqué par la nature, pour qu'il échappe aux yeux de l'observateur le moins attentif. En son entier il forme rade ou port. Deux fois tête de ligne, il commande, en le desservant, le réseau des voies navigables de l'intérieur, véritable Méditerranée, alors que d'autre part il est le point d'aboutissement obligé de la route maritime prolongée par le chemin de fer.

Entrepôt général et lieu de transit, le Stanley-Pool a pour objet principal d'amorcer toutes les communications avec l'intérieur. Pour l'État, personne publique, comme pour les compagnies, personnes privées, il est le siège initial de leurs transactions. Ici leurs intérêts ou bien se confondent ou bien accusent certaines divergences. Sur place s'opérera le règlement le plus prompt 
et donc le plus avantageux des questions qui peuvent se présenter.

En ce carrefour de grand'route fluviale viennent encore converger des éléments politiques. La France, voisine de l'État Indépendant, partage avec lui la propriété des eaux du lac. Qu'on est loin de l'époque où Stanley et de Brazza se rencontraient en rivaux sur ses bords. Aujourd'hui les rapports entretenus par les deux puissances sont des plus cordiaux. Il y a passage continu d'une rive à l'autre, surtout depuis que le Congo français se sert de la voie ferrée construite sur notre territoire. N'empêche que les relations de frontières demandent toujours une attention particulière. Le cas échéant, les incidents du "Haut " sont rapportés sous un jour différent suivant qu'on se trouve à Léopoldville ou à Brazzaville. C'est ici que se perçoivent les premiers échos des bruits fâcheux qui naissent, se propagent et s'enflent si aisément aux pays du soleil. Il incombe alors aux hommes de direction de conserver, avec le sentiment de leur dignité, tact et sang-froid.

Tout, dans la situation du Stanley-Pool, concourt, on le voit, à lui assigner une importance capitale. Pour peu que l'État Indépendant se développât, des agglomérations devaient se constituer sur ses bords, amenant avec elles les besoins de toute communauté civilisée, et l'institution des rouages propres à les régler. Adminis. tration, force publique, travaux d'utilité, cultures et, dominant l'ensemble en pays neuf, l'organisation des services de transport et de ravitaillement, tels sont les régulateurs obligés de la vie en société. 
Eh bien! c'est tout cela qu'on trouve réuni dès à présent à Léopoldville, à la période, cela s'entend, de la croissance.

On ne peut qu'être surpris de la méthode et de l'activité qui ont présidé à la création de la cité. De belles avenues ont été tracées de la rive jusqu'à mi-hauteur de la colline à laquelle elle est adossée. Dans leur répartition il y a comme deux étages. Au palier inférieur, dans l'" allée du Roi-Souverain ", sur la " place Stanley ") sont érigées des bâtisses en briques. Des préoccupations monumentales se sont déjà fait jour. Ainsi de l'édifice qui sert à la fois de mess aux agents blancs et de palais de justice. Ainsi encore de la colonne, œuvre du capitaine von Irgensberg. Au socle, le médaillon de Léopold II ; au sommet, une négresse largement épanouie qui, le flambeau à la main, symbolise l'appel à la liberté, à la lumière et à la civilisation.

Sur le palier supérieur, la " ville haute ", des manguiers alignés en longues files cachent dans leur ombrage des maisons de style plus primitif, séparées les unes des autres. L' " avenue de la Terrasse ", d'où la vue porte sur le lac, l'" avenue du Camp militaire » ont leur charme et leur coquetterie. Reliant entre eux les deux quartiers, des raidillons nombreux. Terminés en marches d'escalier, ils sont soutenus par des fascines et protégés en outre contre le glissement des terres par de profondes rigoles latérales.

Pour fouiller le sol, tracer les routes, planter, construire, on pense s'il a fallu peiner. Des résultats qui en Europe ne comptent pas, représentent là-bas une 


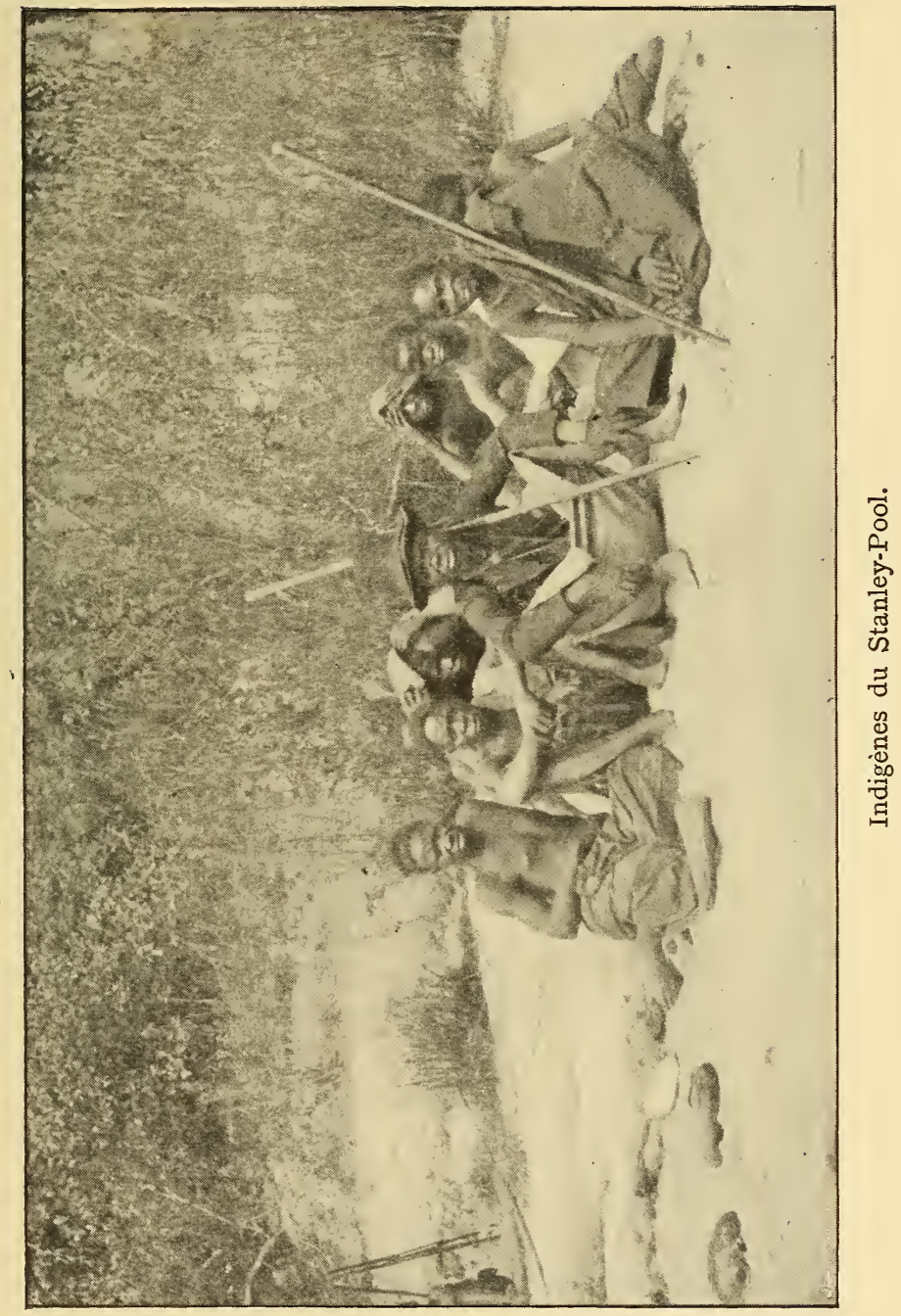



somme d'efforts énorme. Faire des briques, scier de long, ajuster, en un mot, travailler de façon quelconque, voilà ce qu'on a dû d'abord enseigner aux noirs. Dirigés par des blancs ou des gens de la côte, ils ont appris, ils savent parfois fort bien. Dans la force publique se recrutent généralement les artisans les plus habiles et les plus aptes, en raison de leurs habitudes de discipline, à fournir une besogne régulière. C'est tout un petit monde de travailleurs qu'une station comme Léopoldville doit mettre en mouvement, qu'elle les emploie à l'entretien des chemins, aux cultures, aux constructions. A leur tour ils réclament, pour leur alimentation, du travail de leurs congénères. C'est au marché institué par les blancs que les villages des environs livrent les vivres frais ou séchés, les fruits et légumes, surtout la chikwangue ou pâte de manioc, entrant dans la consommation courante des indigènes.

Quels sont les blancs, demandera-t-on, appelés à entretenir cette activité? On peut les répartir en deux catégories. La première comprend le personnel même du chef-lieu qui doit faire face à tant d'exigences et de nécèssités : le commissaire général de district et son adjoint, le juge, le médecin, l'intendant, les officiers de la force publique, le commandant du port et les capitaines de bateaux, l'ingénieur-mécanicien, l'employé des postes, puis les commis, contremaîtres, sous-officiers et ouvriers d'élite. La seconde catégorie se compose des " passagers ", c'est-à-dire des fonctionnaires, factoriens, voyageurs, qui attendent à Léopoldville leur tour de départ pour le Haut-Congo, ou qui sont à la veille de 
descendre à Matadi pour se faire rapatrier en Europe. Contingent variable mais qui grossit parfois dans une très forte proportion le nombre des blancs. La colonie entière se retrouve au mess, divisé en deux classes, auquel ses membres sont tenus, à de rares exceptions près, de prendre part. Les repas en commun ont du bon; en l'état actuel, il serait d'ailleurs difficile de recourir au système du ménage individuel, subordonné à l'installation préalable d'un commerce de détail plus ou moins complet. Tous réunis, les Européens atteignent, le cas échéant, au chiffre de cent cinquante.

Beaucoup d'entre eux, comme nous venons de le voir, sont exclusivement sollicités par le haut fleuve. Suivons-les à la rive du lac où se déploie une vie de travail qui est la caractéristique de Léopoldville. Nous sommes "place de la Marine " au centre de laquelle, émergeant d'une petite tour crénclée, s'élève le mât de pavillon qui porte haut le drapeau bleu étoilé d'or. En arrière de la berge, des constructions rectangulaires : ce sont les forges et ateliers de réparations qui sans cesse montent de nouveaux bateaux ou entretiennent ceux qui sont à flot. Un peu plus loin, s'abaissant suivant un plan incliné jusque dans l'eau, le slip, glissière formée de puissants madriers placés dans le sens de leur longueur en lignes parallèles. C'est là que sont assemblés et lancés les bateaux de la flottille imposante déjà de l'État. Les grosses pièces usinées en Europe arrivant maintenant sans encombre à Léopoldville, il a été possible d'augmenter de beaucoup le tonnage des bâtiments. De 35 ou 40 tonnes on a passé à 150 tonnes, 
jauge du Brabant, dont on a appris lentière réussite.

C'est un spectacle imprévu à coup sûr et qui à lui seul témoigne des efforts accomplis, que celui de ce mouvement de va-et-vient des vapeurs démarrant de la rive ou y abordant. Les uns emmènent les nouveaux arrivés, pleins de confiance. Ils transportent les articles de nos manufactures, des étoffes principalement, au goût des noirs, et aussi, pour les Européens, des vivres, des ravitaillements de toute espèce qu'il est impossible de se procurer là-bas. Les autres, les bateaux qui reviennent au port, ont aussi des passagers, les " fin-determe ", plus pâles et plus fatigués ceux-là, tout heureux de rentrer. La cargaison est d'aspect bien exotique: balles de caoutchouc et pointes d'ivoire notamment.

On pense si la manutention de toutes ces marchandises est hérissée de difficultés. Quoi qu'on ait fait, les moyens de transports sont encore insuffisants. Les réquisitions des stations de l'État, aussi bien que des factoreries, dépassent constamment la jauge disponible. Il faut arranger, diviser, combiner, prendre en dépôt, remettre à une expédition ultérieure. Et de cet encombrement jaillit en tous cas cette constatation consolante que mieux vaut pléthore que pénurie dans la demande. "Les affaires marchent."

A ce tableau il y a une ombre. Ce qui fut réalisé à Léopoldville, surtout dans les cinq, six dernières années, sous la direction de $M$. le commissaire général Costermans, mérite des éloges auxquels il serait difficile d'apporter des réserves. Mais rien ne prévaut contre certains obstacles de nature. Le sol est, sans conteste, très 
défectueux, composé comme il est d'une vraie poussière noire où, pendant six mois de l'année, on enfonce jusqu'aux chevilles. Chose plus grave, la crique qui sert de port est trop petite et d'accès dangereux, en ce qu'elle est beaucoup trop rapprochée des cataractes. Naguère, la Ville de Verviers s'est abîmée, par suite d'une fausse manœuvre, dans les chutes, et a péri corps et biens. C'est le seul sinistre de l'espèce qui se soit produit, mais à raison de l'augmentation de la flottille les risques croissent à proportion.

Il est certain, en toute hypothèse, que les raisons qui aux yeux de Stanley militaient en faveur de l'emplacement de Léopoldville n'existent plus. D'après les dires d'un témoin des travaux de l'illustre explorateur, celui-ci fut séduit par la valeur stratégique de la position, au regard de l'attitude équivoque, plutôt hostile, des indigènes et par la proximité de la route des caravanes partant de la première chute. En outre, la population était plus clairsemée, les vivres et les cultures se faisaient plus rares, à mesure qu'on remontait la rive du Pool.

Aujourd'hui, changement complet. La région est pacifiée au point qu'il n'est plus permis de croire à une attaque des indigènes sur un centre de l'importance et de la nature de Léopoldville. Comme ligne de communication et comme base de ravitaillement la ligne du chemin de fer s'est substituée à la route des caravanes. Enfin, quant aux ressources locales, il importe assez peu qu'on soit à quelques lieues de plus ou de moins de villages trop pauvres en tout état de cause pour subvenir aux besoins grandissants d'une agglomération relative- 
ment dense. Tôt ou tard les blancs devront prendre euxmêmes en mains les cultures vivrières et surtout l'élevage du petit ou du gros bétail dont ils ont besoin pour subsister, eux e tleur entourage noir. Aux centres déjà créés dans le Bas-Congo, et qui n'ont que l'inconvénient d'être hors de portée ou de nécessiter des transports trop coûteux, répondront d'autres centres à organiser dans le haut. A ce point de vue il est fort possible que le StanleyPool soit plus tard ravitaillé d'amont par la voie fluviale.

On en est encore loin, c'est bien évident. Mais dans l'hypothèse envisagée du déplacement du port de Léopoldville, peut-être sera-t-il nécessaire de ne tenir compte que des exigences maritimes, et non du désir, en soi fort légitime, de ne pas trop s'écarter du chef-lieu actuel, en s'arrêtant à Dolo, par exemple. En réalité, et malgré des profondeurs énormes à certains endroits, la navigabilité du Pool laisse à désirer sur bien des points où se rencontrent des fonds variables, des roches ou des bancs de sables. De là l'idée d'aller d'emblée près l'entrée du chenal chercher la meilleure berge et la meilleure eau. Tout cela est à l'examen et à l'étude d'hommes du métier. Une solution ne tardera sans doute pas à intervenir, à l'effet de tirer le parti le plus avantageux de cette magnifique position du Stanley-Pool. La plaine et les collines qui le bordent sont, il est vrai, pendant une grande partie de l'année une steppe inféconde, à l'exception de quelques parcelles privilégiées appropriées à la culture ou à l'élève du bétail, telles les plantations de café du plateau de Galiema. La valeur du Pool est ailleurs, dans sa destination maritime, dans sa faculté 
de drainer commercialement tout l'intérieur. Ainsi Léopoldville, après Boma et Matadi et plus encore que la première, constitue un centre de vie et d'activité, où aboutissent quelques-unes des plus essentielles fonctions de l'organisme. Mais pour saisir la colonisation dans ces applications et dans ses résultats, il faut aller plus haut, jusqu'au point où le soleil et la pluie concourent à entretenir une zone de végétation forestière perpétuellement renouvelée. 


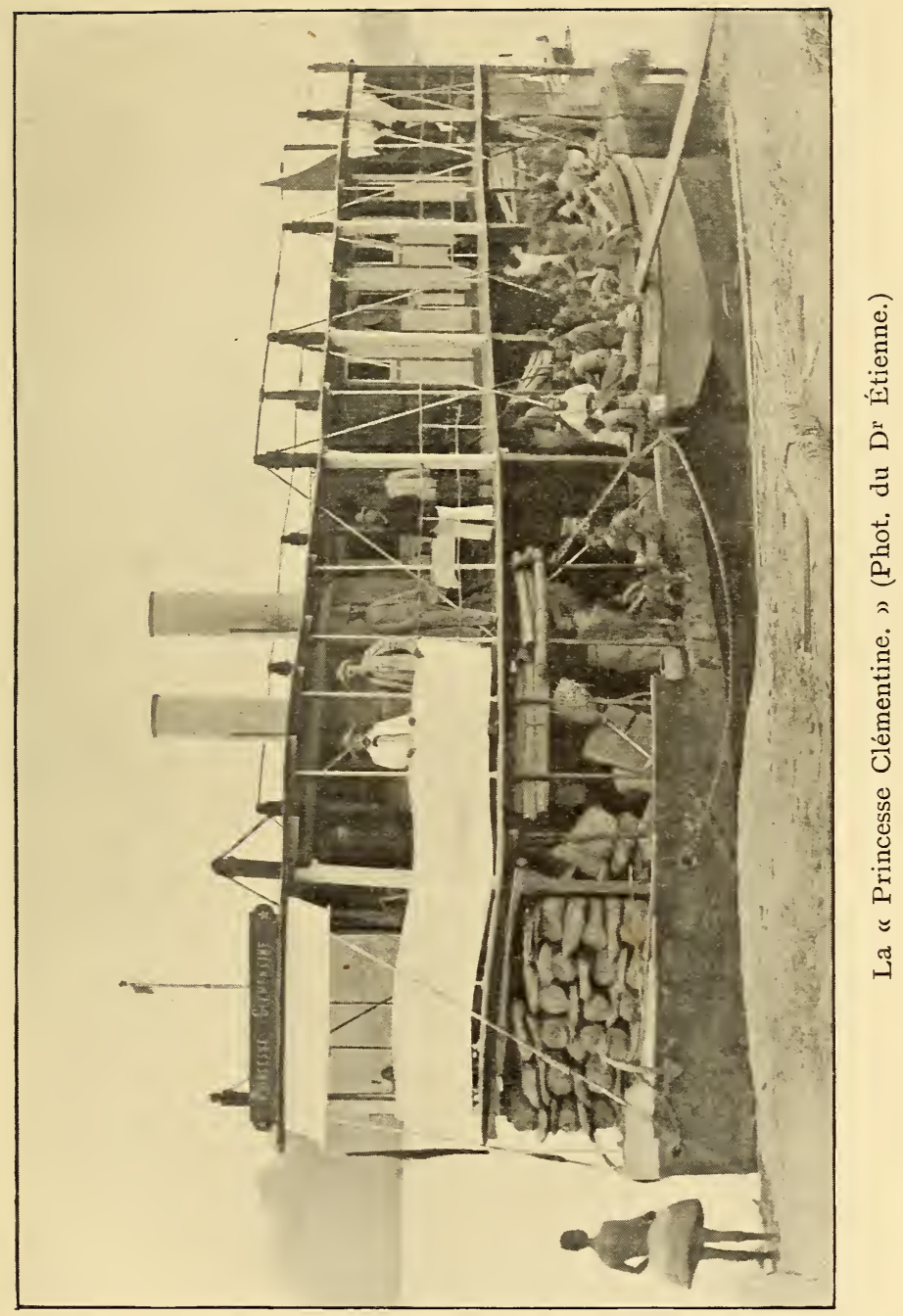





\section{V \\ LE HAUT FLEUVE}

Du Pool aux Falls la navigation libre d'obstacles comporte un parcours de r,60o kilomètres. En l'état actuel, il faut quarante jours pour le voyage, aller et retour. S'il n'était survenu un événement tout à fait fortuit, un choc malencontreux contre un rocher généralement recouvert d'une couche d'eau suffisante, c'est à bord du Brabant que les premiers touristes du haut Congo eussent accompli leur excursion. En somme, un faux départ, pour eux, et pour le bateau qui, dans la suite, racheta glorieusement sa faute.

Personne d'entre nous ne songea d'ailleurs à lui garder rancune pour la trop courte hospitalité quilil nous 
offrit du i i au r 3 juillet. Pendant ces quelques jours il nous révéla ses qualités qui sont réelles. Il se montra bon marcheur, docile aux impulsions du gouvernail, capable, tout en emportant une forte cargaison, quatre ou cinq fois supérieure à celle des vapeurs ses prédécesseurs, - d'assurer aux passagers confort et sécurité. Il nous brûla la politesse. Force nous fut de nous séparer de lui et de son très aimable commandant le capitaine Oppenrath.

Le bateau La Princesse Clémentine remplaça le Brabant. Et telle est l'inconstance humaine, nos affections se reportèrent aussitôt sur lui.

Un cadre intime était désormais assigné à nos pérégrinations fluviales. Qu'on s'imagine une demeure à deux compartiments superposés un peu à la manière de la terre et du ciel dans les anciens mystères : le rezde-chaussée, ou pont inférieur, en fer, qui dépasse à peine, surtout en pleine charge, le niveau de l'eau; l'étage ou pont supérieur, en bois, supporté par des piliers et des montants entre-croisés. Un toit léger le recouvre de bout en bout, en ne livrant passage qu'à dẹx cheminées jumelées. Au centre du bateau, un escalier en forme d'échelle établit l'unique communication, à moins qu'on ne préfère monter ou descendre, comme les gens de manœuvre, par les bastingages. Les habitants " d'en haut " sont peu nombreux. Outre le capitaine et son adjoint, quatre passagers, M. Buls, M. Nyst, le Dr Étienne et moi Un petit salon-salle à manger et la cabine du bourgmestre occupent le pont par le travers, tandis que leur font suite, accolées deux 
par deux sur la même largeur, les quatre cabines où s'installent les autres blancs, à l'exception du capitaine dont le logement à l'avant est adossé au coffre qui englobe la chaudière avec les accessoires.

En bas règne plus d'encombrement. L'équipage d'abord, composé de chauffeurs, coupeurs de bois, machinistes, quelques soldats-sentinelles; le personnel des serviteurs, cuisiniers et boys des blancs; des nègres à rapatrier, des femmes, des enfants. Dans le même espace encore, des chiens, des chèvres, des poules, des canards. Cependant la machinerie et la cabine du mécanicien blanc prennent une bonne partie de l'arrière, tandis que l'avant est tout entier occupé par la chaufferie, sans compter les deux fourneaux de cuisine.

Ajoutez à cela que chaque nègre possède son biloko, ensemble de mallettes, caisses, nattes, chaises, et concevez alors l'étonnant tohu-bohu qui règne dans cette grouillante smalah peuplée comme un village de quatrevingts, cent, cent cinquante habitants.

La cave, les cales dans l'espèce, sert à emmagasiner les marchandises et approvisionnements, ainsi que les quantités de bois nécessaires à l'alimentation des feux de la chaudière. Le cas échéant, ce bois se fait envahissant au plus haut point. S'il faut fournir une longue étape d'une seule haleine, des piles et des piles de bûches sont mises à bord. Elles flambent du reste comme allumettes, sous la flamme vorace, englouties par la bête à l'énorme ventre que les indigènes croyaient voir au début dans chaque machua remontant le fleuve.

Afin de dégager un peu le bateau, une assez grosse 
allège est amarrée solidement sur l'un de ses flancs. Elle reçoit une partie de la cargaison ou du bois; et les moins bien lotis d'entre les noirs ont vite fait d'y prendre place également. Il ne leur faut pas grand'chose, pour se sentir à l'aise, ainsi pour s'assoupir paisiblement sur une petite poutre en porte-à-faux, d'où le moindre mouvement peut faire choir le dormeur à l'eau.

Quoiqu'ils soient tous placés en bloc sous l'autorité du capitaine du bateau, ces noirs sont soumis à une certaine organisation hiérarchique qui les différencie les uns des autres, en attribuant une part du commandement aux mieux doués d'entre eux. Il y a le "capita " des bûcherons ou coupeurs de bois, le " capita " des gens d'équipage, le "capita " des chauffeurs; ce sont de véritables contremaîtres, qui indiquent la besogne à faire, qui la dirigent et la surveillent, mais qui ne mettent pas eux-mêmes la main à l'ouvrage. Leur action sur leurs hommes est parfois singulièrement entrâ̂nante. Leur supériorité se manifeste à première vue par leurs traits plus intelligents, leur force musculaire, leur habileté à des travaux spéciaux, leur expérience. Ce sont d'ailleurs des qualités individuelles et non des origines plus nobles qui leur confèrent cette espèce de primauté. Tels quels, ils sont d'excellents auxiliaires, d'humeur très stable, car plusieurs des capitas de la Princesse Clémentine étaient depuis plusieurs années attachés au service de la navigation sur le Congo. Les manouvres les plus délicates sont exécutées par des indigènes originaux du haut fleuve. Les Bangala notam- 
ment ont des dispositions innées pour la marine. Un barreur noir, après quelques voyages, conduit avec la plus grande précision un bateau à travers les méandres du Congo. L'œil, une mémoire surprenante des sites lui tiennent lieu de carte hydrographique. Le capitaine de la Princesse Clémentine, M. von Oertzen, un routier des océans, rendait volontiers hommage à cette supériorité innée, presque instinctive. N'empêche qu'étant seul à avoir le sentiment de la responsabilité, le plus difficile à inculquer au "sauvage ", il se tenait à son poste de l'aurore jusqu'au crépuscule, la main sur l'appareil de commande de la machine.

Leslongues journées de navigation, quoi qu'on puisse penser, sont assez mêlées d'impressions variées, d'incidents pittoresques, pour ne point entraîner de monotonie. Non pas que des paysages d'un exotisme flamboyant se déroulent sans cesse à la vue. L'eau et le ciel dans des étendues infinies, des horizons tendres, des îles basses émergeant d'une brume transparente, voilà les plus fréquents des spectacles.

Les bouches de l'Escaut, quand très mollement le fleuve quitte la terre de Zélande toute gonflée de sève, les ciels chargés de buées de la Hollande, certains coins du pays anglais pourraient être pris comme termes de comparaison, n'était qu'en Afrique la lumière jaillit et s'épand en ondes infiniment plus irradiantes sur toute la nature, alors même que le soleil se dissimule derrière les nuages. Par les rives encore s'accuse la différence, pour autant qu'on les touche dans les mille circuits que décrit le bateau parmi les chenaux, les archipels, les 
lacs où libre, capricieux et magnifiquement sauvage le Congo s'est fait son lit. Passé le Pool, après le chenal, on longe des collines escarpées, encore teintées de rouille à leur sommet, car la saison sèche poursuit son œuvre de dénudation bien au delà des terres pierreuses des monts de Cristal. Plus en amont le terrain s'abaisse et graduellement la végétation devient plus dense. Une première zone semble limitée par le Kasai. A Kwamouth, se trouverait le dernier baobab, posté là comme le témoin isolé d'un groupe particulier de la flore. A Lukolela, en face la Sanga, commence la grande forêt, entretenue par les influences proprement équatoriales, chaleur et humidité constantes. Son domaine, on le sait, est immense. Par places dominent des parasites, brousse et lianes, qui ont attaqué les arbres, les ont étouffés et comme emmurés de leurs vivantes étreintes. Ailleurs, au contraire, l'orgueilleuse futaie l'a emporté. Elle ne rappelle guère les décors d'opéras où sont démesurément grandis des effets de serre chaude. A distance, prise en masse, elle rappelle singulièrement les lignes mouvantes de nos bois. Il faut se rapprocher pour distinguer les formes inédites, les contours rares à nos yeux : les élaïs, les borassus, toute la famille des palmiers aux troncs annelés, à la couronne largement épanouie, pour ne citer que ces essences entre tant d'autres dont le dessin est moins singularisé qu'on ne se plaît à le croire d'après des visions de rêve. Les feuilles cependant ne frissonnent pas légèrement comme dans nos halliers; elles sont plus raides et figées, de port plus hiératique. Et de ce que les arbres de la "grand sylve" ne connaissent pas le sommeil 


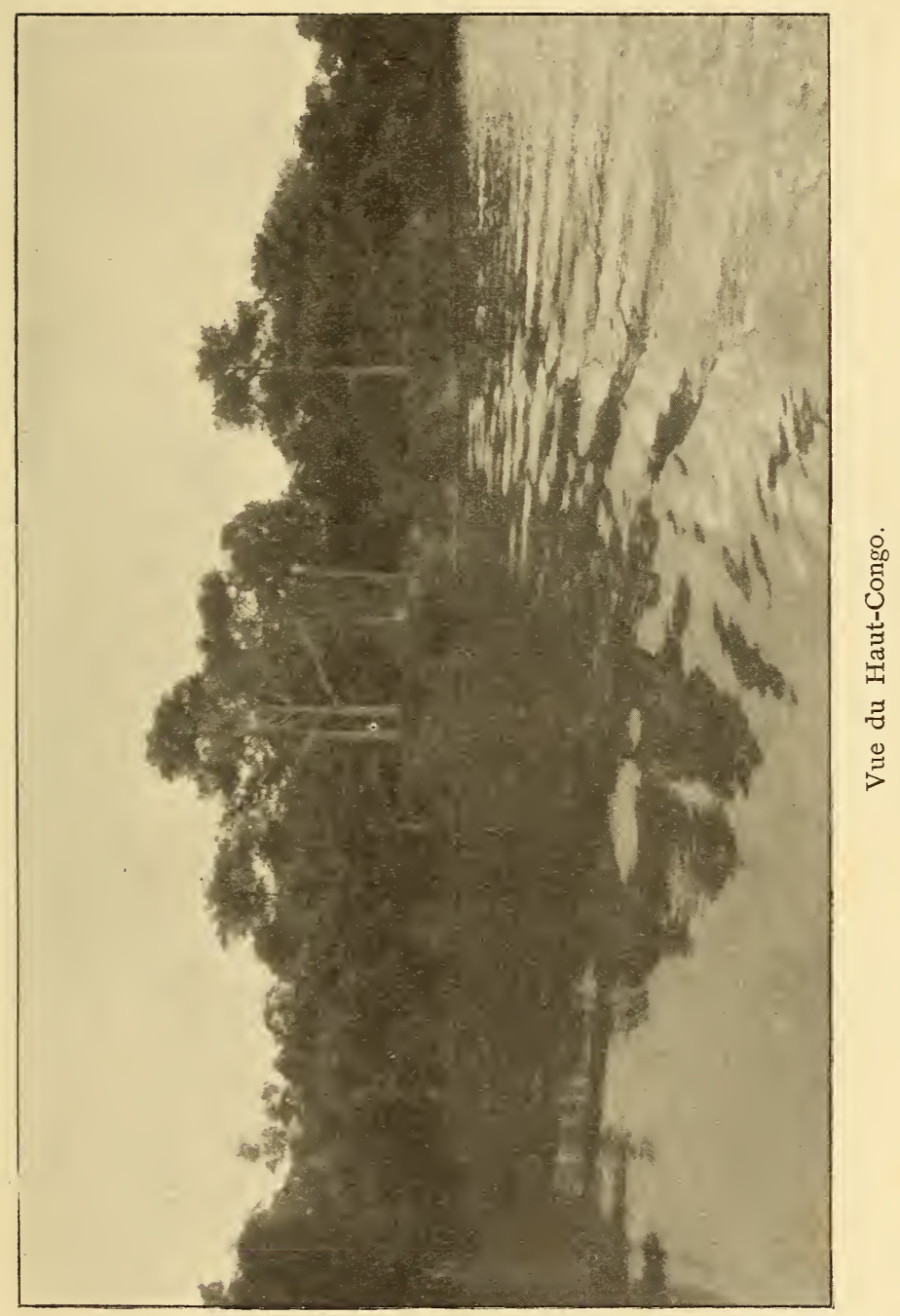



hivernal qui les dépouille de leur parure, ils ne connaissent pas davantage le renouveau délicieux de nos printemps. La nature ne se repose point. Dans la chaleur, dans l'humidité elle poursuit son travail dont les effluves accablent parfois l'habitant du Nord.

Brousse ou futaie, l'une et l'autre opposent un écran impénétrable aux regards du voyageur. Point d'échappées vers l'intérieur, sauf en de très rares occasions. Quand on descend à terre, l'on n'est pas plus avancé. De vagues pistes courent dans un tel encombrement de troncs, de lianes, de plantes épineuses que l'on ne fait que deviner la présence du monde d'êtres qui, à n'en pas douter, pullule dans ces retraites. Croirait-on que jamais je n'ai eu l'occasion, me trouvant dans la forêt, de tirer un coup de fusil sur une forme de gibier, poil ou plume, alors que les perroquets sifflent, qu'on entend autour de soi des froissements de branches et de feuilles. La chasse dans ces conditions est un mythe; il faut connaître les bons endroits, organiser les battues, s'aider des indigènes. Sur le fleuve, pendant la marche, il est loisible de tirer sur les nombreux crocodiles, grands et petits, étendus sur des langues de sable au soleil, ou encore postés sur des troncs d'arbres à moitié déracinés de la berge, d'où ils se jetteront à l'eau à la moindre alerte. Les hippopotames, bien que diminuant beaucoup en nombre, sur le passage des vapeurs, se rencontrent encore par groupes. Un museau à fleur d'eau, une grosse tête levée en sursaut et aussitôt replongée, voilà les seuls buts qu'ils offrent en toute sécurité aux coups de feu. En vérité, pas même au Congo, on ne s'improvise bas-de-cuir. 
Vers le soir, quand le soleil baisse à l'horizon, le bateau s'arrête et va s'amarrer à la rive. A peine l'ancre est-elle assujettie par l'homme qui, moitié nageant, moitié marchant, l'a transportée à la berge, et une planche a-t-elle réuni celle-ci au bateau, que toute la tribu des noirs débarque pour passer la nuit à terre. Presque instantanément les feux s'allument, des tentes, des abris formés de quelques nattes sont dressés; les femmes préparent à manger. Les bûcherons de leur côté vont à la récolte du bois mort ; arbres entiers abattus, brisés ou tués par des tornades et le feu des indigènes. C'est à qui de ces travailleurs se pressera le plus, pour trouver au point le moins éloigné de la rive les troncs et branches dans lesquels il taillera son lot de bûches. Chacun est tenu de rapporter une brasse. Des piquets ont été fichés en terre, à intervalles égaux, pour être remplis à hauteur voulue. Gare! pour celui qui aura boudé à la besogne.

Mais l'obscurité est venue. Dans la clairière, près des grands arbres, flambent les feux de bivouac; des ombres étranges circulent parmi ces lueurs fauves; des coups de cognée retentissent de tous côtés; des groupes plus heureux installés autour des marmites s'échappent des bavardages, des rires, tout un bruit confus et mystérieux Et il en va ainsi de longues heures, jusqu'à l'assoupissement général. Les sentinelles veillent, tandis que dans la nuit montent toujours le battement dailes des grillons, le coassement sonore des grenouilles.

D'accidents, point; mais quelques retards, quand, par exemple, le bateau s'est engagé sur un banc de sable. 
S'il résiste à la manœuvre de la machine en arrière, il ne reste plus qu'un moyen. Tous les hommes vont à l'eau, poussent, tirent, crient, s'agitent sur les commandements rythmés que donnent les capitas, auxquels répondent en chœur les travailleurs. Cela dure une heure, deux heures, une demi-journée, selon les cas. Le capitaine anime son monde de la parole et du geste; les noirs sont enfin excités. Un mouvement se produit, un second, le bateau est de nouveau à flot, il peut reprendre sa marche en avant.

Le I 5 août, à notre arrivée à Stanleyville, notre voyage eut, pour la deuxième fois, comme un aboutissement. Déjà ressentie à Banana, après la traversée de mer, cette impression se renouvelait après vingt-six jours de navigation sur le haut Congo. Le voilà interrompu dans son cours, le fleuve immense qui, à la durée, emprunte des proportions plus démesurées encore. La sensation d'arrêt n'est pas seulement produite par la barrière d'écume bouillonnante, la septième cataracte, devinée presque avant d'être aperçue, mais par la forme aussi des rives. Resserrées, quand on les compare aux expansions d'aspect lacustre auxquelles elles se prêtent en aval, elles s'infléchissent ici selon une courbe qui, appuyée d'une île, ferme en apparence toute issue. De plus près l'illusion s'évanouit, tandis que l'obstacle a grandi. On l'entend et on la voit, la chute qui, déferlant sur les rochers, jette à l'écho son éternelle chanson.

Au moment où nous approchons de la rive, le soleil de l'après-midi brille dans un ciel semé de nuages 
floconneux. Des arbres, au panache sombre, en forme de parasol, projettent de-ci de-là leur ombre légère, en arrière de la berge, laissant à découvert, parmi leur ramure espacée, les habituelles tâches blanches ou grises, ou rougeoyantes des toits et des maisons de la station. A bord les tamtams font rage; les sondeurs placés à la proue du bateau plongent avec plus d'ardeur leurs longues gaules dans le courant, pour les agiter vivement en l'air, après chaque coup de sonde. Tout le petit peuple qui vit sur le pont inférieur pousse des cris, se démène, exultant de joie. Pourquoi? Parmi ces 'noirs, point ou peu de passagers. Gens d'équipage ou autres, la plupart vont sans but. Mais, à leur manière, ils expriment ce sentiment inné de bien-être que procure un long trajet heureusement parcouru.

A terre on nous a préparé une réception pleine de somptueuse cordialité. Bien que nous n'ayons été signalés qu'à la dernière heure, le rassemblement a été sonné pour la troupe qui, en grande hâte, s'est massée sur le rivage, prête à rendre les honneurs. Saluant le bourgmestre de Bruxelles, la musique militaire joue la $\mathrm{Bra}$ bançonne, puis la marche d'Ä̈da. Même sans trompettes thébaines, quel triomphe! Au centre de l'Afrique, - et quand on est du cortège, - une griserie se produit en vous qui monte, bienfaisante. On pourrait sourire car le spectacle est bizarre; on est plutôt un peu ému.

La scène s'est encore animée du concours empressé des habitants : d'une part les blancs à l'allure militaire, d'autre part les noirs, chœur bruyant et mobile en son insouciance. Le bateau " qui monte " c'est pour ceux- 
là le courrier espéré, attendu avec impatience; pour ceux-ci c'est simplement l'objet de la curiosité du moment qui leur tient lieu d'occupation. Et la juxtaposition des deux éléments donne, par l'originalité de ses contrastes, un tableau dont le pittoresque savoureux est goûté, aussi souvent qu'il se représente.

Nous sommes redevables de ce bel accueil à M. Malfeyt, le commissaire général du district qui, le premier, nous souhaite la bienvenue. Près de lui se montrent d'autres figures amies de compatriotes, d'autres mains se tendent. Un courant de sympathie est établi entre les nouveaux arrivés et les résidents de Stanleyville. 



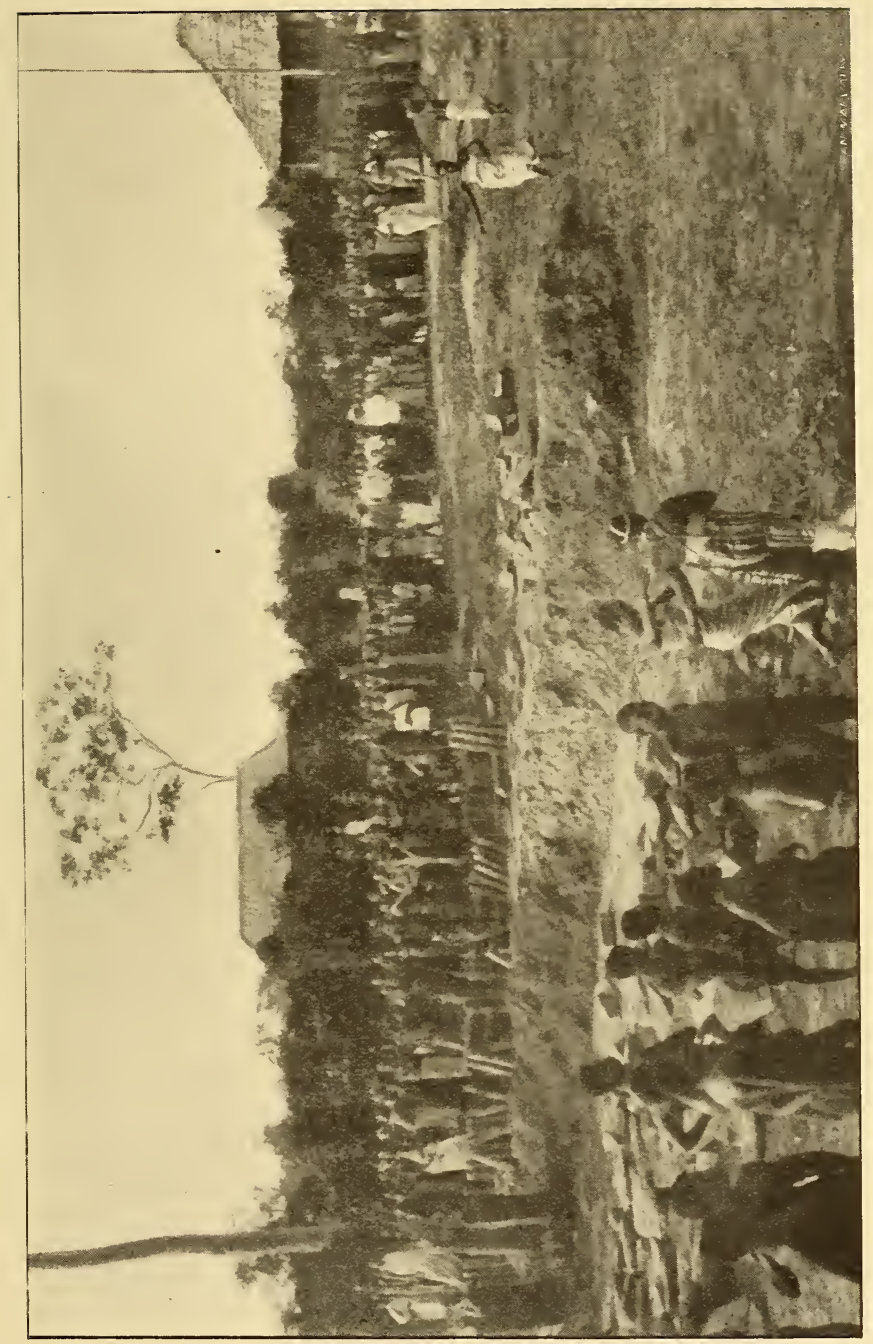

兽 



\section{VI}

\section{AUTOUR DES FALLS}

Stanleyville est le chef-lieu d'un district qui comprenait jusqu'en dernier lieu tous les territoires qui, de l'est à l'ouest, s'étendent du Tanganika au Lomami, et du nord au sud, de l'Aruwimi et de l'Uele aux sources du Congo.

Cette immense province a été tout récemment fractionnée en plusieurs zones. L'ancienne station des Falls, qu'illustra le passage de Stanley, que le fameux TipoTip éleva au rang d'un des grands centres de la puissance arabe dans l'Afrique équatoriale, a été dénommée Stanleyville pour ne plus commander qu'une de ces zones, réduite en étendue et en population. Prise en soi, 
la cité naissante a peut-être moins d'aspect que telle ou telle de ses sœurs établie sur le fleuve. Elle n'a pas la belle ordonnance de Coquilhatville, les constructions importantes déjà de celle-ci ou de Nouvelle-Anvers, le décor de Bazoko, avec son mur crénelé et sa tour arabe, l'aspect imposant de régularité et de méthode des camps militaires d'Irebu, de Yumbi.

Et cependant l'impression que l'on conserve de Stanleyville est excellente à tous égards. Il suffit pour cela de rapporter à ce point central le souvenir des choses vues et observées durant quelques jours seulement.

Dès après Isangi, située à l'embouchure du Lomami, la population se montre d'humeur particulièrement accueillante. Partout où aborde la Princesse Clémentine, pour se ravitailler en bois ou en vivres, les indigènes accourent chantant et dansant, scènes fréquemment répétées, car nous ne doublons pas moins de quatorze villages ou postes durant la journée et demie de navigation qui sépare Isangi des Falls.

A quelques heures du chef-lieu nous avons eu l'occasion de nous arrêter à la Romée, l'importante station agricole établie par l'État sur la rive droite du Congo, en face l'ancien camp qui, sur la rive gauche, était des tiné à tenir les Arabes en respect. Les travaux de la paix ont succédé ici aux dures alertes de guerre de jadis. Un agronome instruit et actif, M. Ambroise, a remplacé les chefs militaires. Les Arabes, où plutôt les nègres arabisés, vivent paisiblement dans leurs villages, cultivant et acceptant de servir d'intermédiaires entre les blancs et les "sauvages " pour amener ceux-ci à faire des planta- 
tions de café. Leur situation est sans doute amoindrie par rapport à ce qu'elle était du temps de leur puissance. Mais s'ils quittaient le pays, elle deviendrait pire, même pour ceux qui peuvent se réclamer d'une authentique origine zanzibarite. On a mis fin à leurs rapines et à leur oppression qui étaient un mal intolérable. On a utilisé leurs qualités acquises de tenue, d'ordre, de dignité, de commandement, qui sont autant de gains certains sur la condition inférieure des autochtones. Le danger qui, le cas échéant, résulterait de leur reste d'influence, serait la propagande musulmane, dont les effets ultimes sont bien rarement en harmonie avec les exigences de notre civilisation. Il ne nous est pas revenu qu'elle s'exerçât d'une façon sensible.

L'action religieuse, au point de vue chrétien, n'est pas négligée dans la zone. A Yakusu est établie une mission anglaise, et tout près de Stanleyville il y a la mission catholique de Saint-Gabriel, de fondation récente.

Quand nous aurons ajouté que des factoreries, soit de la Société d'Agriculture et de Plantation au Congo (A. P. C.), soit de la Société anonyme belge (S. A. B.), poursuivent leurs opérations dans la région, nous aurons saisi les intérêts essentiels d'ordre moral ou matériel qui se groupent autour du centre commun.

Stanleyville rayonne bien au delà du périmètre de la station même, et ce résultat est plus important, plus colonisateur, si l'on peut dire ainsi, qu'une prospérité locale trop condensée et, par là même, un peu artificielle parfois. Sa position lui faisait un devoir de regar- 
der constamment au loin. Un rôle politique et économique des plus essentiels lui était dévolu. Il signifie aujourd'hui la réduction de la domination arabe, la prise en tutelle de la population noire naguère asservie. Ainsi que nous l'avons vu, il ne peut être question de détruire la première force; il faut au contraire savoir l'employer, la ménager. Mais en même temps il faut la surveiller sans cesse, prévenir toute velléité, sinon de révolte, devenue bien difficile dans les circonstances présentes, mais de résistance et de défiance. Les ramifications entre arabisés vont très loin; la circonspection à leur égard est d'autant plus de règle qu'ils affectent extérieurement une soumission plus parfaite, plus entourée de marques de respect et de politesse raffinée.

Stanleyville est encore un centre de ravitaillement, par voie de transit, un nœud de grandes routes dans deux directions, l'une de celles-ci allant vers le Nil, l'autre vers le Tanganika. Les communications doivent être tenues libres d'un côté comme de l'autre, les convois assurés sur des parcours qui, en prenant Redjaf, par exemple, comme lieu de destination, comportent de soixante-dix à quatre-vingts jours de marche.

A Stanleyville aussi se trouve l'un des premiers postes de réception des nouvelles du nord - comme il en existe déjà en aval aux débouchés de l'Aruwimi et de l'Itimbiri -ainsi que de l'est. C'est en quelque sorte un point sonore des régions les plus mystérieuses de l'Afrique. Nous fûmes ainsi des premiers à apprendre les succès du lieutenant Glorie sur les révoltés du Tanganika, et la chaude affaire de Redjaf où le commandant Hanolet 
repoussa victorieusement une furieuse attaque de nuit des derviches venus de Bôr.

Aux Falls, en résumé, l'on reçoit l'impression d'un tout harmonieux dont les parties sont heureusement équilibrées. Chacun y apparaît à sa place, dans le cadre de son savoir et de son pouvoir, avec un autonomie corrélative à la compétence. Venant d'Europe, on est fondé à croire en l'action non seulement utile - elle l'est forcément en dernière analyse - mais encore bienfaisante et humaine de notre civilisation. Nous avons provoqué l'amélioration immédiate de la condition des indigènes jusque-là les moins favorisés du sort, sans détruire les éléments bons à conserver. Les noirs perdraient à la disparition de notre domination au point de vue même où ils sont capables de se placer : leurs intérêts vitaux.

Ils témoignent de ce fait par la façon dont ils se conduisent dans leurs obligations de sujets politiques de l'État. Du moment que celui-ci leur applique un traitement mesuré, ils acquittent sans regimber, paraît-il, les prestations exigées d'eux; produits de la forêt ou du sol pour les gens de l'intérieur, pêches, transports par eaux pour les riverains. Dans leur attitude en tout cas, seule chose que nous ayons constatée de visu, se manifeste une joie de vivre non équivoque. A ce titre certains épisodes des réceptions qui nous ont été faites par les populations des Falls méritent d'être rapportés. Nous les noterons pour leur valeur documentaire aussi bien que pour leur intérêt de pittoresque originalité.

Par terre et par eau, tantôt sur le fleuve, tantôt dans une île, passant d'une rive à l'autre du Congo, en con- 
tournant les chutes, pour revenir au point de départ de Stanleyville, nous eûmes, en la journée du i 7 août, les impressions peut-être les plus vives de tout notre voyage, celles dont le souvenir reste le plus merveilleusement encadré dans notre mémoire.

Au matin, une grande pirogue, amarrée à la berge, nous attendait. Dans ses lignes essentielles elle rappelait le modèle de toutes les embarcations du fleuve : un arbre évidé avec les deux extrémités effilées et relevées en pointe de semblable façon. Mais par ses dimensions elle devenait un vaisseau au regard des véritables périssoires auxquelles se confient les indigènes, même dans les courants et les tourbillons. Armée d'une douzaine de pagayeurs, moitié à l'un bout, moitié à l'autre, elle était parfaite d'équilibre. Tels étaient les canots de guerre que montaient naguère quatre-vintsg, cent hommes et plus. Les temps sont passés où les flottilles se livraient combat sur le fleuve, où les guerriers, brandissant leurs lances, tirant de l'arc, hérissaient de pointes scintillantes, au-dessus de leurs silhouettes noires, les bords de leurs pirogues bardées de boucliers. La nôtre navigue en paix. Les matelots, en costumes blancs rehaussés de cols bleus, - livrée de la marine, - chantent, en plongeant leurs palettes dans l'eau, les hauts faits du blanc, très probablement. Ils conduisent un grand chef, Boula Matari, appellation qui désigne à la fois l'État et tous ceux en qui s'incarnent son autorité, son prestige, sa force. La ville de Bruxelles, saint Michel terrassant le dragon, n'est-ce point assez?

Nous abordons bientôt, à petite distance de la rive 


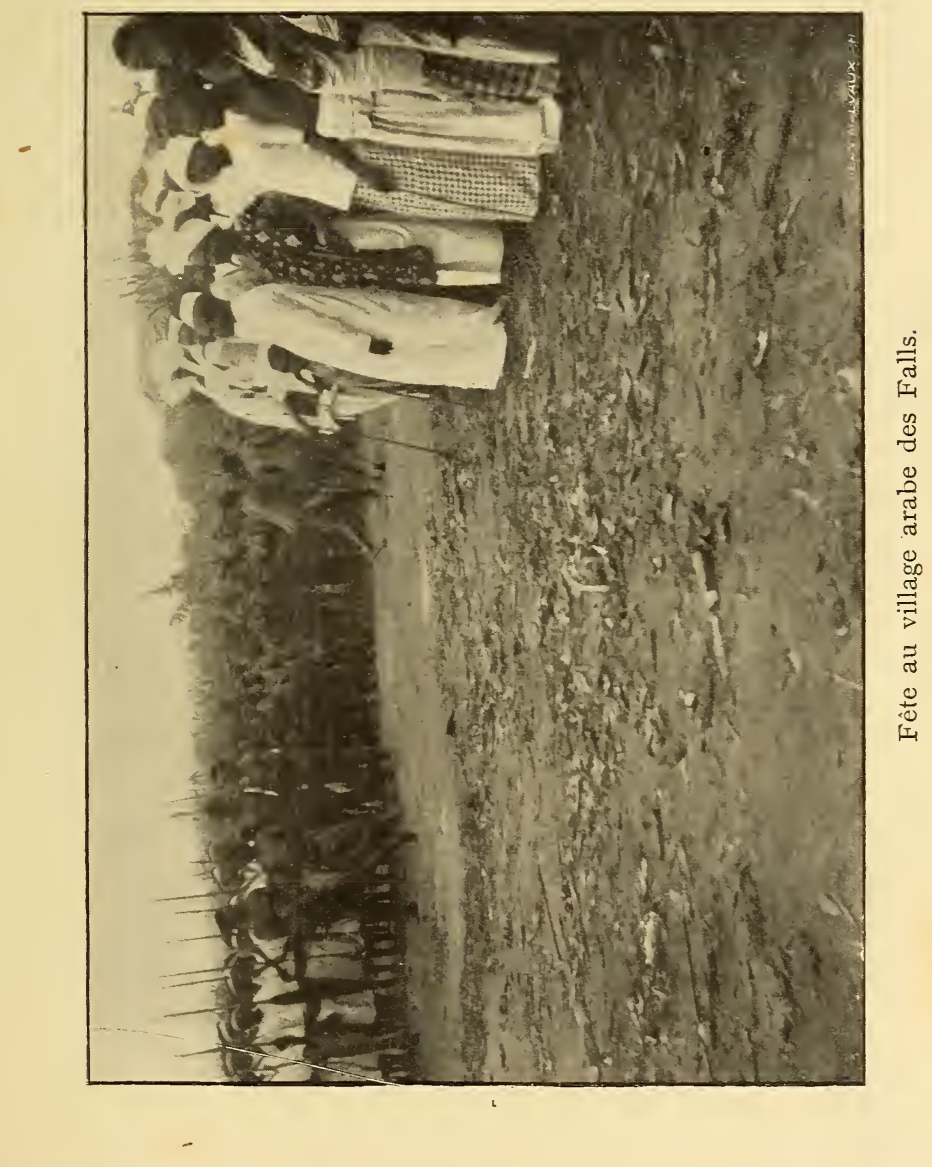



droite, à Kisangani, l'île des Arabes. Ils ont ici une installation qui leur est propre et qui porte, à chaque pas, leur marque. Les habitations ne sont plus des cases ou paillotes, mais bien des maisons construites en bois et en pisé, recouvertes d'un chaume épais et régulier, soigneusement blanchies. Au milieu de la façade, la divisant en deux parties égales, s'ouvre largement la barz $a$, loge rectangulaire qui forme une sorte de véranda en retrait, mieux protégée contre le soleil et les intempéries que ne l'est la terrasse-auvent qui entoure les demeures des blancs. Au fond, une porte ménage l'accès vers l'intérieur; sur les côtés deux fenêtres grillagées; aucune ouverture ne donne vue directement de la façade sur la voie publique.

C'est sur la barza, plus ou moins grande, que l'hôte reçoit les visiteurs, règle ses affaires, tient les palabres.

Son home, ainsi que le quartier des femmes, reste, sauf exception, invisible. L'usage et la politesse, beaucoup plus que les craintes jalouses de l'époux, veulent ici que les femmes ne se montrent pas et surtout ne se mêlent pas à la société des hommes. Aucun type féminin ne requerra donc notre attention; celle-ci sera absorbée par le sexe fort.

Considérable est d'ailleurs la différence que les arabisés présentent avec les noirs qui les entourent. Non pas cependant par la couleur de la peau. La plupart sont des nègres authentiques qui n'ont que très peu - s'ils en possèdent - de sang arabe dans les veines. Venant de la côte orientale ou du Manyema, ils ont simplement subi le contact de Zanzibar. 
Mais l'aspect extérieur a été radicalement modifié. Une ample et longue chemise, d'une éblouissante blancheur, a remplacé le morceau d'étoffe ceignant les reins, ou la quasi-nudité du sauvage primitif. La tête est toujours couverte d'un petit bonnet blanc ou gris, orné d'arabesques brodées, ou d'un foulard de soie noué en formé de turban. Un manteau de couleur foncée, noir ou brun-rouge, soutaché d'or ou de guipures blanches, est porté au-dessus de la chemise par quelques-uns, qui sont évidemment les principaux et les plus élégants.

Dès qu'un de ces Arabes nous aperçoit, il s'approche rapidement, profère à deux, trois reprises la formule sacramentelle de salut, très gutturale à l'oreille, et nous presse les mains entre les siennes, en s'inclinant. Avançant, peu à peu notre cortège se grossit ainsi de tous les curieux qui affluent.

Notre guide est Saidi, chef du village, neveu de Tipo-Tip; il accuse sans conteste, dans ses traits, une origine asiatique : le teint plus clair, la figure plus ovale, la barbe au menton. Assez petit de taille, il en impose moins que tel de ses compagnons; mais l'œil est vif, l'expression du visage intelligente. Il se montre d'une particulière affabilité, plein de prévenances. Au dire de M. Malfeyt, il serait l'un des " ralliés " les plus sincèrement soumis au régime de l'État, désireux d'asseoir son crédit sur l'appui qu'il en reçoit.

Si l'on pensait qu'en d'aussi courtes entrevues, il est impossible de saisir quelque trait de mœurs caractéristique, on se tromperait. A preuve celui-ci que m'a fourni Saidi personnellement. Lui ayant manifesté le désir de 
me procurer un vêtement semblable à celui qu'il portait, il me promit la chose. Le lendemain, au départ des Falls, il monta sur le bateau, ne me parlant de rien. Je crus à un oubli de sa part, quand je fus avisé qu'un paquet avait été mis à bord par ses soins, dans un coin, à l'écart. C'étaient les objets en question. Saidi prétendait m'en faire cadeau, et un cadeau doit se faire discrètement, être trouvé après le départ du donateur. N'est-ce point joli comme raffinement dans la courtoisie? Un donataire bien informé sait du reste à quoi s'en tenir, et il riposte sur le même ton. Barbare de l'Europe, et croyant d'abord n'être qu'acheteur, je n'eus, quant à moi, que le temps de me démunir d'un petit revolver et de le remettre directement, ô confusion! entre les mains de Saidi. Qu'il me le pardonne.

Ces notions d'éducation sont sensibles chez tous ceux qui ont subi des influences arabes. Précisément dans notre suite figuraient encore deux derviches, ou plutôt deux soldats mahdistes faits prisonniers sur le Nil. Leur haute stature, la noblesse de leur maintien, la fierté qui ressortait de tout leur être sont réellement surprenantes. Bagara d'origine, ils appartiennent à la race de ces soldats héroïques autant qu'insensés qui se firent mitrailler, renverser comme des murs vivants, dans la plaine d'Omdurman. Ils devaient être fanatiques, ils devaient nous haïr. Cependant, laissés libres de leurs mouvements dans les environs de Stanleyville, d'où il leur serait d'ailleurs impossible de s'éloigner, sous peine de périr, ils se conduisaient envers nous avec une parfaite urbanité. La veille, dans une promenade au village 
de Mabruki, où ils résident, ils nous firent à la nuit tombante, par un chemin devenu difficile, la conduite en qualité de guides volontaires. De pied sûr, de bras fort ils aidaient ceux d'entre nous dont les pas n'étaient pas suffisamment affermis. En prenant congé de ces braves, si martiaux en leur uniforme de soldats du khalife, si chevaleresques en leur allure, nous ne pensions certes pas à des hommes de race inférieure.

Après la traversée du village arabe, nous arrivons sur une sorte de quadrilatère où toute la population s'est donnée rendez-vous.

La fête commence. Sur l'une des faces sont les noirs, les vrais ceux-ci, non encore teintés de civilisation; leur peau est nue. Hommes, femmes et enfants courent pêlemêle. Ils chantent, ils dansent, ou, si l'on aime mieux, ils crient et sautent. La plupart du temps ce monde trépigne sur place, battant des mains en des rythmes syncopés, faisant des mouvements de hanches et de ventre. Parfois, comme en un quadrille burlesque, un coryphée se détache, homme ou femme, exécute un pas de cavalier seul et rentre dans le rang. Les petiots font chorus avec les grands; des marmots installés sur la hanche de leurs mamans tressautent comme des ballots, leur petite tête moutonneuse tout effarée, mais non inquiète, de ce mouvement et de ce vacarme. Sur la deuxième face du carré les matelots wagenia regardent avec une visible satisfaction, tandis que, leur faisant vis-à-vis, les Arabes ne se départissent pas de leur silencieuse gravité. Pour nous, les spectateurs blancs, adossés aux maisons qui ferment la place, nous sommes 
tout yeux, pour choisir nos points de vue et... photographier.

Ce fut bien autre chose encore quand nous nous remîmes en marche et que, nous précédant, nous suivant, nous frôlant, danseurs et chanteurs continuèrent leurs gambades et leur musique endiablée. Tel le roi David, le bourgmestre de Bruxelles s'avançait précédé d'un peuple en délire, et, de cette foule exubérante, soulevant la poussière sous ses foulées, aux corps ruisselants, montaient des relents de fête sauvage, de kermesse préhistorique... l'Afrique s'amusait.

Autour des chutes mêmes de Stanley, Kizikitini, selon l'harmonie imitative des indigènes, il n'y a pas moins de onze villages de pêcheurs agglomérés. Ils vivent de la prébende du fleuve, particulièrement riche en poissons, là où les eaux se précipitent en cataractes. Des estacades légères barrent le sommet de celles-ci presque sur la largeur entière du Congo. Là les noirs, très habiles, posent leurs nasses; et c'est miracle de voir une pirogue minuscule, conduite par deux ou trois pagayeurs, s'engager dans les vagues et les remous pour aller s'emparer de la proie capturée.

Jusque sur les bords de l'eau, même sur un petit banc de sable, placé près de la rive, gagné a gué par les plus ardents, continuèrent et se renouvelèrent les scènes précédentes. La moins originale ne fut certes pas celle de l'improvisation par un noir d'un chant en l'honneur de Boula Matari. S'accompagnant d'un instrument à cordes composé d'une partie de calebasse sur laquelle sont tendues des fibres végétales, il récitait, sur un 
rythme de 6/8, une sorte de verset. Et le chœur ponctuait la conclusion par un sessa unanime. Qu'est-à-dire ce sessa? Simplement la formule approbative "c'est ça » du français, qu'on nous dit avoir été très familière au commandant Chaltin et avoir été pieusement recueillie et répandue par les noirs. Nous soumettons cette interprétation aux amateurs de grammaire comparée.

Après avoir vu les chutes, dans leur fracas de poussière d'eau, par un temps couvert qui donnait à celles-ci la couleur des vagues de quelque mer du Nord, nous les prîmes par au-dessus, en partant d'un village placé en amont. Voici, non loin de la rive opposée, l'île de TipoTip où s'établit le redoutable trafiquant, où résida, au milieu des embûches et des dangers, notre compatriote Tobback. Souvenir du passé qui fait frissonner et qui fait d'autant plus apprécier la sécurité de l'heure présente. Sur la rive gauche, où nous touchons après une courte traversée du fleuve réduit à de modestes proportions, - l'Escaut à Anvers peut-être, - nous abordons au village d'Inseke dont la population est composée de Wagenia.

Les mêmes démonstrations de joie nous attendent, mais elles se complètent d'un spectacle inédit, celui de luttes entre indigènes, sorte de jeux olympiques. Les Wagenia sont réputés pour leur vigueur physique et surtout pour leur habileté à pagayer dans les eaux tourmentées du haut fleuve. Est-ce par goût, est-ce par nécessité? toujours est-il qu'ils ont été conduits à pratiquer les exercices athlétiques. Notre étonnement n'en fut pas moins grand de voir, chez une population sau- 


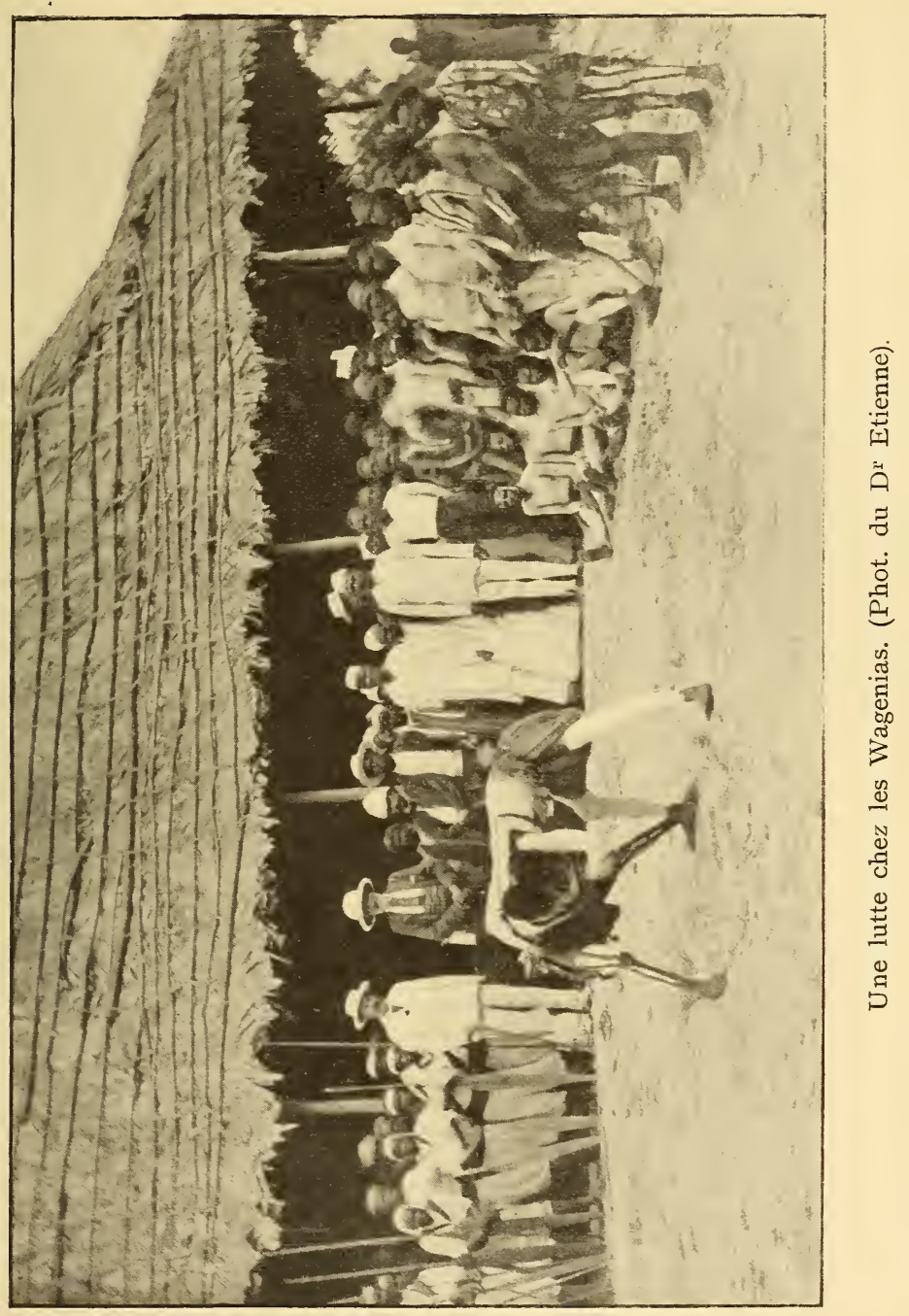



vage, des traditions qui dans une certaine mesure les font ressembler aux héros chantés dans l'Iliade et l'Odyssée.

Toute la population s'intéresse aux luttes. La voilà pittoresquement groupée sur la petite esplanade, qui forme place publique devant la barza diun chef, les hommes debout ou accroupis, au premier plan, plus directement intéressés aux passes qui vont avoir lieu. A l'arrière-plan, les femmes babillant, dansant ensemble. Les lutteurs entrent en lice. Ils sont peints de blanc, de jaune, de rouge, de raies multicolores. Ils sont vêtus d'une ceinture-jupon, à laquelle sont suspendues des queues de jeune panthère, de civette, de putois. Plus il y a de ces appendices, et plus nombreuse ont été les victoires à la lutte dans le passé. Le champ clos est délimité par un homme armé d'une sorte de plumeau, qui, sautant et tournant sur lui-même, feint d'épousseter, de lisser le sol: c'est le féticheur qui écarte les maléfices. Un héraut, un maître, place ensuite les lutteurs corps à corps, avec une minutie extrême, de façon à égaliser les chances. Aussitôt fait, la lutte s'engage; les muscles se tendent, les corps ploient avec une merveilleuse souplesse. Enfin l'un des adversaires tombe, se retire. tandis que le vainqueur défie tout nouveau concurrent. Le chœur des femmes approche, dansant et ihantant, battant des mains, et traverse ainsi deux, trois fois la place.

L'amour-propre des lutteurs paraît très excité. De chaque côté, car il y a toujours deux camps, de petits gamins, pas plus haut que ça, viennent vivement, au moment du combat, recommander au grand - au frère peut-être - de bien se tenir, de l'emporter. 
Tout cela se déroule, selon des rites ou des règles visiblement respectés, avec une grâce, une courtoisie qui donnent un charme et une saveur extraordinaires au spectacle. Qui sait? dans ces corps juvéniles, bien proportionnés, dans cette foule attentive, dans ce groupement harmonieux et naturel, quelque artiste trouverait peut-être le sujet d'un nouveau Ludus pro patria.

Et lorsque nous rentrons de cette excursion, nous nous redemandons encore si la distance qui nous sépare, nous civilisés, de ces grands enfants que sont les noirs, n'est pas due au temps plutôt qu'aux caractères essentiels de l'humaine nature, moins divergents qu'on ne se plaît parfois à le proclamer. 


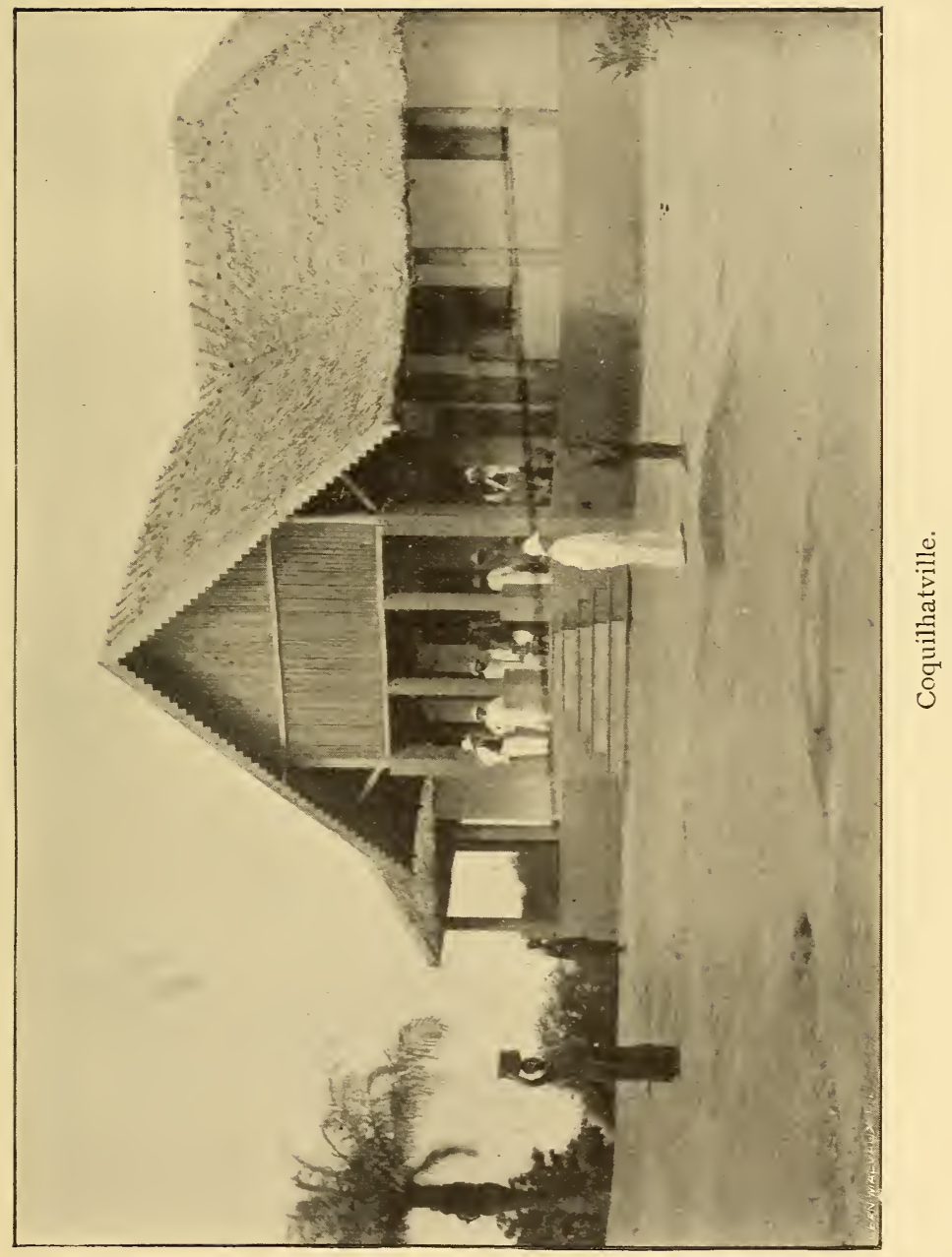





\section{VII}

\section{CENTRES AGRICOLES ET PRODUITS}

La Congolie est relativement peuplée. Même la grande forêt, d'aspect si hirsute et comme inviolé, recèle de nombreux habitants en ses profondeurs. Le voile de mystère dont elle les enveloppe est par eux déchiré en maints endroits. Les hommes sauvages y ont fondé des communautés considérables qui vivent et dans une certaine mesure se développent selon des procédés de travail et dindustrie qui leur sont propres $\mathrm{Du}$ fleuve même sont visibles les plantations des indigènes - les bananeraies notamment - qui couvrent de façon invariable les approches des villages, composés parfois de quelques cases seulement, comprenant ailleurs des 
agglomérations fort étendues, égrenées sur plusieurs lieues de distance.

Cependant une impression toute particulière est ressentie par le voyageur qui, dans ce décor fruste, à peine dégrossi, perçoit les éléments d'une installation méthodique, telle que la conçoivent seuls les civilisés.

Une maison bâtie en briques, qu'est-ce donc en Europe?

Au Congo, c'est la demeure stable, solide, de confort relatif, qui, à elle seule, vaut plus qu'une centaine de cases, abris provisoires, où l'ingéniosité, voire le goût des noirs ne suppléent que dans une faible mesure à l'insuffisance des matériaux et des moyens de construction. Aussi, l'une des premières choses que vous invitera à visiter le chef de poste, comme prenant rang dans les " curiosités " de l'endroit, c'est la briqueterie, avec son hangar de séchage et son four, c'est le tas d'écailles pilées dont se fera la chaux. Et vous admirerez la forme de ces briques, vous les palperez, et vous les ferez sonner les unes contre les autres, pour juger de leur bonne cuisson.

A Coquilhatville, à Nouvelle-Anvers, à Bazoko, après Léopoldville, ce sont des rues, des places, dont les bases durables ont été ainsi jetées. C'est même ce caractère de permanence qui les distingue avantageusement à notre sens, de Boma et plus encore de Matadi, où règne la tôle ondulée, très pratique peut-être, mais toujours marquée, en dépit de ses avantages, d'un faux air de campement. Un coquet et riant village de nos pays a 
bien autre allure. Pour s'en rapprocher de curieuse façon, Nouvelle-Anvers, pour sa part, nous est apparue sons des dehors particulièrement séduisants. Des briques claires ou orangées aux façades, des tuiles - de vraies! - sur le toit, des vaches paissant sur la berge : un tableau de ferme flamande. Non cependant. Des palmiers, aux troncs sveltes et dépourvus de frondaison latérale - des arbres sans membrure - projettent du haut de leur cime qui s'étale une ombre exotique sur hommes et choses...

Le type de la station tracée avec goût et méthode, dans la plus belle des situations, - au confluent du Congo et du Ruki, - à l'abri des inondations, susceptible de se développer dans plusieurs directions, c'est Coquilhatville, chef-lieu du district de l'Équateur. Appelée au plus brillant avenir, elle occupe dès à présent un rang éminent parmi les créations de l'État Indépendant. Tout y respire l'aise et le confort, une organisation de vie en harmonie complète avec les travaux à exécuter et les besoins à satisfaire. De larges allées, soigneusement entretenues et bordées d'arbres, les unes parallèles, les autres perpendiculaires, par rapport au fleuve, divisent le plateau sur lequel est édifiée la cité. Chaque blanc - il y en a huit - possède sa maison, isolée des voisines par un espace libre. Des magasins, des bureaux complètent l'ensemble, que les vues judicieusement ménagées préservent d'heureuse façon du vice de monotonie. Bien qu'une proposition très connue dise que "le temps ne fait rien à l'affaire ", on ne peut que se montrer étonné du court délai qu'il a fallu 
pour s'installer ici à demeure dans des conditions aussi remarquables. Les premiers établissements de l'Équateur datent de 1889 , mais le véritable essor de Coquilhatville commence en $\mathrm{r} 893$. Deux commissaires de district, le commandant Fiévez et le lieutenant Sarrazyn, ont porté cette prospérité au degré où nous l'avons admirée, comme l'admireraient tous ceux qui seraient appelés à en juger par leurs yeux.

La raison d'être d'une station comme Coquilhatville, et comme Nouvelle-Anvers aussi, est double. Chef-lieu de district d'abord Et cela signifie centre de gouvernement et d'administration, avec les rouages essentiels qui font marcher la machine d'État, qui facilitent la pénétration dans l'intérieur, qui assurent le recouvrement des prestations en nature dues par les indigènes. En second lieu, ces deux localités figurent parmi les champs d'expérience des cultures coloniales entreprises en grand par l'État. Nous avons eu l'occasion déjà de parler des plantations de la Romée, dépendant de Stanleyville Nous en avons vu d'autres, ainsi dans le district de Bazoko, à Barumba-Malembe, en pleine forêt d'élaïs, et dans presque chacun des postes du haut fleuve. Il en existe également dans des districts que nous n'avons pas visités, sur l'Ubangi.Uele, dans le Kasai, dans le Bas-Congo, où le Mayumbe surtout donne lieu aux plus belles espérances.

Ce n'est pas leur faire tort, pensons-nous, que d'affirmer l'espèce de primauté exercée actuellement par Coquilhatville au point de vue spécial qui nous occupe. Pendant deux heures, en prenant à travers 300 hec- 
tares environ, nous nous sommes promenés au milieu de caféiers et de cacaoyers. Sur le pourtour de cette vaste étendue, dont certaines parcelles portaient la trace d'un défrichement récent, c'est la forêt vierge qui forme à la fois limite et rideau. Et le contraste est saisissant, quand on s'arrête là, entre cette haute futaie, emmêlée d'un sous-bois terrible, et l'étendue nette où en lignes correctes et géométriques, séparées par le damier des avenues, s'élèvent à des hauteurs variant d'un ou deux pieds à quelques mètres, les précieux arbustes implantés par les soins de l'homme blanc. Ceux-là ont cinq, six ans, ceux-ci, deux, trois, quatre ans. Et très vite l'on arrive à supputer exactement l'âge, tant est régulière la croissance des plants, quand ils sont soumis à de bonnes conditions de développement.

Un guide, d'une compétence aussi entière que complaisante, nous conduisait : M. Louis Gentil, chef de cultures, qui, après les études spéciales faites par lui en Belgique, a complété celles-ci à Kew. Un an de séjour au Congo lui a communiqué un extraordinaire enthousiasme. La foi en la terre, cette caractéristique des paysans de nos campagnes, s'empare de pareille façon, semble-t-il, du colon défricheur et lui inspire une ardeur dont n'est peut-être pas animé au même point le colon commerçant.

Et que dire du commandant Fiévez encore, qui, aux Bangala, où il continue les traditions inaugurées par lui à l'Équateur, se meut comme une tempête d'activité. En voici un exemple topique. Du 3 au 25 août, c'est-à-dire pendant les trois semaines que dura notre voyage de 
Nouvelle-Anvers à Stanleyville, aller et retour, le commandant avait fait mettre vingtet un mille nouveaux caféiers en pépinière. Et il s'en occupait personnellement, je vous en réponds, en sa qualité de fils de la terre qu'il aime à rappeler.

Est-ce la satisfaction de voir lever ce qu'il a semé, de pouvoir juger jour par jour des progrès réalisés, le fait est que l'agriculteur paraît spécialement adonné à sa tâche et c'est là un très grand bien. Ce n'est pas le seul. Par les moyens qu'il met en œuvre, et en dehors des bénéfices immédiats qu'il peut avoir en vue, il exerce une action économique dont nous voudrions souligner quelques effets essentiels, au point de vue colonial.

La culture a comme premier résultat, en fixant les hommes au sol, de substituer ce qu'on a appelé la mise en valeur intensive à la mise en valeur extensive. Les produits naturels, qu'ils se rattachent à la faune ou à la flore, sont toujours dispersés. La chasse, la cueillette, pour être pratiquées avec fruit, exigent d'énormes espaces, l'isolement relatif des exploitants. La culture les rassemble, les fait vivre en société et, à la faveur des progrès industriels qu'entraîne la réunion des forces physiques et intellectuelles, conduit sûrement à un stade supérieur de civilisation.

Les noirs du Congo cultivent, mais dans une mesure restée insuffisante. Ils n'ont en vue que la satisfaction de leurs besoins immédiats, au prix du moindre effort, et sans souci de l'avenir. C'est ainsi qu'outre leurs bananeraies, qui poussent presque sans requérir de soins, ils n'ont guère que des plantations de manioc. 


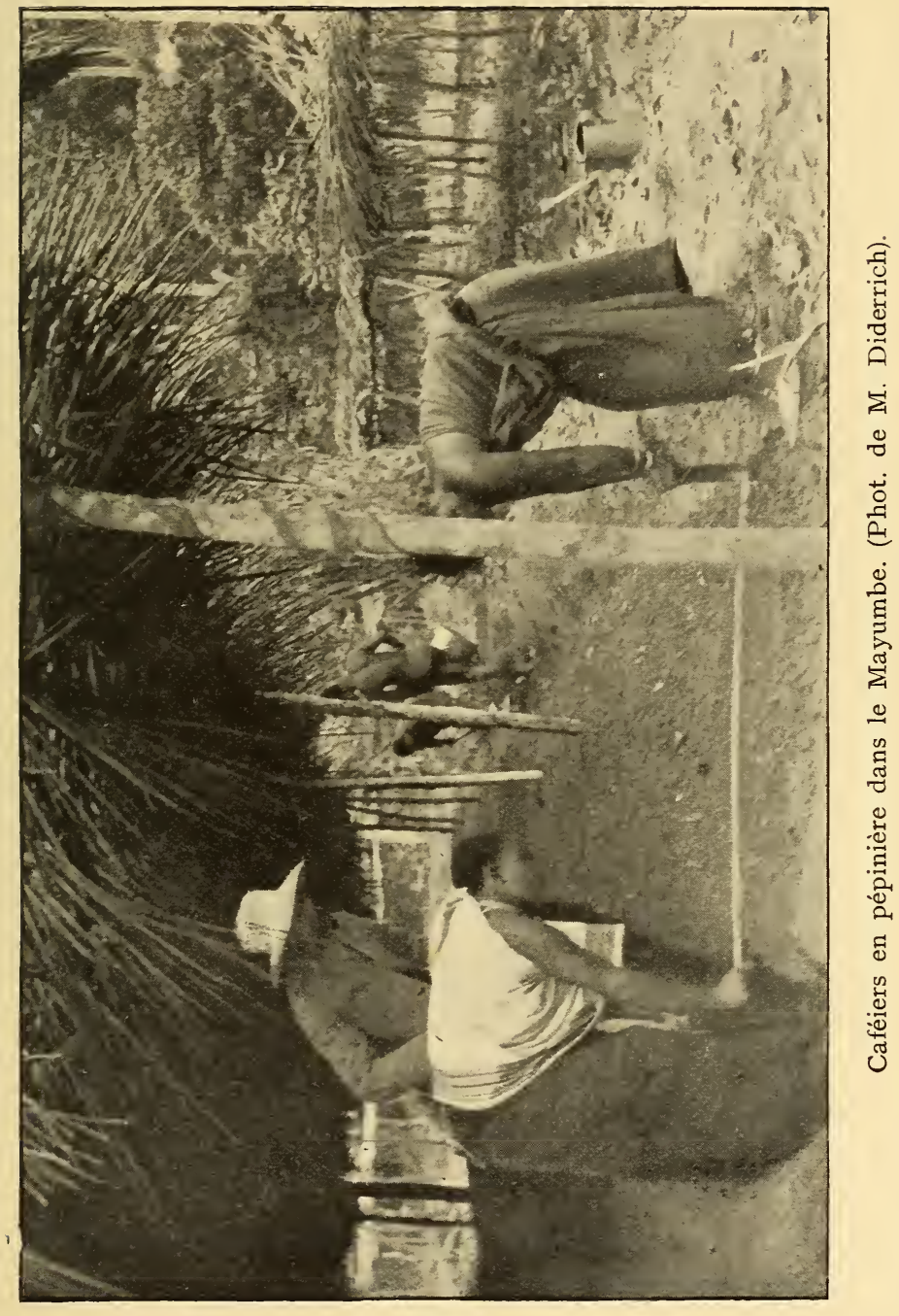



Sans doute il ne faut pas médire de ce produit qui assure l'existence de millions d'êtres humains. Il est d'une venue exraordinairement facile, donne un rendement très supérieur à celui de nos céréales ou à celui de nos pommes de terre. Il est nourrissant et se prête à de multiples préparations, dont le tapioca, chez nous, est l'une des formes bien connues. Mais le manioc présente de sérieux inconvénients. Sa culture se fait en forêt préalablement défrichée et a bientôt fait d'épuiser complètement le sol. C'est, si l'on peut dire ainsi, une culture nomade qui exige des déplacements fréquents. Elle provoque des déboisements inconsidérés et enfin, à raison des facilités qu'elle offre, demande un effort trop minime des indigènes, deux mois de travail par an, nous assure-t-on.

La question s'est donc posée s'il n'importait pas de favoriser d'autres cultures, comme celles du riz, du maïs, du mil, du sorgho, de l'orge. Elles existent d'ailleurs déjà dans la partie savane de l'État Indépendant du Congo, et les Arabes de l'Est les ont même conduites sur une grande échelle. Cependant elles sont entourées d'aléas considérables. Elles demandent une main-d'œuvre nombreuse et relativement experte. Les Arabes se la procuraient par la traite, ne regardant qu'à constituer de grands centres prospères au milieu des régions que systématiquement ils rançonnaient et dévastaient. A défaut de cette concentration et de ces procédés barbares, en fin de compte infiniment plus nuisibles qu'utiles, il serait extraordinairement difficile d'amener les indigènes à créer de nouvelles cultures. 
Pour leur compte ils y répugnent, car il leur faudrait travailler davantage, et quant aux Européens qui les voudraient entreprendre, ils seraient vite arrêtés par la circonstance qu'elles ne paient pas les efforts faits. Sans parler d'une exportation reconnue impossible, qui achèterait les produits à un prix rémunérateur?

Exception sera faite pour la production et la consommation locales, dans un rayon limité, comme c'est le cas pour la culture potagère. Il ne s'agirait plus que de pourvoir aux besoins de petites communautés déterminées, celles qui sont sous la direction immédiate des blancs. Ce côté de la question n'est pas à négliger au surplus. Il est certain que la vie humaine ne saurait être taxée assez haut en Afrique, qu'une bonne alimentation notamment doit faire partie des préoccupations essentielles de l'existence quotidienne chez tous et chacun. Pour résister au climat, à la maladie, à la fatigue, il n'est encore rien de tel qu'un régime substantiel. Les ravitaillements de la métropole, aussi bien exécutés qu'ils soient, - et à pareille distance des bases, ils donnent forcément lieu à des mécomptes, - ne parent pas au déficit possible et probable. Les ressources locales doivent suppléer à cette insuffisance éventuelle. Dans beaucoup de postes et de stations les agents s'en tirent par leur ingéniosité et leur esprit d'initiative. Chacun ne possède cependant pas les connaissances requises. Les tâches à remplir sont multiples, le temps vient à manquer. Des mesures d'ensemble répondront seules aux nécessités croissantes qui sont la conséquence de l'affluence graduelle des Européens. Le potager avec ses 
extensions en cultures de céréales et de tubercules, l'élève du petit bétail et du gros bétail, tout cela rentre dans le problème de l'alimentation dont on se fait malaisément une idée quand on n'est pas sur les lieux.Quoi? un pays quatre-vingts fois grand comme la Belgique, d'une fertilité évidente, peuplé de i 5, 20 ou 30 millions d'habitants et quinze cents blancs n'y trouveraient que difficilement leur subsistance, trois mille, dans les conditions actuelles, y périraient peut-être de faim! Eh bien, non, cela n'est pas extraordinaire du tout. Qu'importent les richesses latentes, si le milieu n'est pas adapté à de nouvelles exigences, si grâce à leurs habitudes séculaires d'insouciance et d'imprévoyance les indigènes ne possèdent ni réserves ni économies. Qu'on veuille considérer les progrès réalisés dans les parties les plus accessibles du Congo, dans le Bas, par exemple, où prospèrent des milliers de têtes de bétail, ou encore dans maint poste du haut fleuve, où la situation matérielle est à tous égards excellente, et l'on sera convaincu qu'avec le temps et l'application des mesures dès à présent décidées, les vices actuels ne tarderont pas à disparaître. Européens comme Africains sont trop intéressés à la chose.

Considérée dans son ensemble, la question tout entière est dominée par une raison économique : les cultures créées ou à créer doivent avoir une valeur de rapport. C'est pourquoi l'on a arrêté son choix sur le café, sur le cacao, de préférence au coton, à la canne au sucre, qui ne sont pas qualifiés pour affronter la concurrence sur les marchés internationaux. Quoi qu'on ait fait dans 
cette voie, l'on ne compte encore que sur les bénéfices à venir. Les expériences instituées à raison des sacrifices de premier établissement qu'elles impliquent, n'avaient pas en vue le gain immédiat, cela va de soi. Elles ont coûté, il n'en pouvait être autrement.

Jusqu'ici les produits bruts du sol sont d'un rendement bien supérieur Avec l'ivoire, qui de plus en plus passe au second plan, il y a en ordre principal le caoutchouc, si répandu dans toute la région équatoriale. Son utilité est d'enrichir à la fois l'exploitant européen et l'indigène. C'est avec le profit que le premier en retire quil peut entreprendre des travaux utiles, livrer le cas échéant au second les articles de ses manufactures. L'effort initial s'est donc porté légitimement de ce côté. Ce fut même la chance heureuse de l'État Indépendant de récolter d'emblée un produit rémunérateur, d'exploiter une mine végétale, qui vaut jusqu'à un certain point les métaux qu'on extrait du sous-sol dans d'autres colonies. Aussi faut-il considérer la fin, avant de condamner à la légère l'action de l'Européen imposent à l'indigène l'obligation de faire circuler la richesse recélée dans les forêts. Il doit la récolter, il doit l'apporter. Impôt ou corvée, prestation en nature, appelez comme vous l'entendez cette charge, en principe elle n'a rien d'abusif. Il n'y a excès que si l'on exige trop de l'indigène. La limite de la modération nécessaire est d'ailleurs vite atteinte, c'est certain. Il convient d'oublier complètement les poids et mesures de la vieille Europe. La contrainte, pour ferme qu'elle soit, doit être entourée de beaucoup de ménagements et d'infiniment de prudence. Le tact politique et 
le désintéressement personnel inspireront sans nul doute la conduite la plus sage et en fin de compte la plus fructueuse. A cet égard la rémunération du travail fourni intervient comme un tempérament utile au travail obligatoire. Une autre limite qu'il importe absolument de ne point dépasser, c'est la faculté de reproduction du végétal exploité. La destruction de la liane à caoutchouc est inévitable, si, à bref délai, des mesures énergiques ne sont pas décrétées et surtout observées pour un traitement moins expéditif et pour la replantation d'espèces gummifères, au fur et à mesure des suppressions opérées dans la forêt. Sans qu'il s'agisse de cultures proprement dites, l'on arriverait cependant ainsi à un mode d'exploitation mixte, en ce qu'on contribuerait directement au travail de reconstitution de la nature.

Comme on le voit, c'est d'une combinaison d'éléments variés que sortira le système de mise en valeur le plus judicieux des richesses du sol congolais

En favorisant uniquement l'exploitation du caoutchouc, on n'apprendrait pas grand'chose aux indigènes. Ils savent tous grimper sur un arbre, entailler la liane ou la couper. Ils ne seraient pas plus fixés au sol qu'ils ne le sont actuellement, ce qui est cependant plus désirable au plus haut point. D'autre part, si l'on ne faisait que des cultures vivrières, même en les améliorant par rapport à celles qui existent aujourd'hui, il n'y aurait rien à gagner, et peut-être beaucoup à perdre. Enfin les plantations, au sens colonial de ce mot, c'est-à-dire la culture de certains produits de grande alimentation, mais spéciaux et exotiques, tels le café ou le cacao, ne 
rapporteraient peut-être assez, de longtemps, ni aux Européens ni aux indigènes. On ne peut dès lors les tenter avec chance de succès que dans des conditions privilégiées de main-d'œuvre, de fertilité du sol, de transport à bon marché.

Au demeurant, l'évolution nécessaire vers l'ère de l'agriculture ne s'opérera utilement que si l'on procède avec discernement. Il serait téméraire d'affirmer que les terres vacantes du Congo constituent autant de champs fertiles prêts à être ensemencés et à porter de fécondes moissons.

Une période d'attente doit être traversée, pendant laquelle on ne s'effrayera pas outre mesure des méthodes d'exploitation rudimentaires et même un peu abusives, telles que les permettent les circonstances de fait ou les dispositions des indigènes. L'essentiel est seulement de réserver et de préparer l'avenir. C'est ce que l'État a compris en créant de vastes champs d'expériences qui ont une double valeur, et d'enseignement du travail pour les noirs et d'initiation aux meilleures méthodes de culture pour les planteurs qui sans aucun doute s'établiront plus tard au Congo. Voilà les résultats à envisager dans le présent, et ceux-là ne sauraient être prisés assez haut. Pour notre part, nous avons donc de nos yeux vu des plantations superbes où travaillaient de nombreux contingents d'indigènes. Constatation précieuse en présence de ce fait que la question de la maind'œuvre domine tout le problème colonial dans les pays tropicaux où l'Européen n'assume forcément qu'un rôle de direction. 


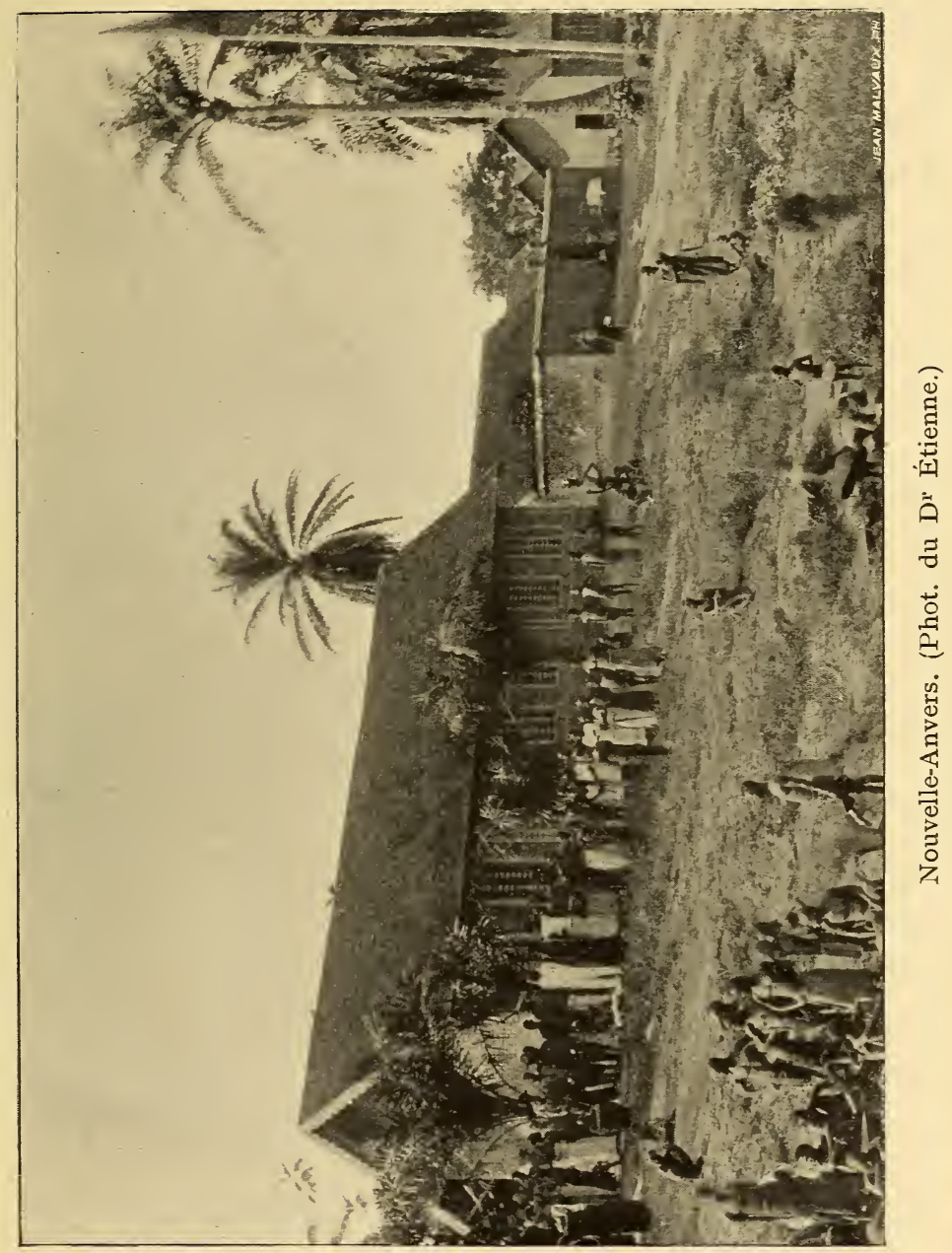



La contrainte, de quelque nom qu'on l'appelle, est à la base du système. Et à vrai dire, elle se concilie avec le sentiment indéfectible de nos devoirs d'humanité. Très visiblement, dans leur corps, comme dans leur constitution mentale, les noirs n'ont pas à souffrir, ou du moins ne subissent aucune diminution physique ou morale du chef du régime qui leur est imposé. Qu’on n'oublie pas que la race comprend des types d'une vigueur et d'une endurance également remarquables, et que d'autre part l'expression " travailler comme un nègre ", qui évoque le souvenir des pires traitements infligés jadis aux esclaves, équivaut au Congo et probablement dans la plupart des autres colonies à fournir la moitié, le quart du travail d'un ouvrier blanc.

Au surplus, nous voici dans un champ de caféiers de Nouvelle-Anvers. Des négresses, jeunes et robustes, exécutent un binage en règle, nettoient le sol, envahi entre les plants par les parasites et les mauvaises herbes. Des surveillantes, des capitas femmes, dirigent la besogne, avec une gravité et une autorité qu'on ne leur soupçonnerait pas. Des petits enfants jouent à côté de leurs mamans; de ces groupes, répartis sur plusieurs hectares, s'élève comme une rumeur d'insouciance, soulignée par maints gestes nonchalants, des pauses fréquentes, des bavardages, des rires mêmes.

Et cependant, c'est le grand chef qui passe, redouté et autoritaire, qui voit tout de l'œil du maître Nos paysans, au temps de la récolte, paraissent autrement plus recrus de fatigue, car eux se pressent, chose qu'au grand jamais aucun noir n'a faite ni même essayée. 
Si le travail est obligatoire, il n'en est pas moins entouré de garanties multiples. L'engagement est à temps, il est soumis à des vérifications minutieuses, à des interventions sévères de la part des représentants de la loi. Enfin il est salarié. En principe, le salaire est représentatif du prix moyen de la subsistance de chacun, bien qu'il laisse le caractère de prestation au travail fourni. En d'autres termes, l'indigène abandonné à luimême ne ferait rien, sauf exception; mais du moment qu'il est astreint au travail, il reçoit une rémunération qui le paie de ses peines. Est-elle suffisante? Oui, si, comme c'est la règle, elle pare à ses besoins en nourriture, en étoffes. Les prolétaires d'Europe pourraient-ils en dire toujours autant?

Le paiement, bien entendu, s'exécutera le plus souvent en nature, et les prix de base subiront beaucoup de fluctuations, car les marchandises de consommation n'ont pas fait l'objet d'échanges généralisés entre régions ou entre communes. Le fil de laiton, sectionné en minces bâtonnets de longueur variable, tend à se généraliser comme monnaie. Son pouvoir d'achat, à mesure qu'on s'avance sur le haut fleuve, s'accroît rapidement Il nous coûte quelques centimes et ces quelques centimes constituent un gros salaire dans beaucoup de stations, car ils permettent de vivre et même d'économiser. La population qui entoure les postes de culture bénéficie dans une mesure très appréciable de la création de ces centres d'activité. Elle contribue à l'alimentation d'une agglomération qui ne vit pas de ses propres ressources, - car les cultures 
vivrières absorberaient tout son temps, - mais qui est occupée à créer des richesses pour la collectivité. Il s'établit donc des relations suivies entre la banlieue parfois à deux, trois jours de marche à l'intérieur — et le centre, que celui-ci soit un chef-lieu administratif, une station de plantation, un camp militaire. Le travail appelle ainsi le travail. La contribution extérieure est également prestée dans les conditions que nous venons d'indiquer.

Les indigènes, à vrai dire, connaissent de longue date les marchés, mais pour l'offre comme pour la demande, ces marchés ne sont pas capables d'entretenir un commerce de vie urbaine. De là, l'obligation conférée par l'autorité aux villages répartis dans un certain rayon de venir livrer aux blancs les produits nécessaires. Il arrive cependant qu'à la contrainte du début, se substitue l'offre d'un apport spontané, quand, par exemple, le noir est friand de l'article qui lui est livré en paiement.

Lorsqu'on considère ces différents aspects de la question du travail, comme il convient de le faire, on admettra que l'indigène, homme ou femme, qui est assujetti à la loi de l'homme blanc, n'est aucunement traité en paria, comme certains l'ont laissé entendre. Il nous est apparu, au contraire, que dans les centres constitués par où nous avons passé, les indigènes sont voués à des conditions normales d'existence, très supérieures, certes, à celles où les laisse leur état originaire de sauvagerie, car là est le criterium obligé.

Avant de s'apitoyer sur la dureté de nos exigences, il 
faut, en bonne logique, se rappeler la situation de l'habitant primitif, du nègre authentique. Or, que voyonsnous? Partout l'esclave qui travaille pour un maître paresseux, et la femme presque toujours traitée en esclave. Le voisin, ou l'ennemi, considéré comme viande de boucherie, dès qu'il est capturé, faute en partie des ressources que procurerait une meilleure discipline de travail. En un mot, dans la plupart des cas une affreuse stagnation morale et physique. Est-il interdit d'espérer que le travail, même imposé, agira comme une cause efficace de relèvement? Il nous a paru que oui par les judicieuses applications relevées sur le terrain. Aussi est-ce dans cette vision réconfortante que nous avons vu à l'horizon s'effacer les silhouettes de Coquilhatville et de Nouvelle-Anvers. 


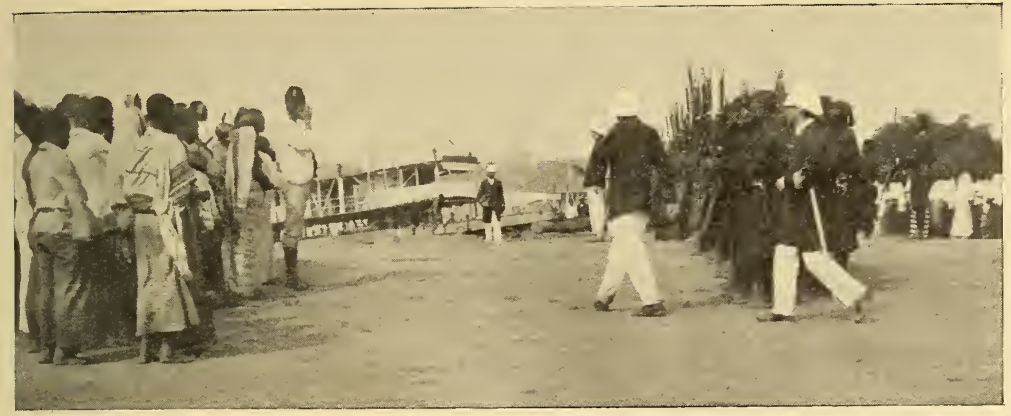

\section{VIII}

\section{LES CAMPS MILITAIRES}

On sait l'importance prise au Congo par les institutions militaires. Elles répondaient à un besoin évident, celui d'assurer l'occupation d'un vaste territoire et d'y faire régner l'ordre et la sécurité Aussi visible que fût le but, il était néanmoins très difficile de l'atteindre. Il fallait, à cette fin, créer des organismes nouveaux, dont aucune épreuve antérieure ne pouvait faire préjuger la valeur. Au Congo, pour la première fois, a été réalisée l'expérience d'une armée indigène recrutée presque exclu- 
sivement sur place, parmi les populations réputées les plus sauvages et, au sens propre du mot, les plus anarchiques qu'on pût rencontrer. Pour encadrer cette masse, quelques rares éléments européens. A l'encontre des pratiques suivies dans d'autres armées coloniales, aucun corps de troupes n'a été formé de soldats blancs venus de la métropole. Toute discussion sur ce point spécial, soit dit en passant, nous paraît d'ailleurs chose oiseuse. L'État Indépendant n'avait pas le choix. Il ne lui était pas loisible de faire appel à des miliciens belges, et l'on ne voit pas davantage dans quelles conditions il eût pu prendre à son service une sorte de légion étrangère. Il a donc eu recours à ses sujets noirs, et l'événement a prouvé - malgré des incidents douloureux et des vicissitudes inutiles à dissimuler - qu'il a été bien inspiré en les employant pour cette tâche délicate, comme pour les autres.

Quiconque voyage au Congo peut saisir par de multiples aspects les caractères de cette organisation. Il y a des soldats à Boma, à Léopoldville, dans les postes du haut fleuve comme dans ceux de l'intérieur. Cependant, en dehors de la capitale, qui centralise les services de la force publique, c'est dans les camps d'instruction que s'offrent les tableaux les plus complets de la vie militaire. Zambi, en aval de Boma, Yumbi, dans le district de Stanley-Pool, Irebu, à l'Équateur, Umangi, aux Bangala, tels sont les noms à relever le long du fleuve.

Il y a dans chaque camp à noter tout d'abord l'installation et nous savons ce que cela signifie en style colonial : défricher, construire, planter, tracer des routes, faire 
mille et un métiers. De toute cette ingénieuse activité les résultats sont là qui frappent d'autant plus que vous apprenez la rapidité avec laquelle ils ont été obtenus.

Arrêtons-nous de préférence à Yumbi et à Irebu qui forment types etqui, dans leurs grandes lignes, répondent à un même plan, à la différence près que Yumbi, plus ancien, est aussi plus étendu et plus peuplé. D'un côté comme de l'autre existe un plateau divisé régulièrement en larges avenues et rappelant par ses aménagements un modèle cher à nos officiers : Beverloo en Campine. Les préoccupations utilitaires viennent toujours en bonne place. Des essais de cultures tropicales, café et cacao, ont été poursuivis dans les camps comme dans les stations exclusivement agricoles. Grâce encore à la maind'œuvre que procure la force publique, il a été possible d'y former des potagers. Le lieutenant Jeuniaux, à Irebu, en vrai soldat-laboureur, ne considérait pas comme le moindre de ses succès d'avoir en abondance des légumes frais des espèces européennes. Les premières pommes de terre poussées à Yumbi, croyez-le bien, ont été un sujet d'admiration.

La population des camps est par définition composée de soldats, ou plutôt, en majorité, d'apprentis soldats. Miliciens et volontaires forment les contingents de la force publique, dont le total s'élevait récemment à environ quatorze mille hommes. Le service actif comporte en principe, pour les miliciens, une durée de sept années, dont deux passées à la réserve. Des dispositions récentes ont créé en outre des corps de réserve qui comprennent à la fois d'anciens soldats libérés du service actif, des 
miliciens et des volontaires; leur rôle rappelle jusqu'à un certain point celui des ressortissants des anciens confins militaires en Autriche-Hongrie, ou encore celui des cosaques. L'obligation militaire gagne en durée, soit douze ans, ce qu'elle perd en intensité. Elle a pour objet de tenir réunis, par une sorte de fraternité d'armes, des éléments qui, dispersés, perdraient toute efficacité. Groupés en villages, ces soldats apporteront, le cas échéant, des renforts considérables à l'État.

Yumbi et Irebu ne rentrent d'ailleurs pas dans cette catégorie. Ce sont des camps d'instruction purement et simplement. L'effectif des troupes était, dans le premier, d'un millier d'hommes, et dans le second, de sept à huit cents hommes environ, à notre passage. Il faut ajouter à ce nombre la population féminine, beaucoup de soldats étant mariés. Par ce seul détail, si l'on peut dire ainsi, on voit que le régime de la caserne, tel qu'il fonctionne en Europe, n'est pas de mise au Congo.

Sans doute, l'individu, des deux côtés, est privé d'une certaine somme de liberté. Au Congo, il sera encore plus déraciné, étant données d'une part les distances, les différences de mœurs et de langage qui départagent des tribus établies sur un territoire grand comme quatrevingts fois la Belgique, et, d'autre part, le désir de l'administration de garder le soldat, même libéré du service actif, dans certains centres, à sa disposition. Mais sans hyperbole aucune, le soldat, congolais, une fois incorporé, n'a pas à médire de son sort. Et de fait il ne se plaint pas. Pour s'en rendre compte, qu'on veuille bien le suivre dans sa vie militaire. Le métier d'abord. Eh 
bien! il n'y a pas de doute possible à ce sujet. Le noir est très fier d'avoir un uniforme, de porter un fusil qui fait de lui un guerrier redoutable et respecté. Si les peuplades du Bas-Congo sont généralement de tempérament mou et poltron, par contre celles de l'intérieur sont parfois très fières et éminemment courageuses. Bangala sur le Congo, Sakara et Azande au nord, Bakusu ou Batetela dans le sud, sont tous dans ce cas. En ce qui concerne les aptitudes physiques, il faut à peu près constater les mêmes différences. Plus on se rapproche de la côte, et plus on perçoit les causes de dégénérescence, l'alcoolisme notamment, dont on ne dira jamais assez les effets néfastes.

Normalement, les noirs sont vigoureux, bien découplés et adroits de mouvement. Aussi ont-ils très vite fait, dès qu'ils ont compris les commandements, de les exécuter à la perfection. Le maniement d'armes, qu'on admirait tant à Tervueren, se fait avec le même brio par toute compagnie quelconque du Congo. Un exercice, dont raffolent les noirs indistinctement, est l'escrime à la baïonnette, qu'ils pratiquent avec la virtuosité qu'ils mettraient à une danse de guerre de leur cru. Et quant au zèle individuel de l'homme, il serait difficile, pensonsnous, d'en trouver un témoignage plus probant que celui d'une sentinelle de la Princesse Clémentine. Chaque soir à peu près, le soldat répétait consciencieusement, pour lui, fusil en main, les commandements réglementaires : "Portez armes! Présentez armes! Reposez armes! Une, deux, une, deux! ") et cela durait tout un temps. Sa joie fut profonde quand l'un de nous, quoique pékin, put lui 
faire exécuter d'autorité les mêmes mouvements, vagues rappels, pour le chef, des fonctions exécutées dans la garde civique de Bruxelles en Brabant.

Ces aptitudes, ces dispositions spéciales permettent même de tirer grandement parti des indigènes comme commandants en sous-ordres. Il faut avoir vu des sousofficiers faire l'instruction pour juger de quoi ils sont capables. L'explication pour la décomposition du mouvement se fait avec volubilité, en langage indigène. Au cours d'un exercice d'ensemble, sur le terrain, un sergent ayant reçu l'ordre de l'officier blanc de faire tenir les hommes plus immobiles, après un mouvement déterminé, s'écria aussitôt : "Avez-vous des fourmis aux pieds, pour bouger comme ça. " La traduction nous fut donnée par le lieutenant Mauroy, commandant le camp de Yumbi, et c'est de lui aussi que nous tenons que jamais en Europe il ne rencontra de sous-officier, ayant au même degré que son sergent-major noir, l'intelligence des règlements, l'autorité et le sens de la discipline. Ces exemples pourraient être indéfiniment multipliés. Si nous en citons quelques-uns, ce n'est au surplus pas pour en conclure qu'une armée de nègres vaille une armée de blancs. Il manquera à la première les ressorts moraux, le sentiment de l'honneur, l'amour du drapeau ou de la patrie qui animeront la seconde. Il lui arrivera très facilement de perdre courage, de lâcher pied, de s'abandonner à une folle panique, encore que les troupes de l'État Indépendant aient souvent fait preuve au feu de très réelles qualités. Reste acquis, et c'est là le premier fait à retenir, que le métier des armes, pris en lui-même, 


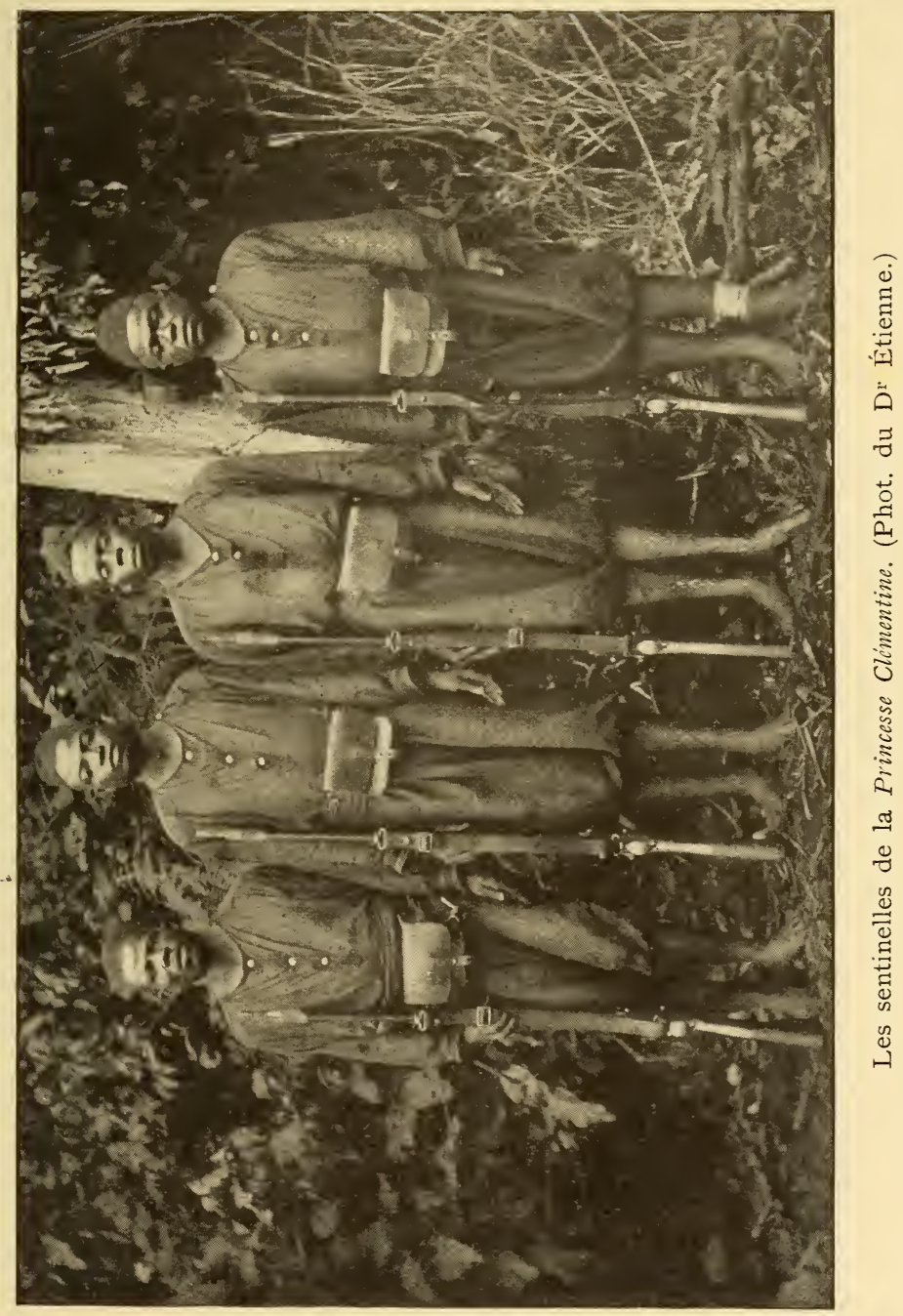



n'inflige pas une diminution physique ou morale à l'indigène. Celui-ci tire au contraire grandement parti de son incorporation dans la force publique à quelque point de vue qu'on se place.

La " préparation à la guerre ", selon la formule européenne, n'est pas l'objet exclusif de l'organisation de la force publique au Congo. Certes, les opérations de la campagne arabe, les expéditions nilotiques, avec les contre-coups qu'elles ont eus en plusieurs parties du territoire de l'État Indépendant, ont nécessité des efforts militaires de grande envergure. Le plus souvent cependant les troupes n'ont à intervenir que comme des forces de police répressive, voire, dans les districts les mieux pacifiés, que comme une gendarmerie d'action préventive. Elles seront les auxiliaires obligés de l'administrateur qui soumet les populations aux devoirs essentiels envers l'État : savoir, le respect de l'ordre et de la sécurité, l'acquittement des prestations exigées par le fisc; les auxiliaires encore des voyageurs qui achèvent la reconnaissance des pays de l'intérieur; des commerçants dont les transactions avec les indigènes ont besoin d'être protégées; des missionnaires dont la propagande entièrement pacifique mérite d'autant plus d'être revêtue d'un caractère d'inviolabilité.

Pour que les soldats noirs soient aptes à remplir ce rôle éminemment social, il importe de leur inculquer des notions de vie civilisée, pour le moins autant que les éléments du métier des armes. C'est une tâche d'éducation qui demande beaucoup de temps et de patience et qui, pour réussir, doit être abordée par plu- 
sieurs côtésà la fois. La recrue noire n'aura pas seulement à se préparer aux devoirs du soldat en campagne, mais encore à s'initier aux conditions d'une existence plus active, plus industrieuse et plus utile à la communauté à laquelle il appartient. Ainsi se trouve justifié un service militaire à long terme qui, en échange du temps exproprié, confère à l'individu des avantages indéniables. La discipline aidant, le soldat congolais apprend à travailler. Terrassiers, maçons, charpentiers, forgerons, mécaniciens sont formés sur place, dans ce milieu spécial, sous la direction d'un Européen ou d'un " homme de la côte ». Leur habileté manuelle n'égale pas celle de nos ouvriers, cela s'entend, mais n'en est pas moins très réelle, après un apprentissage relativement court.

En dehors des capacités professionnelles qui sont sollicitées et développées de cette façon chez les individus d'élite, - le petit nombre, - il y a pour tous un nouveau genre de vie à adopter. On s'est parfois plaint des excès et, pour dire le mot, de la sauvagerie des soldats de la force publique; mais il faut les avoir vus à côté des "bushmen ", les hommes de la brousse, ce que nous pourrions encore appeler les nègres indécrottés, pour juger de la différence qui s'établit entre ceux-là et ceux-ci.

Qu'on ne nous prête pas au moins l'intention de préconiser un vaste système d'encasernemeut. Ce n'est pas le lieu. Il n'y a précisément rien qui ressemble moins à la caserne, selon le type connu, qu'un camp congolais. Pénétrez dans l'une de ces enceintes où sont agglomérés 
quelques centaines d'habitants. Ce sont, dans des alignements corrects, se coupant à angles droits, des groupes de maisons en briques ou en pisé formant des logis distincts. Entrez dans l'une de ces maisons. Si elle appartient à un gradé, elle ne sera occupée - il s'agit d'une ou deux chambres - que par lui, sa femme et... l'enfant. Un lit, muni de couvertures et de la moustiquaire, une chaise, des nattes, quelques ustensiles de ménage garnissent cet intérieur. Au mur des gravures de journaux illustrés, ou plutôt des images, un quelque chose, n'importe quoi! de brillant, de bariolé! L'ensemble est soigné, tenu avec un certain souci de coquetterie. Dans le quartier des hommes, ce sera davantage " chambrée ", mais encore ordre et propreté y règnent-ils, sans qu'il y ait entassement. Et ici vivent également des ménages, à l'abri des promiscuités. Comparez maintenant avec les chimbèques des "sauvages ". Nous ne dirons pas que tout y est laid. Les parois seront, le cas échéant, d'un curieux travail d'ajustage et de vannerie, mais quelques lattes, surhaussées au-dessus du sol, composent le lit, deux ou trois pots pour toute vaisselle gisent à terre. C'est nus ou à peu près que les habitants se blottissent sous le toit très bas et enfumé qui leur sert de pauvre abri.

Vêtus, logés, nourris, les soldats congolais sont traités comme le sont les soldats réguliers en tous pays, avec cet avantage en plus, en leur faveur, que leurs obligations militaires leur procurent une augmentation de bien-être incontestable par rapport à leurs habitudes antérieures.

N'oublions pas la solde qui, fixée à 2 I centimes par 
jour, représente dans le milieu une haute, très haute paie. En effet, à l'Équateur, dans les camps de Yumbi ou d'Irebu, où nous nous reportons, la ration quotidienne d'un homme - la chikwangue assurant sa subsistance - revient à un mitako, soit ce bout de fil de laiton valant, comme monnaie locale, un ou deux sous. Trouvera-t-on exagérée alors l'expression de ce missionnaire anglais qui nous rapportait que les soldats congolais s'estiment des lords, des seigneurs. Dans sa pensée, il est vrai, ce sentiment de l'indigène conduisait, par une pente naturelle, à l'emploi abusif de la force que lui conférait son caractère militaire. Tel l'homme d'armes qui jadis, au service d'un baron féodal, abattait durement, sur le serf corvéable à merci, sa main gantée de fer.

L'observation toutefois se rattache alors à un autre ordre d'idées, celui des méthodes politiques ou administratives. Le rôle de l'élément militaire demande à être strictement délimité et entouré de multiples garanties, chacun peut en convenir. Mais cette considération n'est de nature ni à énerver les qualités intrinsèques d'une institution, ni à détruire les profits directs ou indirects qu'elle est susceptible d'engendrer. La question est toute résolue, pensons-nous, si l'on envisage le sort des soldats congolais en lui-même. Il ne suffira pas, en manière d'objection, de rappeler les mutineries, les rébellions qui se sont produites à différentes reprises, et qui ont été suivies de répressions douloureuses et, en dernier lieu, de véritables campagnes. Il faudrait, chaque fois, analyser, dans leurs tenants et leurs aboutissants, les causes de ces mouvements de révolte. Pour la colonne Leroy, de 
l'expédition Dhanis, - origine du soulèvement d'un groupe considérable de Batetela, - les privations inséparables d'une guerre difficile, l'inexpérience de certains chefs expliquent bien des choses. En station, où le fait est d'ailleurs beaucoup plus rare, croit-on que l'esprit de mécontentement s'élève et souffle au hasard, sans motif particulier, aisé à déterminer? Suivant les cas, n'y a-t-il pas eu malentendu, ordre donné à tort ou faussement interprété, une querelle, futile dans ses origines, mais qui s'est envenimée, une injustice même? Car chacun est faillible, du premier jusqu'au dernier.

L'idée fausse qu'il importe de dissiper, c'est de vouloir attribuer à des causes générales ce qui est uniquement dû à des causes locales, très simples et très ordinaires.

Non, au Congo il n'a jamais été question d'un mouvement " national " contre l'oppresseur étranger, de la réaction de la " conscience indigène ", religieuse ou civile, contre les entreprises de la civilisation occidentale. Ces tentatives sont possibles dans des pays avancés, parmi des Hindous, des Arabes, mais non chez les noirs du Congo. La ration a-t-elle été régulièrement distribuée, la fatigue a-t-elle été proportionnée à la force d'endurance de chacun, la punition, en cas de faute, a-t-elle été équitable et justement appliquée, la femme d'un tel a-t-elle été respectée par un tel autre, voilà, le plus souvent, où portera avec le plus de fruit l'enquête nécessaire. De façon absolue, ce n'est point le régime militaire, "la caserne et la schlague ", suivant la définition de quelques-uns, qui, malgré ses obligations assez dures, répugne au tempérament individuel du noir. 
Il était intéressant de suivre, dans ses effets les plus concrets, l'action des organismes militaires au Congo. En réalité cette action, en dehors même de la force publique où elle se concentre, domine toute l'œuvre congolaise. Il n'est pas de colonie au monde, pensonsnous, où elle se soit manifestée avec une pareille énergie, où elle ait joué un rôle aussi prépondérant, pour ne pas dire exclusif. La vie civile, dans ses diverses manifestations, est régie par les mêmes règles de discipline minutieuse. Le réveil, les repas sont sonnés par les clairons, la retraite aussi; la cloche des travailleurs, c'est encore le clairon. Tout agent va "au rapport " devant son chef hiérarchique, de même que dans une compagnie ou dans un régiment.

Cependant, et voilà peut-être le trait le plus curieux à noter, l'allure militaire imprimée au système entier n'évoque en rien l'image du régime du sabre. Sous l'influence des besognes coloniales qu'il doit remplir, l'officier a évolué. Conducteur d'hommes, il le reste, mais d'une autre façon, puisqu'il administre, qu'il juge, le cas échéant, qu'il préside aux transactions économiques. On peut estimer que ces méthodes ont quelque chose de provisoire, que dans les cadres complets de la future colonie il faut prévoir d'autres répartitions du travail, chacun faisant ce qui lui revient dans les limites de sa compétence spéciale. Il serait profondément injuste toutefois de méconnaître l'énorme labeur accompli par les officiers au service de l'État. En vérité ils ont fait le Congo ce qu'il est, sous les auspices d'un système que nous qualifierons volontiers de régime du clairon. 


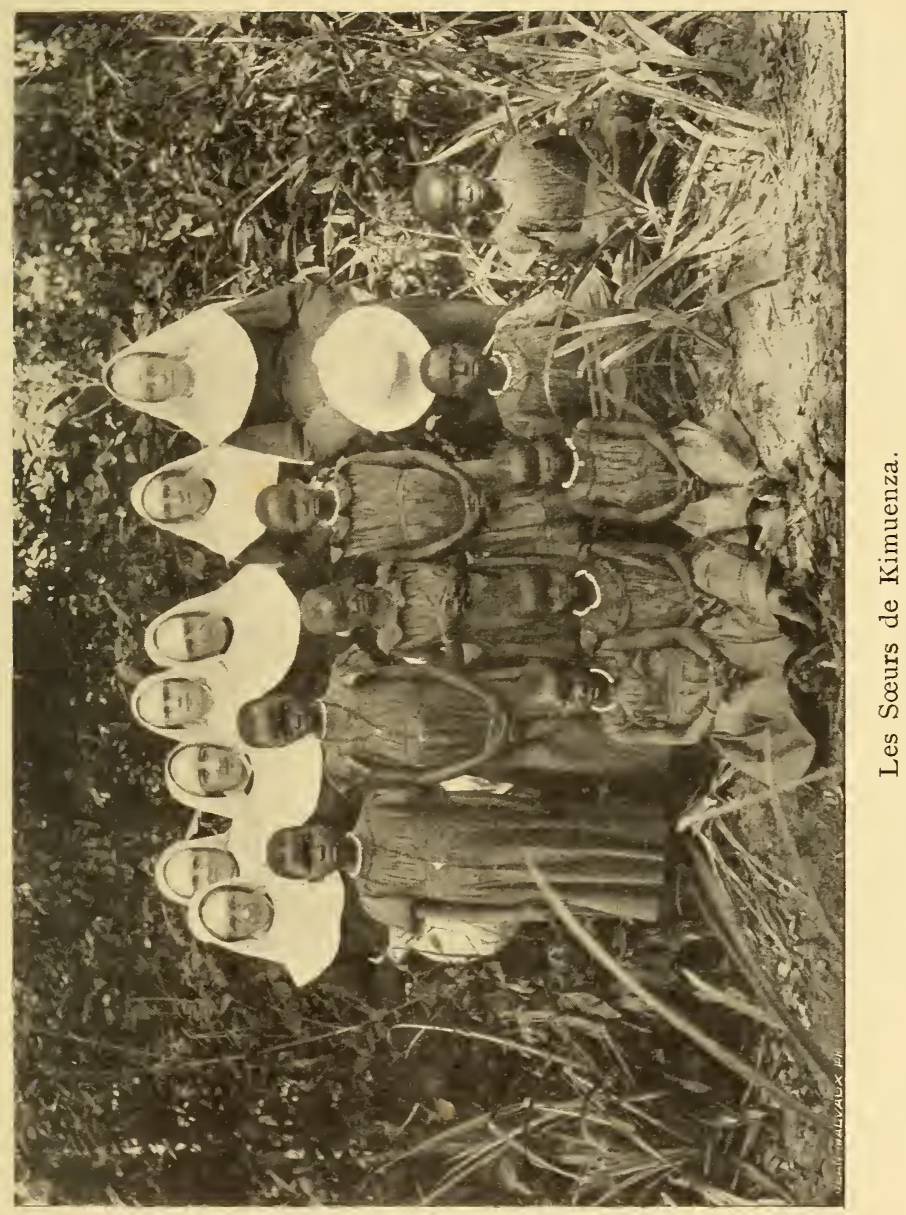





\section{IX}

\section{LES MISSIONS}

Dans la plupart des colonies fondées par les pays de civilisation chrétienne, les missions religieuses ont joué un rôle actif, en rapport intime avec la propagande de caractère universel que suscite la vocation même de leurs membres.

Considérées dans leur individualité nationale, ces diverses communautés représentent si bien une part de l'influence exercée au loin par la puissance à laquelle elles ressortissent respectivement, qu'il n'existe peut-être pas de gouvernement qui, à l'égard des unes ou des autres, ne veille jalousement sur leurs droits et leurs 
intérêts, en favorisant, autant qu'il est possible, leur développement, leur action morale et matérielle.

D'où l'aphorisme, resté célèbre à juste titre, que l'anticléricalisme n'est pas un article d'exportation. Au Congo, en pareille matière, il n'y a aucune difficulté à pratiquer les idées les plus larges et les plus tolérantes. Le terrain n'y est pas disposé pour les conflits de doctrines. La solidarité qui unit étroitement tous les blancs dans la lutte incessante contre la barbarie a raison, ipso facto, des préjugés et des passions, de toutes les intolérances qui rentrent dans le cadre de la civilisation. Les ṕrincipes si libéraux, énoncés en l'acte général de la conférence de Berlin, trouvent dans l'État Indépendant leur intégrale application.

Il n'est apporté aucune entrave à l'exercice de n'importe quel culte; les croyances et convictions individuelles sont entièrement respectées. Unc égale bienveillance préside aux encouragements, aux faveurs, aux concessions qu'accordent les autorités aux représentants des différentes missions. Aussi les établissements religieux essaiment-ils à travers tout le Congo, assurés d'une hospitalité que certains d'entre eux ne rencontreraient sans doute pas ailleurs, en dépit de la lettre des prescriptions internationales. Bien plus, les missionnaires, protestants comme catholiques, ont été associés directement aux mesures prises pour la protection des indigènes. Une place leur est faite dans les commissions spéciales de surveillance et de contrôle instituées à cette fin. Elles ont le droit et le devoir de dénoncer les abus et excès qui viendraient à leur connaissance, remplissant 
ainsi le rôle d'une opinion publique en voie de formation. Rôle qui n'a rien d'illusoire quand on veut réfléchir à la publicité immense que notamment Anglais et Américains trouvent, pour les actes de leur propagande, dans leur presse mondiale.

Et tout cela est juste et mérite d'être loué.

Les missions incarnent une pensée essentielle de désintéressement, indispensable dans un milieu qui, par définition, doit être transformé en un centre d'exploitation.

Loin de nous la pensée de blâmer les mobiles de gain et de spéculation qui sont à la base des entreprises humaines et auxquels la colonisation, en particulier, doit tant d'efforts dignes d'admiration. Mais à ce qu'il y a souvent d'un peu âpre dans la poursuite du progrès économique, un tempérament souverain est apporté par la recherche du bien pour lui-même, dans un pur esprit d'altruisme, ou, selon un mot plus ancien et non moins significatif, de charité.

Qu'on ne se méprenne d'ailleurs pas sur les moyens employés pour réaliser ces fins supérieures. Sans doute l'amélioration des conditions matérielles d'existence n'apparaît-elle plus ici que comme le soutien du relèvement moral des indigènes. Encore n'est-elle nullement négligée et fait-elle même l'objet principal, par la pratique du travail, sous ses formes les plus multiples, de l'œuvre d'évangélisation et de régénération. C'est ainsi que chaque mission, à l'égal des stations administratives, agricoles, militaires, devient un centre d'activité au sein de l'inerte sauvagerie. A ce titre seul, le service rendu est marquant. 
Qu'il s'agisse d'initier les noirs à l'exercice des métiers utiles, ou de leur inculquer des notions de morale, c'est à une œuvre foncière d'éducation que se ramène toujours la tâche des missionnaires. A cet égard leurs méthodes diffèrent beaucoup les unes des autres. Il importe de distinguer entre catholiques et protestants, et, en considérant isolément les catholiques, entre les divers ordres religieux qui se partagent l'évangélisation du Congo. Ces distinctions ne président pas, faut-il le dire? à un plan absolu de préférences; celles-ci pourront naturellement varier suivant les impressions individuelles de chacun. En indiquant les caractères saillants des catégories rencontrées, il doit cependant être loisible à tout observateur d'exprimer sincèrement ses opinions.

Spécialement, il serait bien difficile à un Belge allant au Congo de ne pas se sentir plus proche d'un missionnaire catholique que d'un missionnaire protestant. Par les mille liens de la communauté d'origine, de la langue, des sympathies intimes, des habitudes d'esprit, des rappels du terroir, le premier sera le compatriote, - et loin du pays, c'est tant, - tandis que le second sera l'étranger, d'accès moins facile et moins ouvert, alors même que vous le comprenez et que vous êtes fondé à rendre hommage à sa cordialité et à sa loyauté. Il vaut beaucoup mieux reconnaître les choses telles qu'elles sont que de provoquer de fâcheux malentendus. Pour notre part, nous estimerions profondément illogique de reprocher à des Anglais ou à des Américains le fait de songer, le cas échéant, par-dessus tout, à leur patrie. Seulement, faisons-en autant de notre côté. 
Ce qui frappe, à première vue, dans les missions catholiques, c'est le caractère d'apostolat que revêt manifestement leur action. Quand on le voudrait, on ne pourrait attribuer à celle-ci d'autre incitation que celle du renoncement volontaire, des souffrances et des sacrifices allègrement acceptés, en vue de récompenses dont pas une ne touchera directement ni immédiatement l'auteur du bien répandu.

Les joies actuelles se réduisent à la réussite de l'œuvre de catéchisation; elles se confondent, pour ainsi dire, avec l'exaltation spirituelle que procure la foi en des fins divines d'outre-mort. Et cela est sensible, qu'on suive la sœur de charité dans l'hôpital où, diligente et douce, elle donne ses soins aux malades; qu'on visite des stations comme Berghe-Sainte-Marie ou NouvelleAnvers où sont élevés des groupes de cinq à six cents enfants des deux sexes; qu'on découvre les méthodes qui, chez les pères jésuites de Kisantu, Kimuenza, Dembo assurent l'infiltration et la pénétration des idées religieuses dans des régions en principe assez rebelles à toute influence morale. J'en passe et des meilleures; mais dans ces notes et impressions je veux rester fidèle à la règle de ne rien rapporter qui ne se rattache à un coin de réalité saisi et fixé par mes souvenirs personnels.

Voici d'abord Berghe-Sainte-Marie, située à l'embouchure du Kasai, en face Kwamouth. La station, pour s'établir et s'étendre, a fait une emprise de près de 800 hectarés sur la brousse. Les champs cultivés qui l'entourent assurent sa subsistance, une briqueterie donne des matériaux pour les constructions durables. 
Parmi les plus belles sont l'église et la maison destinée à Mgr Van Ronslé, d'où la vue porte sur la magnifique nappe d'eau du Congo et du Kasai. Il y a encore l'habitation des sœurs, l'infirmerie, les dépendances, les petites maisons, en briques aussi, où sont installés les ménages indigènes. Courant régulièrement à travers l'agglomération, les allées droites bordées d'ananas, ombragées d'éläis, de citronniers, de papayers, d'arbres à pain, de bananiers. Bien que le sol ne soit pas encore fertile comme l'est la terre continuellement arrosée de la région équatoriole, il ne ressemble plus à la lande du Stanley-Pool. Il se prête à des cultures variées où figurent les patates douces, les arachides, sans omettre le café, dont vingt mille plants attestent une expérience de plus poursuivie avec quelque succès. La base de l'agriculture locale est fournie par le manioc, qu'on se figure malaisément être d'introduction relativement récente au Congo, tant est universel son emploi par les indigènes. Que mangeaient-ils donc auparavant?

Si le côté agricole requiert l'attention à Berghe-SainteMarie, ce n'est pas que la station soit un centre de production. Elle est un centre de colonisation indigène, en ce qu'elle vit sur elle-même, par des éléments qui y ont été réunis artifici-ílement. Le village a été créé par les pères de Scheut, au moyen des enfants qui.leur ont été confiés. Ce ne sont point des natifs de la région. Ils viennent d'un peu partout, des parties éloignées du Haut-Congo le plus souvent. Abandonnés, orphelins, petits captifs, jouets de toutes les vicissitudes qui fondent sur des populations en guerre entre elles, ou en lutte 
contre la conquête blanche, ces enfants sont assez nombreux pour former population. A Berghe, en les prenant de l'âge tendre jusqu'à l'âge adulte, ils sont environ six cents, avec sensiblement plus de filles que de garçons. Les pères Van Leeuwen et Geens, un frère charpentier, trois sœurs élèvent et conduisent tout ce petit monde. A l'égard des plus grands il ne s'agit plus que d'une direction morale. Ce sont des gens mariés. Les jeunes ménages reçoivent une petite maison, avec un champ d'un hectare à faire valoir. L'intérieur des cases indigènes est divisé en deux compartiments par une cloison qui ne s'élève qu'à mi-hauteur du toit. C'est rudimentaire, mais presque luxueux en comparaison de l'habitation authentiquement indigène. Quant au vêtement, - le pagne passé sous les aisselles, chez les filles, ou arrêté à la ceinture chez les garçons, - aussi élémentaire qu'il soit, il marque la même transformation extérieure qui abolit le dénuement du sauvage primitif.

A Nouvelle-Anvers la maison de Scheut possède un établissement qui reproduit les traits déjà relevés à Berghe. Les renseignements recueillis de la bouche de l'excellent père Cneut complètent la physionomie de l'ordre en Afrique. Ici encore, six cents enfants environ reçoivent l'éducation première qui leur permettra de se transformer en ménages chrétiens Ces derniers sont au nombre de soixante-dix; on leur confie la culture de champs atteignant respectivement deux et trois hectares. La vie rustique de nos campagnards est le modèle qu'on s'est proposé, bien évidemment; lïnstruction proprement dite n'est distribuée qu'à quelques boys, les aides et 
serviteurs dont les blancs doivent s'entourer là-bas. Les travaux manuels sont pour le grand nombre et, pour tous, l'enseignement religieux. A raison du mélange extraordinaire des races qui se produit parmi ces communautés d'enfants, une langue indigène unique a été prise comme véhicule des relations sociales; c'est le bobangi, parlé le long de la section la plus étendue du haut fleuve Quant au français, il fait l'objet de leçons élémentaires.

En l'honneur des visiteurs de la mission, les pères avaient amené leur contingent de garçons, les sœurs franciscaines - collaboratrices de l'œuvre de Scheut aux Bangala - leur contingent de filles, devant la maison hospitalière où se reposaient les voyageurs. La revue des écoles commença. Elle ressemblait peu au célèbre tableau si blanc où figure le bourgmestre de Bruxelles. Mais d'Europe en Afrique la distance est grande. Quand tous ces petits noirauds furent réunis : "Saluez! " dit le père; "Bonzour, Moussu! " répondirent les vois argentines en chœur; et c'étaient là les enfants que de bonnes gens soignent et aiment au nom de la profonde parole d'amour et d'humaine égalité... Seront-elles payées de leurs peines?

L'influence est restreinte qui rayonne de ces communautés d'enfants recueillis. Le milieu indigène dans son ensemble n'est presque pas touché par elles. Dans l'immense étendue du Congo, combien ne faudrait-il pas de ces centres pour constituer un noyau tant soit peu appréciable? Pris en eux-mêmes, ils ont quelque consis. tance, voire du décor. Mais que deviendrait l'indigène 
livré à lui-même? Le patronage - car c'en est un n'est-il pas un cadre un peu étroit, trop restrictif des penchants de la constitution physique et mentale du sauvage? Ne lui faut-il pas plus d'air, d'espace, de liberté dans ses mouvements, pour que se développe le sens de sa responsabilité? Autant de questions qui s'imposent à l'éducateur d'âmes et qu'ont abordées d'autres ordres religieux, en cherchant à les résoudre, selon des voies éminemment ingénieuses.

L'énorme étendue du champ ouvert à leur propagande n'est pas le moindre obstacle qu'aient rencontre les missionnaires. Aussi leurs tentatives ont-elles conservé, en général, un caractère limité, local : la constitution de ces premières communautés chrétiennes, entièrement distinctes de la société africaine.

La méthode - et on peut le dire - la politique des pères jésuites vise plus loin. Elle comporte, comme ailleurs, des points d'appui solides, mais ceux-ci sont reliés entre eux par les mailles d'une sorte de réseau indigène. De même que les ganglions du système nerveux ne règlent pas, à eux seuls, la vie du corps, qu'il faut de plus à celui-ci le sang qui circule jusque dans les moindres tissus, de même aux directions imprimées par les stations centrales doivent répondre les actions ou réactions spontanées des naturels, tout l'afflux des mœurs, coutumes, habitudes dont est faite la vie sociale, même chez les primitifs.

Telle apparaît, du moins dans ses grandes lignes, l'œuvre d'évangélisation poursuivie par la Compagnie de Jésus dans le Kwango oriental. Kisantu, Kimuenza, 
Dembo forment tout d'abord les centres d'où part l'impulsion directrice. Sauf les différences en population, en personnel, en ressources, ce qui s'applique à l'une de ces stations s'applique aux autres pareillement, puisqu'elles sont comprises dans un plan d'ensemble dont l'exécution s'opère avec une parfaite unité.

Kimuenza, que nous avons visitée à notre voyage de retour, se présente sous des dehors vraiment séduisants, qui doivent beaucoup au site, l'un des plus beaux du Bas-Congo. Placée en bordure du chemin de fer, non loin de Léopoldville, - à une journée de marche, elle jouit d'une vue étendue. Du plateau où sont édifiés les bâtiments de la mission, l'on domine au loin des fonds et des pentes que couvre une végétation forestière sombre et druc. Une brise salubre évente la hauteur et en rend le séjour très supportable et parfois fort agréable. L'absence d'eau et, en conséquence, de terre arable sont, par contre, des inconvénients sensibles. Il faut chercher l'une et l'autre au bas des raidillons très rapides qui conduisent au ruisseau et aux champs attenants, aménagés surtout en vue de la culture potagère. Les constructions sont réparties en deux quartiers, sous la surveillance respective des pères et des sœurs : le pensionnat des garçons et le pensionnat des filles.

Moins que dans le Haut-Congo, les constructions ne sont faites ici pour étonner. Les ressources en matériaux et en main-d'œuvre sont sinon plus abondantes, du moins plus à portée. Notons cependant le quadrilatère que forment dans la partie principale la maison en briques habitée par le père Liagre et les deux frères 


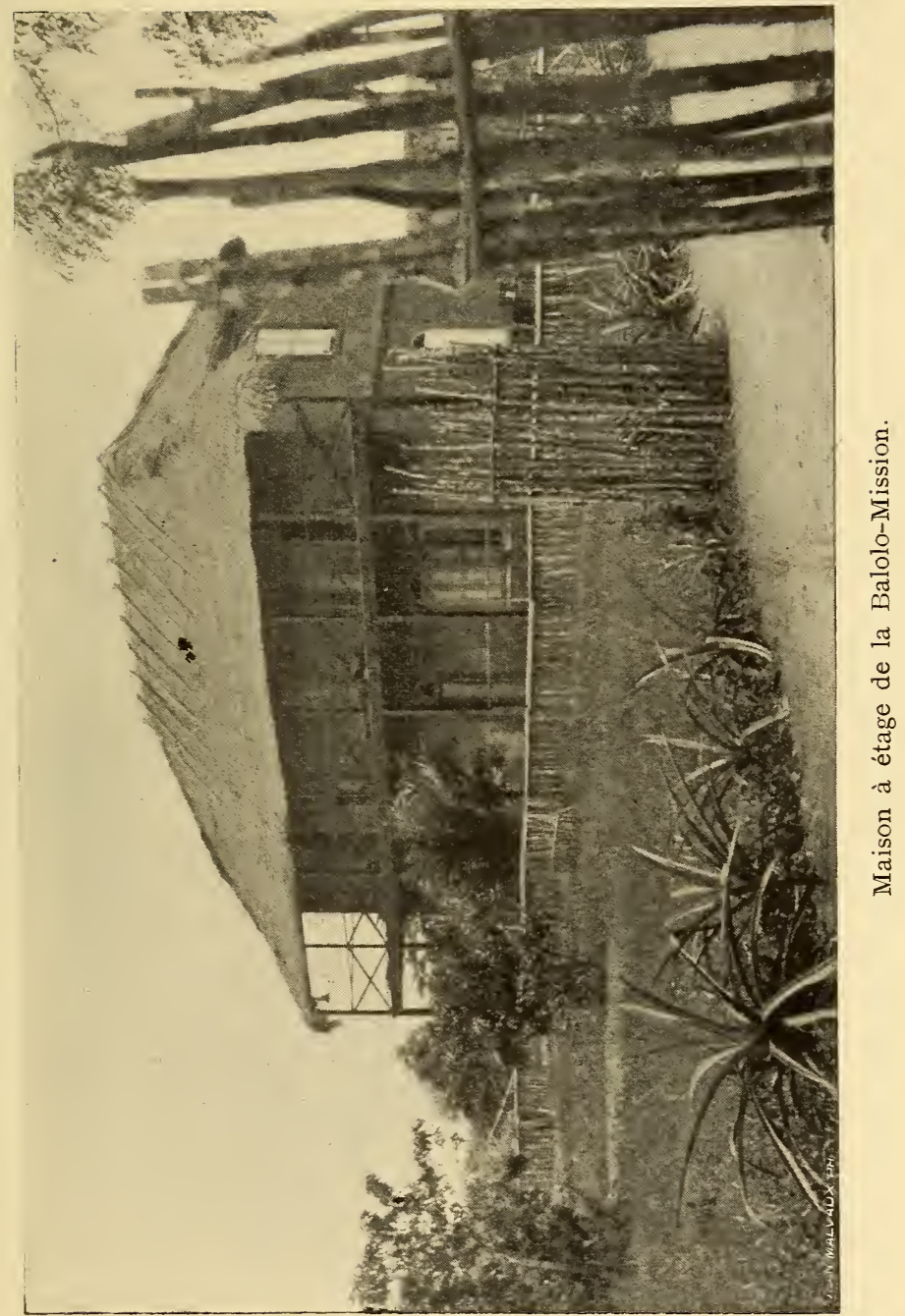



ses adjoints ; la chapelle, en face; sur les autres côtés, les dépendances : cuisine, pharmacie, ateliers de charpentier, les dortoirs des enfants, l'école. Au centre de la place, un mât de pavillon surmonté - attention délicate à l'adresse du bourgmestre de Bruxelles - du drapeau belge. Les couleurs tricolores, pour déférer aux règles protocolaires sans doute, ne sont presque jamais déployées là-bas. Elles n'en deviennent que plus parlantes à côté du drapeau bleu étoilé d'or, aussi haut qu'ait été porté celui-ci depuis quelque vingt ans.

Par des allées joliment tracées on accède, en quelques minutes, au quartier des sœurs. Celles-ci sont au nombre de sept, dont quatre Belges, une Anglaise, une Hollandaise et la supérieure, de nationalité autrichienne. La mine souriante et, chez les dernières arrivées, sans apparence d'anémie ni de fatigue, elles vaquent à leur besogne, présidant aux travaux et aux jeux des négrillonnes, fillettes uniformément vêtues de robes en cotonnade bleu foncé. La maison d'habitation très simple, et plutôt exigüe, la chapelle, l'école, en quelques coups d'œil tout cela est passé en revue. Prise dans son aspect extérieur, Kimuenza rappelle en somme les stations vues précédemment, à cette différence près qu'elle occupe moins d'espace et qu’elle est moins peuplée. Dans une certaine mesure le régime adopté ne diffère pas de celui en vigueur chez les pères de Scheut. La culture, les métiers manuels restent, comme il convient, à la base des programmes d'éducation. L'instruction proprement dite consiste dans le lire et écrire, les quatre opérations, et encore ne sont appelés à cette science primaire que les mieux doués. 
L'entraînement physique est conçu selon des données assez dures. Avec raison, les pères estiment qu'il ne faut pas déshabituer les jeunes noirs de leurs façons de vivre ordinaires. Pour le couchage, des dortoirs où les lits sont figurés par deux longues banquettes en bois adossées au mur, et divisées en compartiments-boxes étroits, munis chacun d'une couverture. Pour l'alimentation, le manioc, les arachides, des légumes indigènes, la viande étant rare et chère. La discipline est sévère et comprend les châtiments corporels.

Où réside l'originalité du système, c'est dans la préparation au genre de vie à mener par les pensionnaires à la sortie de l'établissement. Il ne faudrait pas s'imaginer que chacune des stations de Kisantu (la maison mère), de Kimuenza ou de Dembo soit destinée à se déve. lopper indéfiniment sur elle-même. Kimuenza, par exemple, existe depuis cinq, six ans. Dans dix ans, à supposer qu'elle soit maintenue à l'emplacement actuel, elle ne comprendra pas forcément plus de bâtisses, plus de dépendances immédiates en ateliers, champs de culture. Mais dans l'idée de ses directeurs, son rayon d'action se sera beaucoup élargi. A cette fin nous devons nous demander ce que deviendra sa petite population scolaire - de cent cinquante à deux cents élèves des deux sexes - que nous y avons vue.

Un certain nombre resteront attachés à l'établissement, en qualité d'aides, de serviteurs, d'artisans. Pour les autres, et c'est la catégorie la plus intéressante, un exode au petit pied est prévu. Des ménages seront formés entre adultes, mais loin qu'ils soient retenus 
dans l'enceinte de la mission, ils seront envoyés au dehors à des distances variables, allant jusque dix, quinze lieues et plus. Ils formeront ainsi autour d'elle des postes avancés, où seront combinés à doses égales la sujétion et l'autonomie. En effet, les catéchistes, ou noirs éduqués, n'élisent pas domicile dans les villages mêmes des indigènes que par opposition nous appellerons les sauvages. Ils se fixent à proximité, assez loin pour n'être pas confondus avec eux et pour n'être pas repris par le milieu, et assez près cependant pour nouer avec eux des relations utiles, exercer l'ascendant que leur confèrent leurs connaissances, ainsi que la protection et l'amitié particulière des blancs. Ils deviennent par là-même de précieux collaborateurs pour les missionnaires, leur ouvrant la route, apaisant les méfiances, encourageant les bonnes volontés. Dès à présent il paraîtrait que des noirs sortis des maisons d'éducation que nous décrivons, se font accepter par les "sauvages" comme des chefs, des conseillers autorisés, disposant d'une si réelle influence qu'ils sont même prêts, parfois, à en abuser. Tel ce bon noiraud qui, d'après ce que nous racontait le père Liagre en riant, avait trouvé ingénieux d'employer son crédit très réel à pratiquer l'usure.

Pour parer aux abus et méfaits possibles, résultat du sentiment d'une trop grande indépendance, les pères n'interviennent qu'en vertu de leur autorité morale. Ils font des visites à la ronde, inspectent les fermes-chapelles disséminées dans la région, se font rendre des comptes. Ou bien encore ils convoquent leurs pupilles au chef-lieu, 
à l'occasion des fêtes ou cérémonies; bref, ils tiennent toujours en main les fils ténus qui commandent le système et le font marcher à leur gré.

Par la pénétration, indirecte il est vrai, mais effective du milieu indigène, en même temps que par l'initiative laissée à leurs catéchistes, - qui s'étend jusqu'à l'évangélisation, - les pères jésuites corrigent, à n'en pas douter, ce qu'il y a de factice et de forcé dans le recrutement des enfants confiés aux missions. Non pas que le sort fait à ces derniers, par les religieux, soit jamais mauvais en lui-même; mais le colonisateur doit constamment se préoccuper du problème si difficile de l'adaptation. Ce n'est que lorsque la société autochtone dans son ensemble évolue vers une condition meilleure, que l'expérience qui met en contact deux choses aussi dissemblables que la civilisation et la sauvagerie, peut être considérée comme réussie.

On a si souvent parlé de la destruction fatale des races inférieures en présence des races supérieures qu'on doit saluer avec une joie sincère tout fait qui démontre le contraire. Cette démonstration n'est évidemment pas faite, quand on ne laisse rien subsister des cadres de la vie propre des noirs, alors même qu'on aurait réussi à créer de-ci de-là des centres relativement complets, sur le modèle européen. La preuve est plus convaincante qui résulte de l'expérience instituée dans le Kwango oriental, bien récente au surplus, pour qu'on soit entièrement fixé sur sa valeur intrinsèque. Il ne s'agit d'ailleurs pas ici d'émettre un suffrage, mais seulement d'attirer l'attention sur tous les modes d'action 
de la propagande chrétienne. A ce titre il nous reste à parler des missions protestantes.

Ce sont des Anglais et des Américains qui représentent, pour la presque totalité, les confessions réformées au Congo. La mention de la nationalité des missionnaires n'est pas inutile, car, dans l'espèce, elle caractérise de la façon la plus significative "l'ordre spirituel " dans lequel ils sont engagés, l'idée morale qu'ils cherchent à propager. Non pas que, partant du point de vue chrétien, il faille établir une sorte d'opposition entre protestants et catholiques. Dans le milieu africain, dogmes et doctrines ne sont guère sujets de controverses. Mais les méthodes sont presque antinomiques qu'adoptent les uns et les autres.

La liberté et l'individualisme sont à la base de toute organisation anglo-saxonne. Ces deux principes devaient s'affirmer et s'affirment effectivement dans la propagande religieuse.

Dans le recrutement d'abord. Alors qu'une stricte discipline préside à la répartition des missionnaires catholiques dans les " pays payens ", les missionnaires protestants proviennent de sources captées avec beaucoup moins de minutie. Tel honnête homme qui eût fait un bon commerçant, un hardi voyageur, un colon de ténacité éprouvée, entre délibérément dans les voies du prosélytisme. Il a le zèle et la foi, il peut prêcher et convertir. Il n'appartient pas à un ordre si fermé qu'il ne puisse en sortir, s'il lui plaît, ni surtout qu'il doive renoncer aux perspectives de la vie pratique. Il s'établit, parmi les sauvages, comme " un pionnier de la civili- 
sation ". En règle générale, il est puissamment secondé par les associations dont il est l'émissaire. Il a une situation matérielle assurée. De lui dépendra de l'améliorer, de prospérer, soit en recevant des émoluments plus élevés, proportionnés à la durée de ses services, soit en fondant, de son initiative privée, des entreprises avantageuses.

A cet égard, il ne faudrait cependant pas insister plus que de raison sur le côté " affaires " souvent mis en vedette à propos de missionnaires protestants jouant le rôle de commis-voyageurs de l'expansion commerciale et industrielle de l'Angleterre ou de l'Amérique. A part des individualités qui ne se rangent que de nom parmi les " hommes de Dieu " il faut admettre, chez la plupart, l'existence de mobiles élevés, le souci du bien faire, l'espoir généreux de voir une fraction déshéritée de l'humanité sortir, grâce à leurs efforts, de sa condition malheureuse. Lors même que la vocation apostolique la plus pure - celle d'un père Damiens - n'animerait pas indistinctement chacun des missionnaires protestants, on ne saurait leur refuser la qualité essentielle de braves gens. Et, d'autre part, ceux qui les soutiennent, qui alimentent de leurs souscriptions les budgets considérables de la propagande confessionnelle, n'obéissent évidemment qu'à des motifs désintéressés

Les établissements des différentes sociétés en activité sur le territoire de l'État Indépendant portent bien leur marque originelle. A quelque Église qu'ils se rattachent, ils reproduisent tous certains traits communs. La mission constitue d'abord pour ses occupants un home 
véritable, une installation d'apparence permanente et définitive. Le maximum de confort compatible avec les difficultés inhérentes à l'éloignement et à l'isolement est réalisé. L'habitation, dans son aspect extérieur comme dans ses aménagements, répond aux règles d'une hygiène judicieusement entendue; elle peut servir de "type " aux constructions européennes à édifier dans la région. Le rez-de-chaussée est surélevé, à bonne distance du sol si fréquemment pénétré de germes malariques. Assise tantôt sur de hauts pilots, tantôt sur des voûtes maçonnées en berceau, la maison, parfois à étage, est ventilée, saine et spacieuse. L'intérieur n'est pas moins soigné : chambres disposées, parées, meublées, tapissées au goût de gens que la civilisation a façonnés. Ne médisons point de leurs besoins. Ils sont en corrélation avec des habitudes morales évidemment supérieures.

Il ne s'agit d'ailleurs point de luxe, cela s'entend, mais d'objets utiles à l'existence quotidienne, voire nécessaires à la lutte contre tant d'éléments hostiles : le climat, les maladies, les multiples vicissitudes du séjour colonial. C'est de la prévoyance, ni plus ni moins, que d'avoir toujours à la main provisions, ravitaillements, remèdes, sans omettre les ressources locales à créer ou à développer : le jardin potager, le poulailler, l'élève du petit bétail.

Tout cela réuni forme "oasis " dans la sauvagerie environnante. Et si la chose mérite d'être mise en relief, c'est que les conditions tant discutées de l'acclimatement en ressortent singulièrement modifiées. Les habitants de 
ces missions sont infiniment mieux armés que ne le sont la plupart des autres coloniaux pour résister aux influences délétères d'ordre physique ou moral. Ils mènent la vie de famille. Le berceau installé sur la véranda, la réception courtoise que vous ménage une maîtresse de maison vous offrant le thé dans son salon deviennent dès lors des faits très importants, dont la signification n'échappera à personne. Les missionnaires protestants ont donné_là un'exemple que des"laïques ont pu suivre par après, pour s'en féliciter hautement.

Mais, dira-t-on, que idevient dans ce train de vie, très sortable, leur tâche particulière d'apostolat? Quand ils auront créé une jolie villa, qu'ils auront dressé à leur usage personnel des serviteurs noirs en nombre suffisant, qu'ils se seront créé des occupations intéressantes, en quoi auront-ils coopéré au relèvement de la masse indigène?

L'objection, pour être d'apparence spécieuse, ne tient pas compte d'éléments importants de la question. Les missionnaires protestants s'installent, pour ainsi parler, au plus épais de la population noire, à proximité d'agglomérations pouvant compter quatre mille, cinq mille habitants et davantage. Sous la protection très efficace des autorités de l'État, ils s'adressent directement aux " sauvages ". Ils n'exigent rien d'eux et, par conséquent, n'en obtiennent pas grand'chose. Mais ils leur ont annoncé qu'ils sont à leur disposition. Si des enfants veulent s'instruire, recevoir des notions utiles, l'église-école est proche; si les adultes veulent acquérir étoffes, marchandises, articles de tout genre, il leur 
appartient de les gagner en exécutant tel ou tel travail, en apportant des vivres, en fournissant des prestations quelconques; s'il y a des malades, qu'ils viennent se faire soigner par l'homme blanc, grand guérisseur par fonctions.

Ainsi se nouent des relations qui, à la longue, deviennent très suivies et empreintes d'une visible cordialité. Pour juger de ce qu'elles produisent ou, tout au moins, de ce qu'elles permettent d'accomplir, la station de Bolobo, de l'English Baptist Missionary Society, est particulièrement bien placée. Dirigée par l'un des plus anciens et des plus méritants Congolais, l'éminent Grenfell, elle présente, en premier lieu, un développement local très remarquable. Par l'importance de ses constructions, maison, église, dépendances, par ses avenues tracées dans la brousse, elle témoigne déjà de patients efforts.

Ce qui vaut mieux, la population du voisinage, très dense, a été parmi les plus empressées que nous ayons rencontrées à entrer en rapports avec le bateau qui abordait à leur rive. Le jour de notre passage, celle-ci était transformée en marché, tant étaient grandes l'affluence et l'animation des noirs qui voulaient acheter, vendre, négocier. Outre la briqueterie, qui est de rigueur dans tous les postes du Haut-Congo, outre la forge où l'on peut réparer jusqu'aux engins délicats du petit vapeur appartenant à la mission, Bolobo possède, et c'est son titre de gloire, une imprimerie. Des noirs du pays sont devenus typographes, prouvant qu'il n'est point de métier, aussi délicat soit-il, qu'ils soient inaptes 
à apprendre. Et ils impriment des bibles, des livres de lecture, des grammaires en langue indigène, dont l'étude par les blancs comme par les noirs sera de la plus grande utilité.

Au point de vue des " extensions " futures, de la pénétration intime de la société primitive, cette organisation, quoique alimentée par un budget annuel de 13 à 14 mille livres, ne laisse pas d'être réduite à un objet assez restreint. Son action, en vertu du système adopté, il faut le reconnaître, est limitée. Elle procède - et c'est le terme qu'on pourrait appliquer à toutes les missions protestantes - par rayonnement très lent de proche en proche. Chacune des stations dirigées en pleine barbarie est un foyer, si l'on veut, de bien-être matériel et d'influence morale. Dans la mesure où les noirs sauront apprécier la force civilisatrice qui se dégage de ce foyer, ils en ressentiront les bienfaits. La question est seulement de savoir si à défaut d'une initiation préalable, où la contrainte mitigée, en d'autres termes, l'éducation de l'enfance, jouera son rôle, ils sortiront de leur apathie foncière. Rien que l'exemple suffira-t-il? La cause n'est pas encore décidée.

Quelques centaines d'enfants sachant lire et écrire, quelques milliers de baptisés et d'auditeurs aux prêches ne forment pas une classe dirigeante, capable d'entraîner et de relever la masse, voilà qui est certain. Cependant des hommes d'expérience, comme M. Grenfell, croient en l'avenir, après deux ou trois générations écoulées; M. Kenredsmith, à Upoto, estime que les indigènes, instruits par la mission, déploient aussitôt, 
vis-à-vis de leurs congénères, une supériorité très marquée. Ces appréciations sont formulées par des hommes qui ne cherchent à grossir ni leurs mérites ni leurs succès; et comme ils ne s'abstraient pas des réalités ambiantes, qu'au contraire ces missionnaires protestants continuent à suivre de près les fluctuations de la vie universelle, jusqu'aux événements politiques de Cuba pour lesquels ils se passionnaient tous, - que d'autres s'adonnent à des études scientifiques, géographie, ethnographie, philologie, procurant à l'esprit des directions positives et nullement chimériques, il est juste, pensons-nous, de prendre leurs avis en bonne et due considération.

Ils cadrent entièrement avec ceux qu'avec une éagle modération, et non moins de confiante sérénité, émettaient les dignes pères de Scheut ou les pères jésuites. Le principe qui les unit tous dans une certaine mesure, c'est moins, redisons-le, la recherche de fins utilitaires pour l'obtention desquelles les pouvoirs publics ainsi que les agents de la pénétration économique sont en somme mieux armés qu'eux, c'est moins cela que la poursuite d'un idéal à coup sûr admirable : le relèvement moral de la race de Cham. Aucune grande œuvre humaine ne peut se passer d'idéal, et il est heureux que dans la colonisation il puisse s'affirmer avec éclat. A ce titre, les missions, de quelque confession qu'elles se réclament, ont droit au Congo à l'hommage respectueux de tous les esprits impartiaux. 



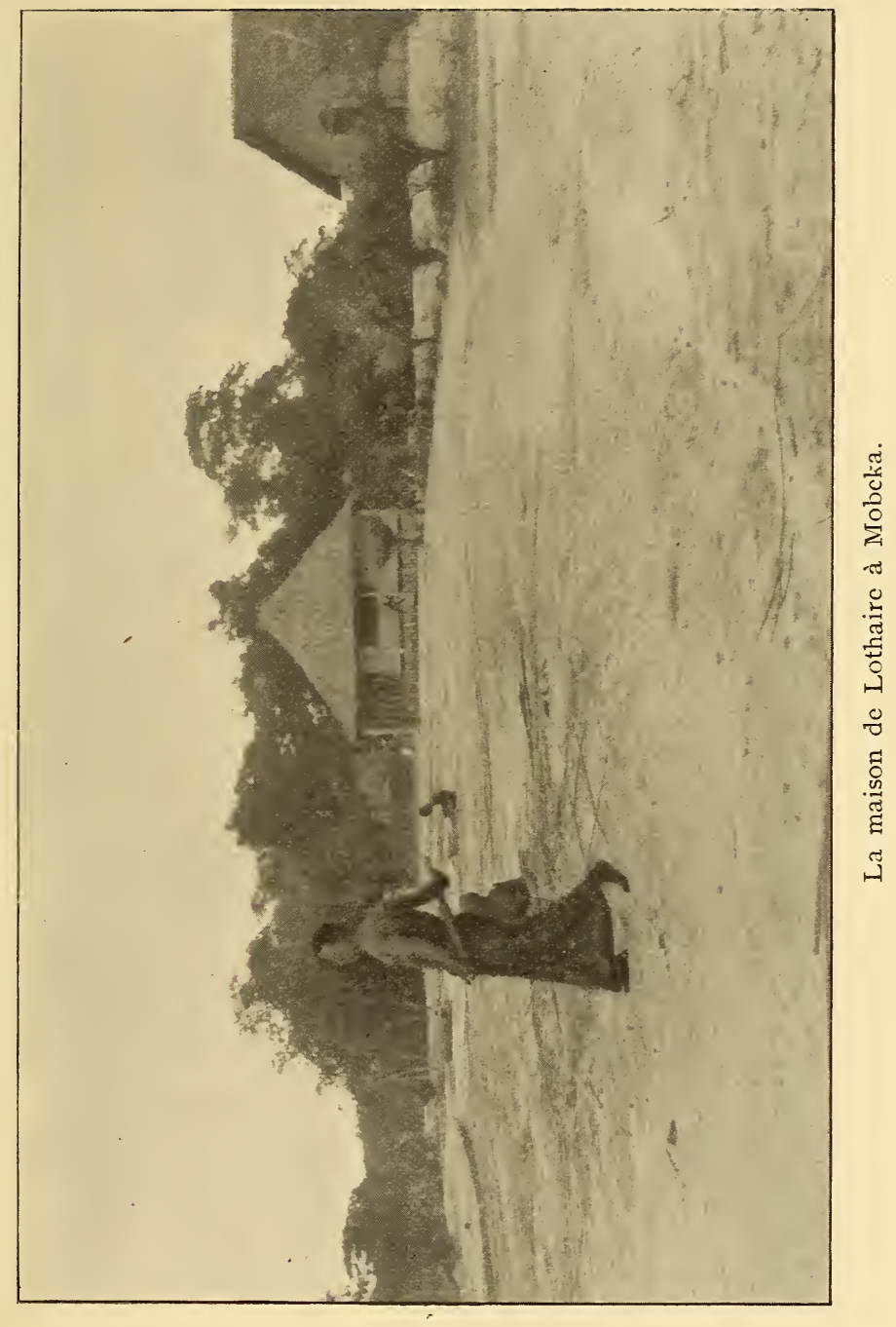





\section{X}

\section{LES FACTORERIES}

Le commerce, au Congo, a suivi les routes que lui ont frayées les pouvoirs publics. En 1879 , époque où Stanley remontait le bas fleuve et la région des cataractes pour le compte du Comité d'études, - au prix de quelles difficultés, on le sait, - pendant toute la durée de l'Association internationale, héritière du Comité d'études, et jusqu'à la constitution de l'État Indépendant, réalisée en r 885 , durant la Conférence de Berlin, le vaste bassin de l'Afrique centrale est demeuré fermé à toute pénétration individuelle ou collective d'ordre commercial. Seule une étroite bande côtière, près de l'embouchure même du fleuve, était l'objet d'une 
exploitation locale de la part de traitants qui ne connaissaient plus les temps dorés et d'ailleurs abominables du trafic des esclaves.

Mais lorsqu'eut été franchie la barrière des monts de Cristal et que le réseau navigable de l'intérieur, avec ses ressources énormes, eut été révélé au monde, hommes et capitaux se trouvèrent pour en tirer parti. Les débuts, qui datent de 1888, furent modestes. Aujourd'hui, d'après les dernières données publiées, une trentaine de sociétés, réunissant des capitaux pour 70 millions de francs environ (valeur de fondation), sont en pleine activité dans cette terre si longtemps dédaignée ou méconnue. Le mouvement des échanges, nul il y a vingtcinq ans, entre le Congo et l'extérieur, atteint 5o millions. En laissant à l'État la part qui lui revient directement ou indirectement dans ce total, il reste pour le commerce proprement dit, le commerce privé, un champ d'action dont la valeur économique ne saurait plus être contestée. L'ouverture de nouveaux marchés a été la conséquence directe des grandioses efforts de pénétration des continuateurs de Stanley.

C'est le long de la route, jalonnée par tant de vaillants agents, officiers et explorateurs, que se sont installés, tout naturellement, les premiers factoreriens. Le cours même du fleuve est devenu, pour le commerce, ce qu'il est pour l'organisme politique : l'artère principale où aboutit tout l'afflux de vie du corps entier. Qui a vu et visité les établissements de telle ou telle firme importante, dans le voyage du Pool aux Falls, se rend aisément compte du fonctionnement du mécanisme com- 
mercial jusque dans les postes éloignés. Les aspects et les caractères par où il se manifeste diffèrent, à vrai dire, de ce qu'on a pu voir dans les services d'État, dans les camps, dans les missions. Les représentants de chaque groupe social conservent, même en Afrique, les traits professionnels qui sont comme une marque indélébile.

L'installation du factorerien indique le choix d'un genre de vie où puissent se combiner liberté d'allures, gains plus larges, goûts aventureux. De là, chez le colon de cette origine, le souci de quelque permanence dans ses habitudes. Il n'est pas détaché en Afrique; au contraire, et presque par définition, il doit sy attacher pendant le temps relativement long qui lui est nécessaire pour s'y faire sa position. Du moment qu'il s'élève un peu dans la hiérarchie des agents, il s'assurera un confort comparable à celui qui existe dans les missions protestantes. A cet égard, Kinshasa, où est située la station centrale de l'S. A. B., tout comme la factorerie hollandaise de M. Greshoff, sur la rive opposée du StanleyPool, près de Brazzaville, apparaissent comme des oasis de civilisation aimable dans un milieu par trop réduit au strict nécessaire. Ce ne sont cependant point des efféminés qui s'entendent à agrémenter ainsi leur existence quotidienne de quelque raffinement, portant sur l'habitation et son aménagement intérieur, sur le mobilier, le service de table, le billard, la bibliothèque, le piano, instrument ô combien rare sur le Haut-Congo. A Kinshasa réside le $\mathrm{D}^{\mathrm{r}}$ Briart, l'un des compagnons d'Alex. Delcommune dans la mémorable exploration 
du Katanga, un vieil Africain déjà malgré son âge, d'humeur et de santé également solides, bref un " Congolais ") heureux. A côté de lui, et pour le suppléer dans la direction de l'S. A. B , M. Thiery, qui n'est pas loin de ses dix années d'Afrique et qui, pendant son dernier séjour, n'a pas craint d'emmener avec lui sa jeune femme. A positions égales, l'agent commercial a donc l'air moins " campé " dans le pays que l'agent de l'ordre administratif, militaire ou judiciaire. Son avancement, ses déplacements n'affectent pas au même degré l'apparence d'un changement de garnison à opérer avec le minimum possible d'impedimenta.

Si le côté matériel présente, dans le groupe colonial des factoreriens, des particularités bien reconnaissables, le côté psychologique n'est pas moins curieux à observer. Chez la plupart il n'existe que des préoccupations très limitées, peu ou point de vues générales. La politique de l'État leur plaît médiocrement, parce qu'elle met obstacle à des pratiques qui paraissent naturelles en pays neuf. En raison des difficultés qu'ils ont à vaincre, dans l'exercice de leur négoce, il leur paraît exorbitant que des contraintes et des défenses aient été imposées dans l'intérêt de la chose publique. L'idéal, pour eux, eût été qu'on laissât à tout nouvel arrivant le droit de se tailler sa part à sa guise en terre vierge, sauf à acquitter de légères redevances au petit dieu État. Pas d'expéditions militaires, cela s'entend, pas de juges non plus pour intervenir dans les démêlés personnels entre employeurs blancs et noirs, pas de propriété publique limitant la jouissance du premier occupant; 
en résumé, une anarchie tempérée par les sentiments plus ou moins bienveillants d'un chacun. Et dans ce système sont confondus liberté du commerce et droit de propriété, occupation des terres vacantes et appropriation individuelle, concessions et privilèges.

Pour être juste, il y a une tendance assez accusée à rétrécir le champ des récriminations. Les droits de l'État comme tel ont été mis si clairement hors de conteste, sa force et son crédit s'affirment chaque jour avec une si évidente énergie, que seuls des retardataires agitent encore les épouvantails de jadis. Le plus souvent c'est dans la limite d'intérêts matériels nettement circonscrits que se poursuit une opposition, dont certains éléments méritent d'être considérés. Du moment qu'il ne s'agit plus de questions de principe, mais de questions d'applications, les méthodes doivent être jugées par les fruits qu'elles produisent; et, en outre, les situations de fait étant essentiellement variables, on conçoit qu'on change, le cas échéant, de méthode, comme l'a fait précisément l'État du Congo, dans cette question si souvent débattue de l'exploitation coloniale.

Certaines idées directrices peuvent et doivent présider à la mise en valeur d'un domaine colonial ; mais par là il faut se garder d'entendre un système rigoureux, conçu selon des formules géométriques et applicable en tous temps et en tous lieux.

L'humanité, la bienveillance, la modération, telles sont les qualités morales qui se recommandent par ellesmêmes et qui auront, à l'occasion, une bien autre efficacité, ainsi, dans les relations avec les indigènes, que 
les règlements les mieux faits. C'est à l'action personnelle de l'individu, à sa politique qu'on jugera de la valeur des principes suivis. Les questions de fait dominent tout le reste et leur force probante est invincible, du moment qu'ils sont pris dans leur signification générale. C'est d'ailleurs là que réside la difficulté. Combien d'appréciations erronées uniquement parce qu'on s'est arrêté aux détails, aux cas particuliers, au lieu de conclure d'après les vues et les résultats d'ensemble.

Voilà une terre neuve, le Congo, qui, au point de vue spécial où nous nous plaçons, le rapport, n’a rien donné jusque dans un temps très rapproché de nous.

Cependant son sol est fertile en produits naturels, sa population est relativement dense. Pour récolter ces richesses, il ne faut pas des efforts démesurés, et d'autre part, quoi qu'on ai dit, les indigènes sont susceptibles d'éprouver des besoins nouveaux et de vouloir les satisfaire. Étant de constitution robuste, ils paraissent tout désignés pour fournir aux blancs une certaine somme de travail qui leur apportera des avantages corrélatifs se traduisant par une augmentation indubitable de bienêtre, de prospérité matérielle. L'association des deux éléments est jugée unanimement indispensable; à son défaut, le noir, ne recevant aucun stimulant, ne progressera jamais, et le blanc ne pouvant entreprendre de travailler de ses bras sous les tropiques, n'aura qu'à abandonner la partie.

En vue de réaliser l'association désirée, dans quelles voies convient-il de s'engager? Il en existe plusieurs, mais assurément aucune d'elles n'est dépourvue de sérieux 
obstacles. Sur un point l'on peut encore se dire d'accord, c'est que l'initiative strictement privée eût été impuissante à les franchir. Le passé du Congo, durant trois siècles, l'atteste surabondamment. Une puissance politique était requise qui fût pourvue de moyens d'action appropriés. Cette personne morale ne peut être que l'État. Que celui-ci agisse directement ou par délégation, peu importe. Même l'État Indépendant, de formation si originale, ne fait pas exception à la règle. S'il trouve ses fondements juridiques en lui-même, il n'emprunte pas moins à une nation européenne une partie de ses ressources et encore plus ses forces vives de commandement et d'exécution.

L'État étant constitué, il ne pouvait venir à l'esprit de personne de lui substituer un délégué général celui que représente à l'époque moderne la compagnie à charte - pour inaugurer la colonisation proprement dite. Le travail préparatoire consistant à ouvrir une région, à la reconnaître, à établir des communications, à nouer les premières relations avec les indigènes, avait été accompli par le Comité d'Études et par l'Association Internationale. Or, c'est précisément pour ces objets-là que se sont créées le plus souvent les compagnies à charte. Si leur existence est prolongée au delà du délai nécessaire, c'est que par l'acte de concession on veut leur laisser le temps de rémunérer les capitaux engagés, et aussi démontrer par expérience pratique la valeur de l'acquisition, opérée par leur intermédiaire.

A mesure que cet état provisoire s'étend sur une période plus longue, les inconvénients en deviennent 
plus sensibles. De nature mixte, à la fois politique et commerciale, la compagnie à charte court le risque de pencher d'un côté ou de l'autre, au détriment du juste équilibre. Trop préoccupée des intérêts des actionnaires, elle néglige son rôle de puissance publique; trop hardie en politique, elle se trouve bientôt devant des tâches qui excèdent ses forces, qui l'épuisent et qui la ruinent. Dans tous les cas c'est la liquidation forcée à des conditions plus ou moins favorables, quand intervient d'office l'État souverain et délégant qui exerce ses droits de reprise.

Au Congo belge, le système des compagnies à charte était d'autant moins viable qu'on se demande en vain quels auraient été, en dehors des éléments réunis par l'État lui-même, les hommes et les capitaux venus de Belgique qui auraient assuré le bon fonctionnement de l'organisme? Il importe, par ailleurs, de s'élever contre le préjugé qui consiste à attribuer aux grandes compagnies des procédés moins fiscaux, des allures foncièrement plus pacifiques, - en un mot la politique du commerce opposée à la politique de la conquête. En réalité, il n'existe pas tant de moyens divers pour ouvrir, à la civilisation, une contrée vouée à l'anarchie primitive et sauvage, c'est-à-dire à la guerre perpétuelle, au pillage, aux plus monstrueuses coutumes. Dans ce cas, la force qui est aux mains des civilisés incarne le droit. Le tout est qu'ils n'en abusent point. Et s'il y a des abus, prétendra-t-on qu'ils soient moins graves dans la Nigeria, dans la Rhodesia, comme auparavant aussi dans l'Afrique orientale, tous territoires à charte, que dans les pays soumis étroitement aux lois d'un État souverain? Le 
gin sur le Niger, les exécutions du Matebeleland, les luttes religieuses de l'Uganda et les sanglantes répressions qui en furent la suite, sans compter l'agression inouie contre le Transvaal, sont autant de réponses à la question.

Les intérêts privés, ceux des ressortissants mêmes de l'État colonisateur qui a octroyé la charte, ne sont pas mieux sauvegardés. Pour peu que la concession dépasse certaines limites, toute une partie du commerce métropolitain réclame contre l'exclusion dont elle est l'objet au bénéfice d'une société privilégiée, munie d'un incontestable monopole.

Pour administrer et gouverner, l'État - du moins celui qui régit les pays civilisés - est plus qualifié qu'un collège de particuliers, entrepreneurs de colonisation. Pour régler les besoins économiques, et notamment ceux du commerce, en pays neuf, sans compromettre ni les droits ni les intérêts de la communauté autochtone, ni les besoins légitimes d'expansion des Européens, l'État encore n'est certes pas inférieur à la Compagnie. Nous avons pu admettre qu'abandonnés à eux-mêmes, des individus isolés n'ont pour ainsi dire pas de prise sur le milieu africain, tant sont redoutables les difficultés matérielles à surmonter et hostiles à priori les dispositions des indigènes vis-à-vis de l'étranger, de l'intrus, l'ennemi par définition. La question est donc à poser si l'État doit se faire directement exploitant de son domaine. Même le décentralisateur le plus convaincu ne saurait répondre par une simple négation. L'État connaît ou est censé connaître l'étendue de ses réserves; il sait les 
aptitudes des populations, leurs besoins, leurs aspirations, leurs répugnances; il apprécie les bases premières d'après lesquelles peuvent être fixées approximativement les lois de l'offre et de la demande. Bref! son action régulatrice s'exerce d'après des points de repère que seul il est capable de relever, ménager du présent et soucieux de l'avenir. Pour que l'État puisse remplir son rôle, nous le supposons dès l'abord fortement constitué et, pour tout dire, disposant d'un budget suffisant. Au Congo, le sol lui donneles ressources nécessaires, notamment la forêt vierge où abondent les lianes de caoutchouc. Propriétaire du fonds, il a le droit évident d'en user pour tout ce qui n'a pas fait l'objet d'une appropriation privée antérieure. La forêt vierge est terre vacante, et plus encore la liane était chose vacante avant la prise de possession du Congo par l'État colonisateur. Les noirs qui habitaient par places la forêt l'ignoraient, au même titre que les hommes blancs qui n'y avaient jamais pénétré.

Faut-il conclure de là que la régie doive être étendue à la forêt équatoriale entière? Non, n'est-ce pas; car le but de la colonisation est de provoquer l'afflux des hommes et des capitaux de la métropole vers des entreprises nouvelles. Aussi bien agencée que soit la machine d'État, elle ne peut poourvoir à tous les besoins économiques. A côté d'elle fonctionneront d'autres rouages plus simples et plus souples.

Dans l'ordre de l'exploitation coloniale, ce seront des associations de capitaux, des sociétés commerciales semblables à celles qui ont assuré le prodigieux essor de tant de nations industrieuses dans le monde. 
On conçoit néanmoins que le type normal reçoive quelques modifications On ne fait pas le commerce au Congo comme en Belgique. Il y a toujours cette différence essentielle que les nègres sont des sauvages, voire des cannibales, qui, dans leur premier mouvement, leur réaction si l'on veut, se refusent obstinément à ce qu'on leur demande.

Étant admis qu'il faille persévérer dans le mouvement de pénétration, des mesures de police préventive ou répressive vont se mêler aux opérations commerciales, sans qu'il dépende de personne de n'y avoir point recours. En effet, ce serait une profonde erreur de croire qu'en l'absence de toute contrainte, de toute réquisition, l'indigène, chez qui arrive le blanc, inconnu la veille, doive nécessairement respecter celui-ci, l'accueillir avec une particulière cordialité. Le cas opposé est bien plutôt la règle : la défiance, suivie de provocation, la résistance violente, l'attaque traîtresse. Quand on sait cela, et il est facile de l'apprendre, on hésite à parler de révoltes sanglantes de populations prétendument surmenées, exploitées, pressurées par les sociétés commerciales directement dépendantes de l'État. Loin que les factoreriens, qui ne disposent pas de cette aide, en critiquent l'emploi, ils se plaignent d'en être privés et en conséquence d'avoir moins d'autorité, de prestige, de crédit auprès des indigènes.

Est-ce à dire que des excès ne se commettent jamais et qu'on ne doive espérer du temps un meilleur régime que le régime actuel? C'est ce que personne n'oserait affirmer. Mais d'ores et déjà il est permis de soutenir que 
ni la régie par l'État, ni la société concessionnaire et, dans une certaine mesure, associée de l'État, ne sont des systèmes condamnables en eux-mêmes.

Dans notre " milieu " européen, les catégories sociales sont réparties dans des compartiments en quelque sorte étanches. Et surtout il y a division absolue entres les agents, quels qu'ils soient, qui détiennent une parcelle de l'autorité publique, et les citoyens de divers ordres - la masse - agissant à titre privé. Un commerçant par exemple n'est que cela; quand il acquitte vis à vis de l'État sa quote-part de contribution, il accomplit un acte qui, ni de près ni de loin, ne touche à l'exercice de sa profession.

Il en va autrement dans un pays neuf, dépourvu encore des cadres réguliers dont sont munis les communautés plus avancées en civilisation. Prenez par exemple un indigène de la forêt équatoriale du Congo qui apporte dans un poste établi par les blancs une quantité déterminée de caoutchouc, et demandez-vous ce qu'il est, à quel titre il se présente là devant vous? La question paraît bien simple à première vue et cependant elle comporte plusieurs réponses passablement contradictoires. Ce noir livre un produit marchand, l'un des seuls qui soient objet de trafic dans le centre africain. Qui va en prendre réception? Un traitant? Peut-être. Mais il est possible aussi que ce soit un percepteur. Les impôts, dans une contrée où il n'existe pas de numéraire, se paient en nature. Des deux qualités apparentes de ce fournisseur de caoutchouc, laquelle doit l'emporter? Cela dépendra des circonstances. Il faut d'abord 
tenir compte de ce fait que de sa propre initiative, le nègre n'aurait rien apporté du tout. Il a fallu user sinon de contrainte, du moins d'une certaine pression pour amener ce résultat. Le négociant européen, réduit à ses forces propres, individuelles et particulières, eût certes échoué dans l'entreprise. S'il se fut avancé trop à l'aventure, dans ses démarches pacifiquement intéressées auprès des indigènes, il eût été probablement capturé, dépouillé, massacré. Sous une forme ou l'autre, seul un pouvoir fort était capable d'avoir raison des défiances ou de l'hostilité des habitants. Il s'est installé d'autorité, a manifesté l'intention formelle d'être respecté, de maintenir la sécurité et l'ordre au profit de tous. Ce pouvoir - c'est le souverain - a donc " ouvert le pays " sinon à la science géographique où parfois des initiatives privées l'ont précédé, du moins à l'exploitation.

Les efforts faits à cette fin ont coûté cher, ont souvent entraîné de douloureux sacrifices. La première compensation - et encore est-elle bien faible au début - consiste dans le revenu, non exploité jusque-là, de cette terre neuve. Le produit de la forêt recueilli et livré par l'indigène sera dès lors considéré comme l'impôt le plus légitime.

Est-ce à dire que c'est pour le plaisir de faire de l'administration qu'on colonise? Point n'est besoin de répondre aux plaisanteries faciles bâties sur ce thème Le but que se propose toute puissance colonisatrice c'est de profiter, en les appelant à elle, des forces vives que procurent l'industrie et le commerce d'un peuple 
riche. Seulement il convient de reconnaître que, le cas échéant, il y aura concurrence relative entre l'intérêt public et l'intérêt privé et que celui-ci doit le céder au premier, au moins pendant une certaine période de préparation et de transition.

En reprenant l'exemple de tantôt, du noir livrant du caoutchouc, un second fait va étonner. Alors même qu'il fait fonction de contribuable, il reçoit une rémunération, qui proportionnellement, non pas à la valeur de la marchandise fournie, mais à celle du travail accompli, ou à celle des besoins d'existence, est assez élevée. Voilà à nouveau toutes nos notions d'économie politique brouillées. Prix d'achat et contribution sont deux choses très différentes. Pourquoi l'État n'exige-t-il pas la prestation à titre gratuit, sauf à la limiter, en quantité, et sauf à laisser l'excédent à la libre disposition des indigènes pour que ceux-ci s'en défassent à un prix réellement marchand, - celui de l'offre, - entre les mains des négociants européens trafiquant pour leur compte personnel?

L'objection est spécieuse et cependant elle ne résiste guère à l'examen.

Le principe même de la rémunération est excellent. Il fallait éveiller l'intérêt de l'indigène par l'appât d'une sorte de prime. Déjà peu soucieux de travailler, quand il a commencé à apprécier les avantages des articles d'échange dont on le paie, il n'aurait certes jamais rien donné ni en nature ni en prestation, s'il n'eut reçu une contre-valeur. A moins de le considérer comme corvéable et taillable à merci, et d'instituer en définitive un 
régime intolérable d'oppression. C'est ce qui n'a jamais été ordonné sur aucun point du territoire de l'État Indépendant. La rémunération étant admise et impliquant, au sens strictement juridique, une libéralité, elle rentre dans les frais généraux de la régie du domaine. Dans cette limite, l'État n'a plus à s'occuper d'un prix marchand. Il n'a plus qu'à envisager la capacité contributive des indigènes mis en rapport avec les besoins budgétaires. Dans tel district un travail $x$ et une quantité $z$ de marchandises représentent un effort minime exigé de l'indigène ; ailleurs, au contraire, un effort considérable.

L'imposition serait le plus souvent dérisoire pour un contribuable d'Europe. Mais, même au Congo, elle sera supportée avec plus ou moins de facilité, suivant le degré d'avancement économique des populations. Quand celles-ci se font consommatrices, éprouvent des besoins grandissants, - et tel est le cas partout où il y a des relations suivies entre blancs et noirs, - travail et prestations en nature s'obtiennent en proportions relativement satisfaisantes. Quand l'Européen arrive pour la première fois parmi des tribus anthropophages, n'ayant aucune idée et par conséquent aucun désir des avantages tout élémentaires de la civilisation, de longs palabres sont nécessaires pour créer un mince courant d'activité, le besoin du " meilleur vivre ".

Question d'application, d'équilibre que tout cela et c'est pourquoi le tact politique allié à beaucoup de fermeté vaut infiniment mieux que les plus beaux systèmes. La réglementation ne doit intervenir que quand 
les cas particuliers sont assez nombreux pour être en certaine manière codifiés. Supposons un district entièrement pacifié, d'accès et de séjour faciles aux individus isolés, habité par des indigènes disposés à vendre et à acheter. Il se peut que par le libre jeu des lois, de l'offre et de la demande, s'édifient assez de fortunes particulières pour alimenter, en impôts réguliers frappant les objets in commercio, les caisses de l'État. Eh bien! cette situation éminemment favorable amènera des résultats analogues à ceux qui existent dans tous les pays organisés. L'État n'aura plus besoin de se faire exploitant. Le fisc se substituera à la régie proprement dite. Peut-être ne faudra-t-il plus de concessions privilégiées; les petites initiatives individuelles ou syndiquées prendront la place des grandes associations de capitaux, de ces organismes où l'État et les sociétés doivent s'unir en partie politiquement, en partie économiquement.

Ce temps, faut-il le dire? n'est pas encore arrivé, du moins pour l'ensemble du domaine congolais.

Actuellement l'intérêt de l'État, et son intérêt absolument légitime, est de faire produire au territoire ouvert par ses soins. Dans les différents systèmes adoptés jusqu'à ce jour cet intérêt est le même. La régie peut rapporter le plus, mais elle a le tort d'être trop exclusive et de laisser sans emploi, alors qu'elles sont disponibles, les forces commerciales de la métropole. C'est pourquoi, en dehors des domaines directement administrés par le gouvernement congolais, il importait que des sociétés prissent part à la mise en valeur des richesses du Congo. Ce n'est point une libéralité qu'on 
leur fait, car elles apportent des hommes et des capitaux à l'association formée avec l'État. Celui-ci est coopérant, actionnaire, administrateur, par ses délégués, au même titre que peut l'être l'Angleterre dans le canal de Suez, ou un État quelconque dans des combinaisons financières conclues avec des compagnies de chemins de fer ou de navigation.

Par ailleurs il importe de ne point s'attarder à des questions de personnes qui, dans tout régime, sont des arguments à côté plus ou moins négligeables. Sous ce nom de factoreries, l'on a pu voir ainsi surgir tout le corps des doctrines économiques se rattachant à la colonisation pratique. Encore un coup, tout se tient, tout est lié dans une partie aussi difficile que celle qui se joue dans ces vastes régions du Congo. Ce n'est pas quand l'essor commercial de la colonie est aussi visible et tangible qu'il convient de ne retenir que des vices qui sont inhérents à un régime transitoire. Celui-ci est destiné à disparaître, quand la mère patrie, dans sa généralité, aura porté ses efforts collectifs et nationaux sur son domaine d'outre-mer. 



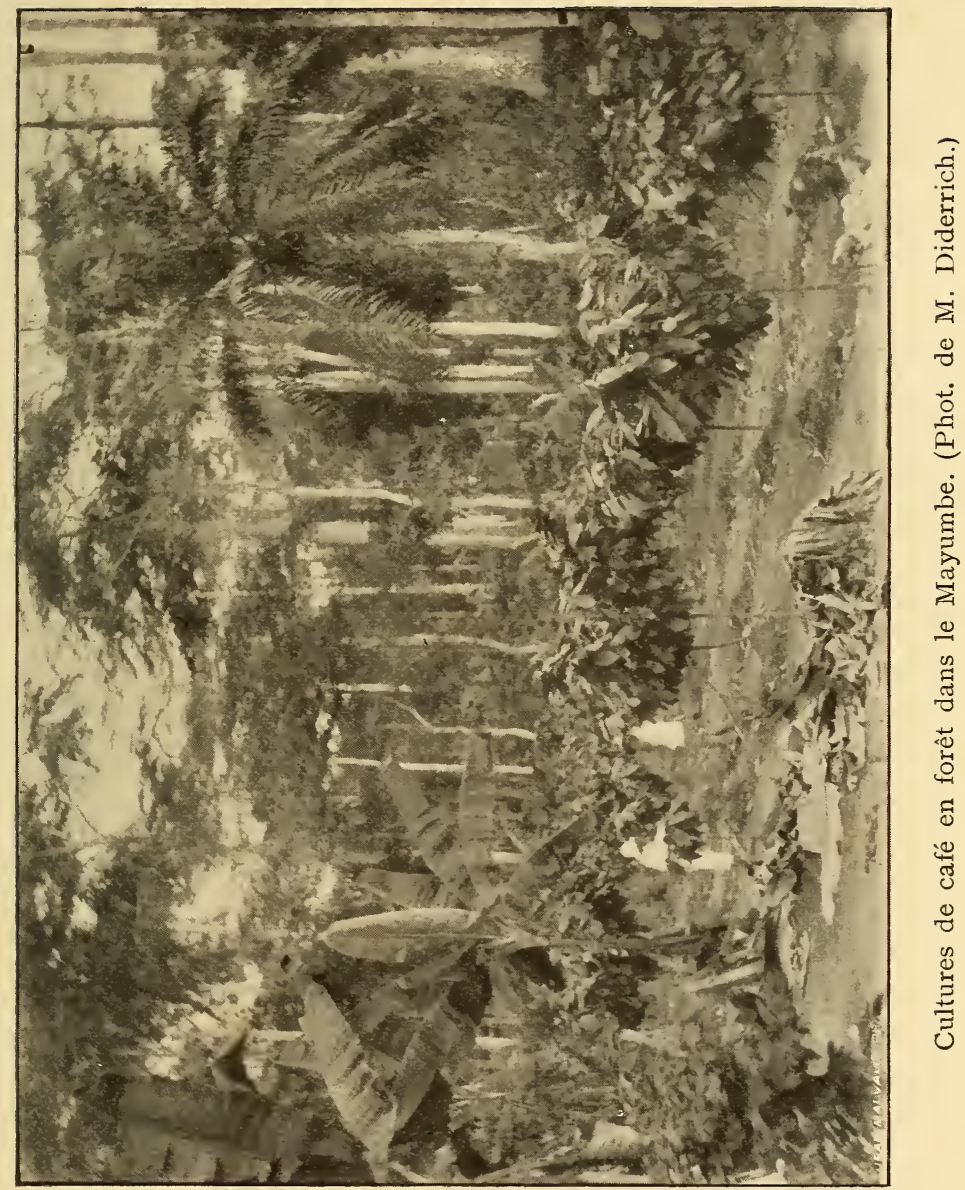





\section{XI}

\section{LES JUGES}

Une séance judiciaire à Léopoldville, dans des formes se rapprochant singulièrement de celles usitées dans notre pays, voilà à coup sûr un spectacle peu banal. C'était le 18 juillet. Le rôle du tribunal siégeant au répressif était chargé de plusieurs causes intéressant des prévenus noirs. L'occasion était on ne peut plus favorable pour saisir sur le vif un coin de réalité africaine, et aussi de psychologie, puisqu'il ne s'agissait de rien moins que de l'application des principes de nos lois de civilisation à des "sauvages ".

L'appareil de la justice, sans connaître les cadres monumentaux dont nous nous sommes plu à l'entourer, 
ne manque pas d'une certaine solennité. La salle d'audience est la plus grande et la plus belle de la localité. Elle est le centre de réunion des blancs, le " mess " et la maison commune en quelque sorte.

Un détachement de la force publique forme à droite et à gauche de la salle, dans le fond, une haie d'honneur en même temps qu'un piquet de garde. A la table, recouverte d'un tapis vert, viennent siéger sur des chaises très simples le juge et le représentant du ministère public. Ils sont revêtus de la robe et de la toque, insignes qui, plus encore que dans nos tribunaux d'Europe, tranchent avec le costume des habitants.

L'audience est ouverte et aussitôt l'on a le sentiment d'une institution jeune en pays neuf.

Le premier prévenu est un Sénégalais, accusé de violences sur la personne d'un Accra. Tous deux sont ouvriers sur les chantiers du chemin de fer. Ils témoignent l'un pour l'autre d'une animosité profonde, le premier se prévalant de sa qualité de sujet français, le second de la nationalité anglaise. Une difficulté va se présenter au sujet de l'emploi des langues. Un agent de factorerie, présent à l'audience et sachant l'anglais, est sur-le-champ assumé comme traducteur juré. Cela complique l'interrogatoire et le fait durer. Cependant très minutieusement, avec beaucoup de patience, le juge recueille les dépositions. Le prévenu nie, le plaignant exagère, les témoins parlent à côté ou autour de l'affaire. Résultat : une peine légère. Le noir Providence est condamné à 26 francs d'amende et aux frais.

Une autre cause, la troisième dans l'ordre des appels, 
est plus intéressante. Il s'agit d'un vol de malles appartenant à un agent blanc. Le délit est grave. Tout d'abord le préjudice est sensible qui vous prive d'effets dont généralement vous n'avez que trop besoin là bas. Ensuite c'est un blanc, qui a donc été molesté par des indigènes. Enfin, il a fallu combiner le coup à plusieurs, car les objets emportés étaient pesants. En fait on a appréhendé cinq individus qui ont agit de concert, cela paraît évident. Point digne d'être noté, ils représentent visiblement parmi les autres noirs, notamment à côté des beaux hommes de la police, des dégénérés.

L'un des prévenus est un affreux petit bonhomme, au facies simiesque, aux membres grêles; c'est un enfant encore. Confondu par un témoignage précis, il ne peut plus nier. Mais trop faible pour emporter au loin la malle qu'on l'a vu tirer sur le sol, pour cacher dans un coin de la brousse des objets volés, il a dû avoir des complices pour terminer l'ouvrage. Il les a dénoncés. L'un a l'air hébété, un autre au contraire roule des yeux rusés et inquiets. C'est lui qui, avec le plus d'effronterie et de loquacité, soutiendra tantôt qu'il ne connaît rien de l'affaire, que l'enfant seul a volé. Malheureusement pour lui, certains des coprévenus, moins adroits, sont en aveu; ils le désignent comme l'auteur de la combinaison, et tout cela rappelle extraordinairement des scènes de nos prétoires. Quand donc les accusés ne se chargent-ils pas réciproquement?

Pour arriver à la découverte de la vérité, il faut encore plus de conscience que précédemment. Ces indigènes, dont deux ou trois viennent du Haut, ne com- 
prennent pas tous le même langage. Un noir servira cette fois-ci d'interprète. Le plus qualifié est un boy du commissaire général; le serviteur du blanc est de par ses fonctions supérieur à ses congénères. Il a de l'acquis, du vernis même, le sentiment de son importance, presque de son autorité. Quand le chef de bande cherche à intimider du regard le plus jeune des prévenus, et que le juge a prononcé les paroles: "Sele, tournez la tête ", Sita, d'un geste énergique, remet la dite tête dans la bonne direction, comme s'il avait à visser ou à dévisser un écrou.

Les traductions sont-elles absolument fidèles? Peutêtre autant qu'elles le seraient en Europe, dans des pays simplement bilingues. Prévenus et témoins comprennent-ils exactement la portée des actes dont on leur demande compte? Estiment-ils à leur valeur ces constants rappels à la vérité que, très consciencieux, le juge leur prodigue? Il n'en faudrait point jurer. Mais de façon rigoureuse et absolue où en est-il ainsi? La chose essentielle, c'est qu'aux yeux du justiciable le magistrat soit doué de la réputation voulue de science, de pénétration, d'impartialité. Les textes des codes ne sont que paroles obscures pour le vulgaire. Il suffit qu'ils représentent la vérité.

Pour le nègre, l'homme blanc est en règle générale l'être d'essence supérieure, plein d'habileté et de sagesse, à ce point qu'ils le choisiront volontiers comme arbitre de leurs propres différends. Bien entendu ils ne s'arrêtent plus à la qualité extérieure, à la fonction du juge considéré comme tel. C'est le chef auquel ils s'adressent et le chef qui aura le plus de prestige, c'est celui qui 
détient la part la plus importante de commandement, que ce soit comme officier, administrateur, fonctionnaire proprement dit, peu importe! Ainsi l'on revient à cette notion couramment éprouvée en pays neuf : "Tant vaut l'homme, tant vaut l'institution. ) Étant d'ailleurs admis que "l'arsenal de nos lois " ne doit fournir que le strict nécessaire aux besoins de réformation d'une société primitive, encore est-il avéré que tels ordres ou défenses de nos codes ne sont applicables que dans la mesure où ils émanent d'un pouvoir visible, concret, en chair et en os, serait-on tenté de dire. Les principes ne viennent que bien après dans l'ordre des raisonnements que peut faire l'indigène. Et cela est très heureux. Quand une société organisée est ancrée dans certaines idées, les individus qui la composent cessent d'être malléables dès qu'on les soumet à de brusques changements. Ils ont leur plan d'évolution à eux qu'il importe de respecter. Cela est vrai notamment pour les peuples de civilisation orientale, à notre sens, stationnaires et arriérés, mais arrivés néanmoins à une grande maturité. $\mathrm{Au}$ contraire, la masse noire est surtout inerte, c'està-dire sujette à recevoir des impulsions. Par l'intermédiaire des rouages judiciaires, il est permis d'agir très efficacement sur elle et c'est ce que fera ressortir encore l'examen, poursuivi d'un peu plus près, des institutions en vigueur au Congo.

" Aller devant le juge ") est une expression que maintes fois vous trouverez sur les lèvres des noirs. Et ils pratiquent la chose.

Massamba, mon boy, - Petro dans le service, - me 
pria d'appuyer auprès du juge une requête en mariage. Il a une compagne, une jeune femme de Djabir, appelée Tosi, qui l'a accompagné dans tout le voyage du "Haut ». Ce qu'il veut, c'est la consécration, par un acte solennel, par l'inscription à l'état civil, d'une union consommée à la mode indigène. Les conjoints vivaient ensemble depuis plusieurs années, l'un et l'autre employés de diverses manières par l'État. Ils paraissaient s'entendre à merveille. Sur le bateau on les voyait constamment réunis. Le soir venu, Massamba dressait la tente commune : quelques nattes placées à même le sol et surmontées d'autres nattes disposées en cintre pour former toiture. Tosi allumait le feu, faisait cuire les aliments ; jamais de dispute ni de violences. Aussi, quand j'interrogeai Jean, le boy du Dr Étienne et camarade de Massamba, sur les motifs de la démarche de mon serviteur, il put répondre avec toutes les apparences de la sincérité : "Lui dire aimer beaucoup femme. " J'étais tenté de penser : "Fort bien, qu'il continue. " Mais en quoi le mariage à l'européenne lui paraît-il à cet effet préférable à l'autre? Ce n'est que plus tard que j'eus le mot de cette petite énigme.

Tosi allait arriver au terme de son contrat de travail (de sept ans). Le cas échéant, elle aurait été rapatriée, et alors c'était la séparation, ou bien elle aurait dû signer un nouvel engagement avec l'État - et cette perspective la séduisait fort peu - ou enfin elle serait entrée au. service d'un employeur blanc quelconque. Nulle part, dans les stations du Congo, on ne peut admettre qu'il y ait des bouches inutiles à nourrir, des bras inemployés. 
Bref! au point de vue de la continuation de leur existence commune, pour Massamba et Tosi beaucoup dincertitude et peu de sécurité. Au contraire, le mariage régulier donne, du consentement des autorités, loisir à la femme de se consacrer presque exclusivement à son ménage. C'est la prime accordée à la vie familiale, à la monogamie. L'épouse est protégée contre les entreprises de qui que ce soit, et l'époux possède une femme dont rien ne peut en fait comme en droit le séparer. Qu'on prenne comme motif déterminant de la conduite de Massamba et Tosi, l'attachement réel de deux cœurs et pourquoi serait-ce impossible? - ou l'intérêt, toujours est-il que dans l'un et l'autre cas le désir d'établissement était curieux à noter; et il n'était pas moins intéressant d'observer que c'est dans l'intervention du juge, dans la protection accordée par le représentant de l'autorité légale, que mon boy voyait le meilleur moyen d'arriver à ses fins. Je fis donc ce qu'il me demanda. Je remis une mukande (le certificat avec avis approbatif) au procureur d'Étatà Boma, qui me promit une solution favorable. Serait-il vrai que j'ai préparé une idylle africaine? Philémon et Beaucis, où êtes-vous?...

Ces recours à la justice et à la protection du blanc sont directement favorisés par l'organisation même du pouvoir judiciaire. Aux différents agents du ministère public incombent spécialement les devoirs et les charges d'une sorte de tutelle qui s'étend sur la masse des indigènes considérés comme des mineurs. De cette tutelle relèvent les mesures d'intervention relatives au contrat de travail, aux aliénations foncières consenties par les 
indigènes, aux engagements qui intéressent soit leurs personnes, soit leurs biens. Les rapports sont encore limités entre civilisés et sauvages. Mais quand ils existent, ils engendrent aussitôt des droits certains au profit des plus faibles, c'est-à-dire des derniers.

Les noirs en ont si bien conscience qu'ils ne se font pas faute de porter plainte contre les hommes blancs qui les molestent. Il aura notamment tout lieu deregretter son acte ou " son geste " le maître qui, cédant à un mouvement intempestif de vivacité, aura maltraité son serviteur. La contre-partie de cette juste sévérité, c'est l'obligation pour l'employé de rester fidèle à son engagement, sous peine d'un châtiment sévère. Le coupable qui est puni accepte d'ailleurs avec assez de philosophie les pénalités prononcées. Le tout est de frapper juste.

Une fraction minime des indigènes gravite dans l'orbite tracée par notre action civilisatrice. Le plus grand nombre reste jusqu'ici soumis à ses propres mœurs et coutumes, sous réserve des contraintes et des prohibitions fondamentales qu'entraîne immédiatement l'arrivée des Européens. Nulle part, cela va de soi, l'anthropophagie, les exécutions sanglantes, la traite, la guerre intestine ne sont permises. La répression alors n'a d'autres limites que les difficultés matérielles qui s'opposent à son exercice. Le principe est que tout natif peut se mettre sous la protection des lois de l'Etat. Jamais celle-ci ne lui sera refusée sous prétexte qu'elle se mettrait en contradiction avec les lois de la peuplade ou de la tribu. Il arrive même que, pour le règlement de leurs propres différends, les indigènes viennent de fort 
loin se soumettre à la juridiction gracieuse des blancs. C'est le chef le plus élevé en grade et en autorité qui est investi du rôle d'arbitre. Son prestige s'augmentera de toute la sagesse de ses décisions. A cet effet, il doit s'armer d'une patience à toutes épreuves. Les plaideurs, eux, ont tout le temps, et leur éloquence est intarissable. Pour des procès de ce genre, chaque partie - du moins la chose se passait ainsi dans la région des Cataractes apporte des épices, des têtes de bétail, par exemple. L'équité veut souvent que les fautes et les responsabilités soient partagées. Les deux plateaux de la balance se garnissent ainsi de manière appréciable, tout en restant le symbole de la justice la plus impartiale

On le voit, l'idée de justice obéit, dans le milieu congolais, à des impulsions diverses. Les unes ouvrent comme des percées dans la brousse épaisse de la sauvagerie, d'autres se rattachent de façon plus intime à ladministration judiciaire proprement dite. Dans ce mélange encore un peu inorganique il y a place pour des conflits d'attributions assez sérieux. Tant qu'on est dans le Bas-Congo, à Boma, à Matadi, même à Léopoldville, c'est-à-dire dans les centres les plus avancés, en possession des organes essentiels de gouvernement qui se combinent et s'harmonisent avec flus ou moins d'équilibre, les difficultés ne se présentent guère. Le tribunal d'appel, le tribunal de première instance de Boma fonctionnent approximativement comme les cours et les tribunaux d'Europe. La séparation ou plutôt l'indépendance absolue des pouvoirs, si elle n'existe pas encore complètement, est en passe de s'y instaurer. 
Mais dans le Haut-Congo, au sein de la forêt équatoriale, ces principes sont d'application plus malaisée. L'organisation judiciaire - et il n'en peut être autrement - est encore ici à sa phase préparatoire. Sous le nom de tribunal territorial, dans chacun des districts les plus importants doit siéger un collège composé d'un juge, d'un substitut et d'un greffier. Le plus souvent le juge n'est pas entré en fonctions; seul le substitut, qui représente le contrôle du pouvoir central, l'action publique si l'on veut, est là avec mission de faire respecter les lois ainsi que les règles d'humanité en général. Pour appeler les choses par leur nom, il doit incarner la légalité, à côté des agents d'exécution que sont les commissaires de district, qu'ils soient chefs militaires, administrateurs politiques, entrepreneurs de colonisation et d'exploitation au sens élevé du mot.

Tout d'abord il y a disproportion évidente entre les hommes - tenons-nous à des types généraux investis respectivement de ces fonctions. L'un, "le juge ", pour l'appeler de son nom générique, est jeune, plus ou moins inexpérimenté, dans une situation matérielle très modeste, tant à raison de l'exiguïté de son traitement qu'à raison de la dépendance où il se trouve, pour les détails quotidiens de son existence, - logement, nourriture, ravitaillement, — vis-à-vis du commandant de district.

Celui-ci, au contraire, est, dans le langage courant, un big chief, un grand chef, un homme considérable et digne d'être considéré, qui a fait ses preuves, en expédition, en exploration, en palabres avec les indigènes, qui 
peut tenir un certain train grâce à des appointements considérables, qui, pour avoir risqué sa vie, en sait le prix, et qui dans l'exercice de responsabilités souvent énormes, a gagné jusqu'à l'hypertrophie le sentiment du commandement et de l'autorité.

Imaginez maintenant un conflit entre ces deux hommes, et mettez qu'il ne soit que de nature théorique. Si le juge est conscient de sa situation, il cédera, même quand il a raison. Ou bien, versant dans l'excès contraire, il n'aura que le souci d'affirmer à tout bout de champ l'autonomie du pouvoir qu'il incarne. Le commissaire de district, quant à lui, par tempérament peu sympathique à la gent de robe, sera en perpétuelle défiance à l'égard du juge le plus modéré et le mieux disposé en réalité. Il verra en lui un censeur importun, pour ne pas dire un espion. Et voilà le conflit organisé, pour mettre les choses au pire.

Quel remède conviendrait-il de suggérer à cette situation?

A vrai dire, le plus radical consisterait à supprimer la cause même des conflits. En pays neuf il est inopportun de pousser à l'extrême le principe de la séparation des pouvoirs. De fait, c'est l'homme d'action, celui qui fait le plus, qui doit l'emporter. Que le commissaire de district reste investi de la plénitude des pouvoirs judiciaires, nous ne verrions certes rien à y contredire. Le correctif, c'est l'entièreté des responsabilités. Pour prémunir l'administrateur contre les risques d'erreurs juridiques, il suffit de le doubler d'un conseiller légal, mais celui-ci bien entendu dépourvu de tous pouvoirs 
propres. Il avertit et renseigne, il rédige au besoin, mais il n'a pas à se mettre en opposition avec son chef hiérarchique $\mathrm{T}$ el, chez nous, le greffier du tribunal de commerce.

C'est pour le coup, objectera-t-on, qu'il n'y aura plus aucun frein à l'arbitraire éventuel des agents de l'exécutif. En est-on si certain? Rien n'empêche d'organiser un contrôle judiciaire infinement plus étendu que celui qui existe actuellement au Congo. Après avoir suivi les Anglais dans la très grande liberté d'allures qu'ils affectent au regard du dogme de la séparation des pouvoirs, - surtout aux colonies, - pourquoi ne prendrions-nous pas modèle sur leur institution des juges itinérants?

$\mathrm{Au}$ lieu que les affaires viennent se centraliser à Boma, après des retards et des détours considérables, qui peuvent aller jusqu'à les dénaturer complètement, il vaudrait sans doute mieux que les juges de Boma aillent par délégation s'enquérir sur place des affaires du HautCongo. Des cours de justice se tiendraient successivement dans les chefs-lieux de district, pour toutes les affaires assez importantes pour être évoquées et instruites devant elles. Entre le commissaire de district et ce vrai juge, - un grand juge, si l'on veut, - il ne se sera pas produit ces frictions inséparables d'un commerce quotidien. L'homme de loi apparaîtra avec la dignité et l'autorité que lui conféreront sa situation, son expérience, son indépendance. En d'autres termes, c'est l'inspection judiciaire qui viendrait se substituer à lintervention incessante de tous les jours. Le moindre 
mérite du système pourrait par ailleurs n'être pas de mettre en face des cas difficiles et sur place les hommes qui, jusqu'à présent, n'ont pas à donner leur mesure dans le Bas-Congo où, répétons-le, tout est réglé normalement et, d'autre part, de décharger des juristes insuffisamment préparés de tâches que l'éloignement, le manque de points d'appuis rendent précisément des plus délicates.

Les détails d'organisation sont d'essence très variable. Ce n'est pas le moment de les discuter. Le respect dû à l'idée de justice, en même temps que la nécessité de laisser la plus grande somme d'autorité possible aux agents locaux du pouvoir exécutif, sont des idées fondamentales auxquelles, assurément, il était permis de s'attacher dans l'examen des institutions judiciaires déjà si remarquables de l'État Indépendant. 



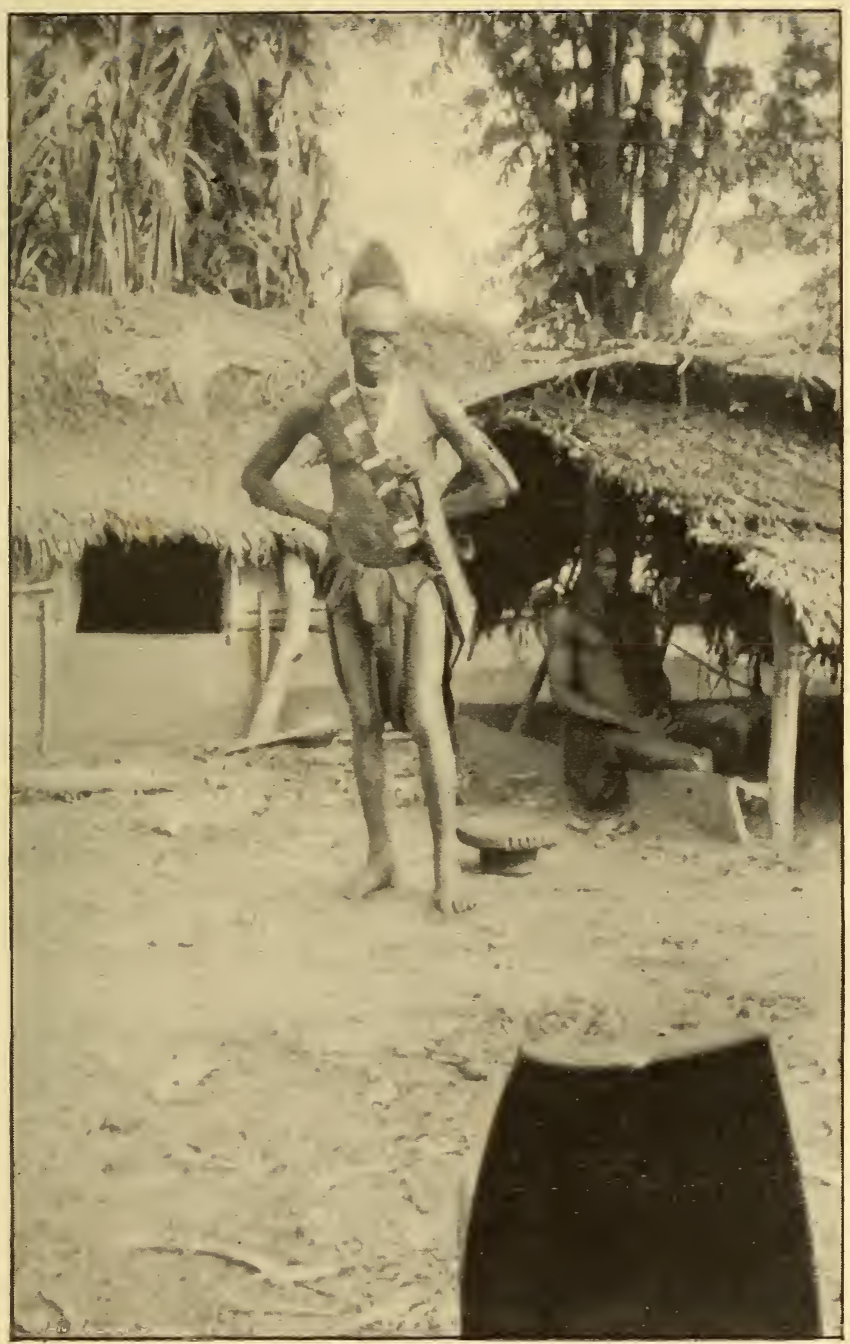

Le chef Mokoko. 



\section{XII}

\section{NOS FRÈRES NOIRS}

Au Congo, comme dans toute l'Afrique tropicale du reste, c'est à vrai dire la question préalable qu'il faut opposer aux protagonistes de la théorie de la disparition fatale des races inférieures. En effet, avant même de discuter ce qu'il y a souvent de spécieux et de superficiel, en même temps que de brutal, dans les condamnations sommaires portées, au nom du principe commode de la lutte pour l'existence, contre telle ou telle fraction de l'humanité, il importe de tenir compte de ce fait primordial que, sans les noirs, il n'y a rien à faire dans la plus grande partie de l'Afrique. Les blancs ne pouvant espérer d'ici longtemps, sinon jamais, s'y acclimater, 
c'est-à-dire se fixer définitivement au sol, et fonder des familles, leurs sentiments philanthropiques se concilient avec leur intérêt bien entendu, ce qui est encore le plus sûr moyen d'amener entre eux un accord harmonieux. La possession de la terre arable, en dehors des violences presque inévitables de la conquête, a été la cause principale des exécutions en masse et systématiques auxquelles ont procédé les colons de l'Amérique du Nord et de l'Australie à l'égard des populations indigènes. Il n'y a pas place en une région déterminée, à la fois pour un chasseur qui a besoin d'espaces illimités, et un agriculteur qui s'emploie à mettre en valeur chaque parcelle du sol. Le conflit ne peut être évité que si le sédentaire parvient à gagner le nomade à sa cause ou tout au moins si celui-ci se soumet à la loi du plus fort. Les Anglo-Saxons n'ont souvent eu qu'un médiocre souci des moyens de persuasion. C'est par là qu'ils ont péché, qu'ils ont imprimé une tare indélébile à leur expansion coloniale.

L'extermination, en Afrique, ne ferait que des vides, des vides qu'il serait impossible de combler par l'afflux d'immigrants venant des pays plus avancés en civilisation. Du moment que la coexistence d'éléments ethniques différents est reconnue indispensable, et cela en proportions telles que le fond indigène soit comme le tronc de l'arbre que recouvre un peu de mousse seulement, les quelques Européens, - une attention toute particulière doit être vouée au travail d'association, à la meilleure combinaison de ces éléments. Quand on est bien conscient de la tâche à remplir, on est beaucoup plus 
près de l'aborder par le bon côté. Les missionnaires diront volontiers que, pour réussir, il faut être animé de l'esprit de charité. Mieux que tant d'autres ils se rendent compte de la mentalité arriérée, des instincts fâcheux des noirs Ils les appellent cependant des frères, ils les convient au banquet spirituel d'idées, de sentiments et de croyances qui sont échus en partage aux nations les plus policées de l'ancien monde. Et dans cette égalité morale proclamée à l'encontre de la visible inégalité matérielle, il y a assurément une grande, une très grande force.

Le prosélytisme est une chose, la politique en est une autre. Pourtant, en prenant comme point de départ cette puissance d'expansion inhérente à la civilisation occidentale ou chrétienne, ne pouvons-nous admettre que le don de sympathie et, si l'on veut, d'assimilation bien comprise ait à jouer son rôle dans la colonisation?

Qu'on ne croie pas à de vaines illusions, à des rêves plus ou moins chimériques. D'éclatants exemples attestent la possibilité d'allier, dans ce domaine, le bon sens le plus ferme aux vues les plus généreuses. Livingstone, le plus illustre et le plus éclairé des explorateurs, aima sincèrement les noirs au milieu desquels il vécut tant d'années de sa noble existence; Gordon, aux initiatives si hardies, à la fois diplomate et conquérant, sut se dévouer corps et âme à la répression de la traite soudanaise, sous laquelle il souffrait de voir périr des populations entières. Prenez encore des hommes comme Faidherbe, Savorgnan de Brazza, Emin Pacha, ceux que leur autorité, leur situation, leur science mettent 
certes fort au-dessus du commun, chez tous vous observez une indulgence pleine de bienveillance à l'égard des noirs. Ils les trouvent constamment dignes d'intérêt, susceptibles de progresser, de se développer.

Assurément, ce n'est point au cours d'une rapide excursion que j'ai pu rassembler les éléments d'une psychologie congolaise. N'empêche que dans le commerce quotidien avec gens de l'équipage, passagers, soldats, avec toute la smalah qu'est la population d'un bateau sur le haut fleuve, dans les mille incidents qui accompagnaient nos brefs séjours à la rive, bien des traits de vie affective s'offraient à notre observation, sans compter ceux qui nous étaient rapportés sur place par les blancs étabfis dans les différentes stations.

Les scènes d'adieu ou de bienvenue aux arrêts du bateau rappelaient à s'y méprendre celles qui se produisent en pareille occurrence dans nos voyages d'Europe. Démonstrations plus ou moins exubérantes, émotion de ceux qui se séparent, rien ne manque parfois au tableau qui surgit pittoresque ou touchant.

Le barreur Lulongo, originaire d'un village de la rive gauche, de la région des Bangala, est arrivé au pays. Il a revêtu ses plus beaux vêtements, une chemise propre, une vieille jaquette de marin ornée de boutons jadis dorés. En grande hâte il est descendu à terre. Profitant de la halte au poste de bois du fleuve, qui devait se prolonger plus que de coutume, je me promenais un peu au hasard. Suivant un sentier tracé dans les hautes herbes, après une petite demi-heure de marche, je débouche dans un village indigène. Quelle ne fut pas 
ma surprise d'apercevoir bientôt, auprès d'une des cases, le barreur Lulongo jouant de l'accordéon devant la famille assemblée. Grands et petits faisaient cercle. C'était fête à l'occasion de la visite du voyageur. Pour un peu le barreur me présentait aux siens. Il leur disait de me tendre la main. Mais les plus petits, en voyant un blanc, se mirent à crier d'effroi

Chose étrange, quand on sait qu'on a, en sa présence, des cannibales, - de penchant du moins, - leur physionomie ne respire généralement pas la férocité à laquelle on s'attend. Chez les plus âgés, l'expression trahit bien quelque défiance, avec une nuance d'hostilité déguisée, que rend plus désagréable encore le facies assez abruti, presque bestial, défiguré par de monstrueux tatouages. A mesure que s'affirme plus nettement la jeunesse, le visage a des aspects rieurs de grand enfant insouciant. Quant aux bambins, ils sont parfois charmants de vivacité. Beaucoup plus précoces que les nôtres, - à sept ou huit ans, un boy est un petit homme plein de ressource et d'adresse, - ils laisseraient à penser que leur intelligence vaut et surpasse celle des blancs.

C'est ainsi encore qu'à l'école le négrillon apprendra à lire plus vite que ne le pourrait un petit paysan de nos campagnes. Seulement avant l'âge adulte même, il se produirait comme un arrêt de développement dans la mentalité de l'indigène. Le fait n'est guère douteux dans le milieu autochtone. L'usure physique, pour des causes imparfaitement déterminées, - peut-être des unions prématurées, - est rapide. L'homme mûr, et certes le vieillard, si tant est que la vieillesse soit 
atteinte, est un être déchu, qui appelle la dérision, plutôt qu'il ne commande le respect. Avec des modifications dans la manière de vivre, avec des habitudes familiales de moralité plus rigoureuse, peut-être la situation s'améliorera-t-elle. C'est ainsi qu'on est constamment ramené à la question d'éducation, à la pénétration de l'esprit et du cœur du noir.

A cette fin, il faut commencer par le comprendre, ceci dit au sens littéral du mot. Pour d'aucuns, au jugement de qui il n'existe que le " sale nègre ", il suffit d'un vocabulaire restreint, une sorte de sabir commercial, pour entrer en relations avec les indigènes. Ceux-ci n'ayant que des besoins très limités, en harmonie avec une manière d'existence végétative, n'éprouveraient que le minimum, on dirait bien, de sensations. Pour l'expression de sentiments aussi rudimentaires, trois cents mots sont plus qu'il ne faut. Ainsi, me disait-on, le terme aimer est inconnu des noirs. Boutade ou non, l'appréciation ainsi formulée m'apparut aussi hasardée que dangereuse. J'en acquis bientôt la preuve en deux circonstances qui constituent de vraies leçons de choses et qui à ce titre valent la peine d'être rapportées.

Les faits sont, en dernière analyse, l'argument décisif qui doit emporter la conviction.

A Mosembi, dans la région des Bangala, j'eus l'occasion de m'enquérir auprès de $M$. Weeks, missionnaire anglais, du bien ou du mal fondé de l'assertion relative à la pauvreté de la langue indigène. Il travaillait précisément - et dix-sept ans d'Afrique lui donnaient sous ce rapport une compétence certaine - à la constitution d'un 


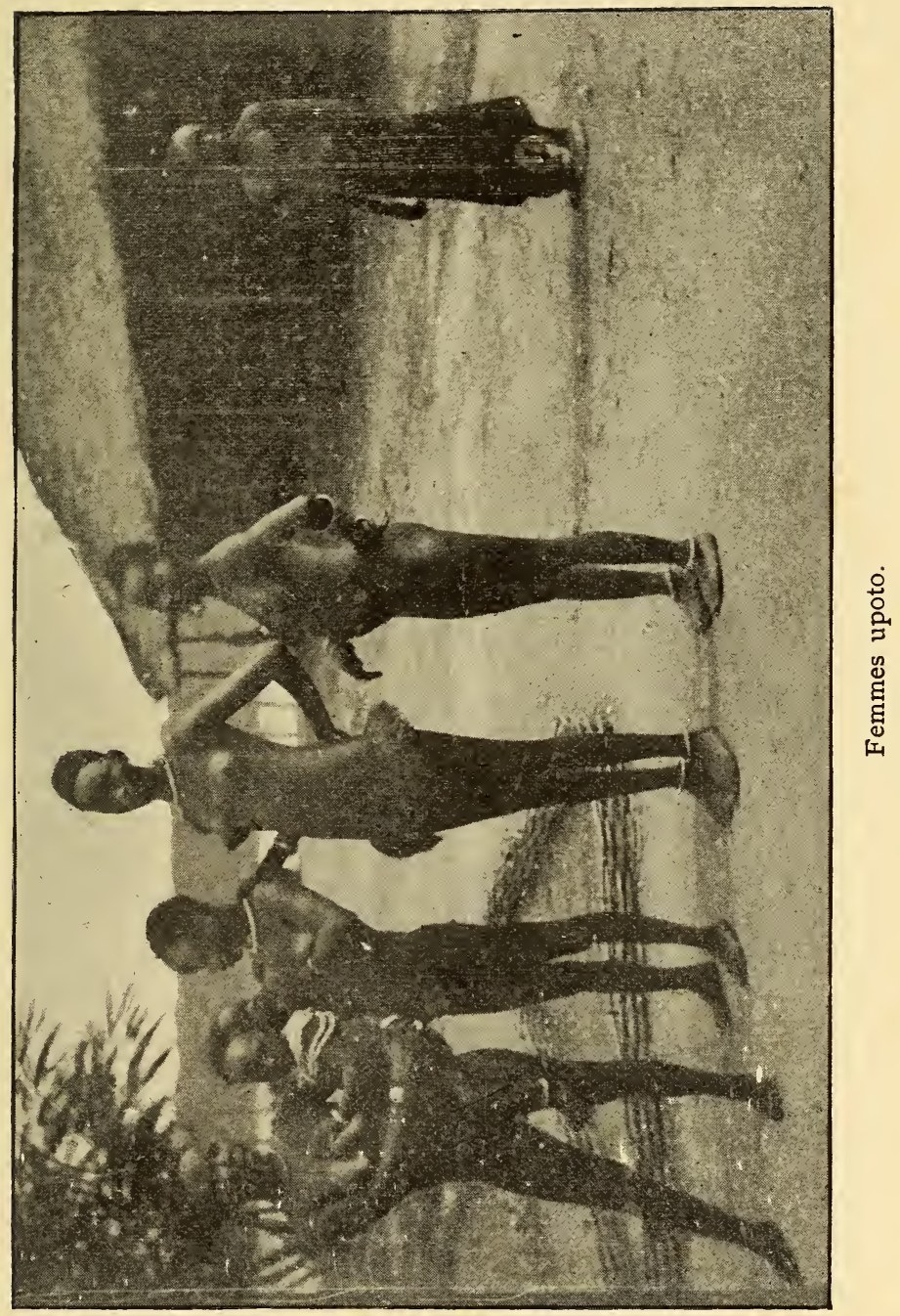



lexique et d'une grammaire bangala. Pour le premier il avait recueilli d'ores et déja douze mille mots sur un total qui, à son estime, devait s'élever à vingt-cinq mille. Quant à la grammaire, elle serait particulièrement riche en flexions et en nuances. Voilà, prouvée de la façon la plus irrécusable, la supériorité que confère à l'homme instruit, sur l'individu resté inculte, la préparation scientifique et intellectuelle. Pour comprendre le sauvage, il n'est peut-être rien de mieux que d'être soimême très civilisé. Tel esprit aiguisé et raffiné saisira mieux les bégaiements moraux d'une société primitive, au stade de l'enfance, que ne le pourra telle intelligence trop fruste, de jugement sommaire et un peu brutale.

Nous parlions, il y a un instant, des Bangala. Comme on le sait, l'équipage de la Princesse Clémentine en comprenait un assez fort contingent : barreurs, mariniers, chauffeurs. Il advint un jour que, dans l'encombrement du pont inférieur, une femme trébucha et tomba à l'eau. Elle nageait juste assez pour se soutenir à flot. Avant de stopper et de faire machine en arrière, le bateau, par la vitesse acquise, avait pris sur la pauvre négresse une avance relativement considérable. Livrée à ses seules forces, elle aurait vraisemblablement péri. Mais voilà que d'un bond un homme, un noir taillé en hercule, n'écoutant que son courage, - car il en faut dans un fleuve infesté de crocodiles, - se jette à l'eau. A grandes brassées il a bientôt fait de rejoindre la femme; la soutenant, il réussit à la ramener saine et sauve à bord. Le sauveteur était le mari. Le lien d'affection qui l'unissait à la femme était donc assez fort pour 
que lui seul tentât ce qu'aucun autre ne fit en face du danger. Ce n'est pas tout. Pour recueillir la sauvée, il y avait à bord une autre femme, plus âgée que la première. Les deux créatures s'enlacèrent étroitement, et pendant une demi-heure, sans dire mot, pleurèrent des larmes d'émotion et de joie. C'étaient la mère et la fille. Elles ne prononçaient pas le verbe aimer, elles le ressentaient

Nombreux sont d'ailleurs les " cas passionnels " que nous rapportèrent les blancs établis au Congo. Jalous:e comme amour, sinon fidélité et respect des liens conjugaux, se rencontrent fréquemment.

A Stanleyville, M. le commissaire de district Malfeyt nous parla d'un gendarme, que ses disgrâces maritales conduisirent, en désespoir de cause, au suicide. Ailleurs, des fonctionnaires nous dirent tous les soins dont ils furent l'objet de leurs serviteurs, alors qu'ils étaient malades. Des officiers de troupe nous racontèrent les traits de dévouement, de sacrifice personnel jusqu'à la mort, consentis par certains de leurs subordonnés, dans des moments critiques et périlleux en campagne. Tous ces faits mériteraient d'être colligés pour être, le cas échéant, rappelés à ceux qui font trop bon marché de la personnalité humaine chez les habitants de l'Afrique centrale.

Dans cet ordre d'idées, il est bon de se tenir à égale distance de deux opinions qui, prenant un point de départ distinct, aboutissent néanmoins à des conséquences pareillement fâcheuses.

Dans l'une le nègre est cette brute inconsciente, dont 
la force musculaire au plus est bonne à employer. Sa nature foncière, au contraire, serait à jamais réfractaire à toute idée élevée, à tout progrès moral. L'éducation à lui conférer devient du dressage, comme on l'appliquerait à un animal supérieur, mais ne ressemble ni de loin ni de près à l'œuvre de relèvement que seuls peuvent concevoir des philanthropes à l'esprit chimérique. Cette théorie, qu'on le veuille ou non, aboutit à la destruction de la race noire, car on ne priverait pas impunément toute une fraction de l'humanité du droit à l'idéal. Les idées sont la force et la vie des peuples comme de l'individu. Partant elle doit être condamnée et rejetée.

Dans l'autre opinion le nègre est simplement représenté comme ayant une mentalité si à part, qu'elle n'est pas susceptible de recevoir les empreintes diverses de la civilisation occidentale. En conséquence, il faut pousser jusqu'aux plus extrêmes limites le respect de la manière d'être des indigènes, ne contrarier en rien le plan d'évolution qui leur est propre. Cette doctrine, sous des dehors spécieux, et tout en contenant une part de vérité, renferme des germes redoutables d'erreur. Elle dissocie jusqu'à l'exagération des éléments qui, en réalité, se prêtent à des combinaisons variées

Historiquement elle a le tort de confondre les noirs avec les Asiatiques qui, eux, suivent effectivement des voies parallèles à celles qui nous sont ouvertes et destinées, en conséquence, à ne jamais se rencontrer avec les nôtres. Elle ne tient pas compte du fait que, repliée sur elle-même, réduite exclusivement à ses énergies propres, la race noire risque fort de ne point 
dépasser le niveau qu'elle a péniblement atteint Avec le temps la différenciation n'ira que s'accentuant entre blancs et noirs, de sorte que d'opposition en opposition on arrivera encore une fois à la lutte violente, à l'exploitation du faible par le fort, à l'arrêt de mort prononcé contre le vaincu.

N'y a-t-il pas lieu plutôt de croire à la vertu efficiente et quasi universelle de certains de nos principes de civilisation? Non pas qu'il faille transplanter au Congo nos lois, imposer nos mœurs, nos croyances, nos exigences sociales à qui ne veut pas les accepter et qui, le voulûtil, ne le pourrait pas. Cette méthode d'assimilation ne souffre pas l'examen. Plus personne ne la préconise. Mais de même que dans la nature on greffe un plant dû à une culture perfectionnée sur un sauvageon afin de redresser celui-ci et de l'améliorer, de même il est possible d'inculquer aux noirs quelques notions supérieures, principes actifs destinés à les sortir de leur apathie originaire, à prévenir les tristes écarts de leur tempérament de "sauvage ". Ou bien encore, pour recourir à une autre comparaison, n'est-il pas désirable d'introduire dans le milieu indigène quelques ferments qui fassent comme lever toute cette pâte numaire demeurée inerte jusque-là.

Dans toute l'Afrique, et au Congo notamment, en dehors même des mélanges de sangs, des interventions extérieures se sont produites par le fait des Asiatiques et de l'influence arabe. Va-t-on a priori arguer d'incapacité et de stérilité toute initiative de même nature parce qu'européenne. Ainsi posée, la question mérite au moins 
l'attention éclairée de tous les peuples colonisateurs. Au Congo, la solution en est cherchée expérimentalement. Des résultats partiels, nous l'avons vu, sont acquis. Il importe que ces expériences soient poursuivies avec un redoublement de vigilance et de confiance. 



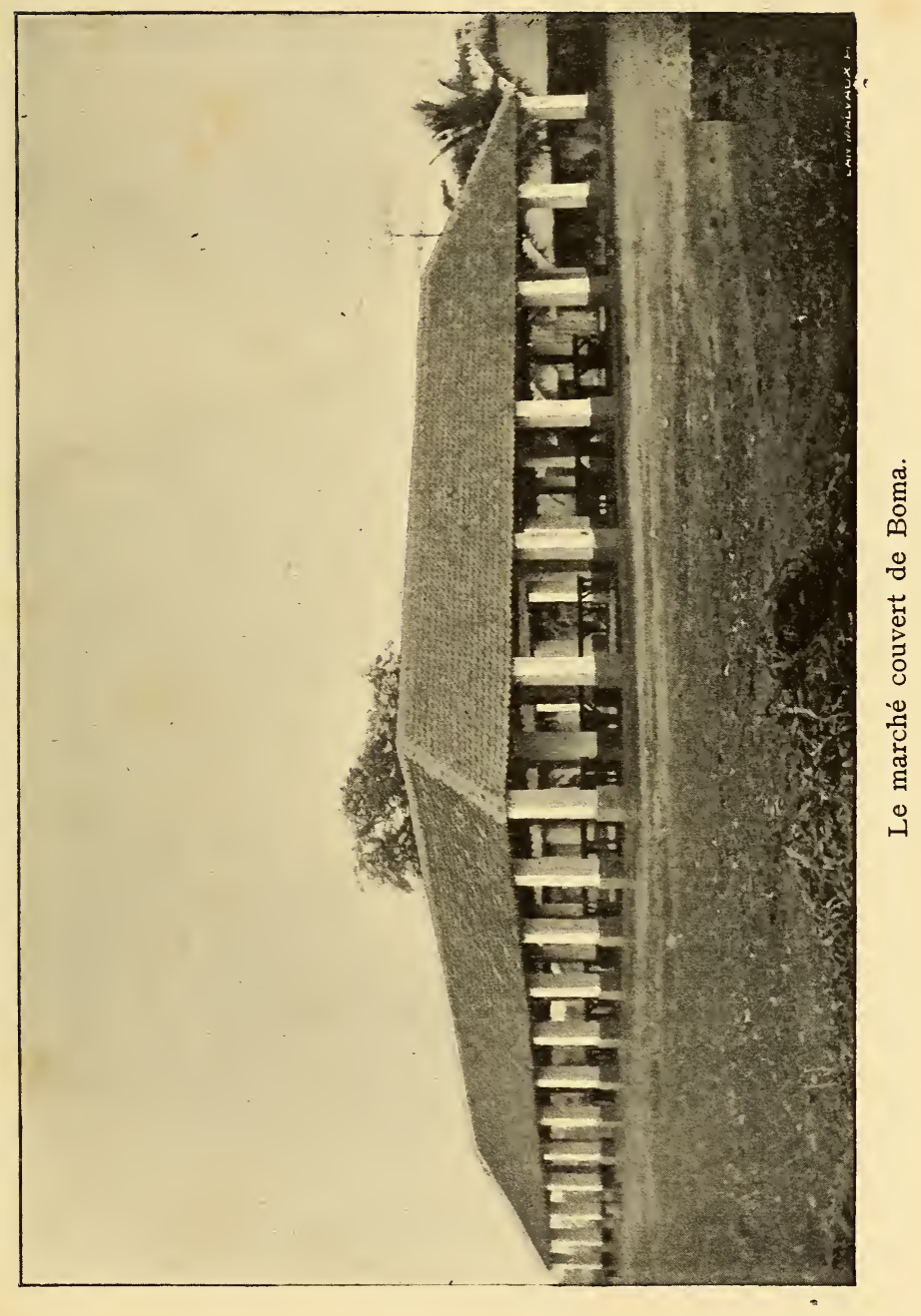





\section{XIII}

\section{QUELQUES CONCLUSIONS DE VOYAGE}

En peu de mois, sur le haut fleuve, il m'a été donné de suivre une route qui est comme bordée de constructions intéressantes. Et si l'on peut dire que le simple touriste - que je fus - n'en aperçoit, en passant, que les façades, il n'est pas moins vrai, quand l'ajustement et l'harmonie en sont bien observées, que le dehors laisse mieux que deviner l'agencement intérieur. Au surplus, les ouvertures ne manquent pas par où plongent sans peine les regards quelque peu curieux.

Les yeux voient, mais ne seraient-ils pas bien médiocres s'ils ne rapportaient pas autre chose au 
cerveau que des images photographiques. Il appartient au contraire à chacun de déduire des faits leur signification intime, de vérifier leur force probante. Ce n'est pas faire preuve d'audace excessive, je pense, de franchir, au figuré comme au propre, tel ou tel seuil, afin de fortifier les impressions extérieures par des réflexions qui seules conduisent à une conviction sérieuse. Je me demande d'ailleurs s'il est nécessaire d'appuyer sur les opinions qui me sont personnelles; ne suffit-il pas qu'à l'aide des choses vues que très sincèrement j'ai rapportées, le lecteur opère à sa manière les rapprochements et les comparaisons indispensables dont je ne me permets, avec une certaine insistance, que de lui suggérer le principe.

De façon générale, sachant ce qu'ils savent d'un passé si récent, et découvrant la situation actuelle, comment les Belges ne seraient-ils pas frappés de la grandeur des résultats obtenus au Congo. En vingt ans, là ou n'existait rien encore de la civilisation, s'élève un édifice colonial aux proportions déjà imposantes.

Dans le bas fleuve, c'est Boma d'abord, ville de près de deux mille âmes, centre d'un gouvernement solidement établi, muni de tous les organes essentiels qui sont l'administration, l'armée, la justice, les postes, les écoles; distributeur aussi de vie économique par son port, son marché et, en dernier lieu, par son chemin de fer de pénétration vers le Mayumbe dont la construction vient d'être commencée.

Un peu en amont, c'est Matadi, dont le nom évoque tant de luttes et de sacrifices, tant d'épreuves victorieuse- 
ment surmontées à force d'énergie et de patience. Le roc s'est changé doublement en port et en gare. Cette transformation, avec la suite grandiose qu'elle comporte, c'est-à-dire le chemin de fer de la région des cataractes, est entre toutes celle qui parle le plus fortement à l'imagination. Le rail devient en quelque sorte le symbole de l'ascendant que prend l'homme civilisé sur la nature sauvage. De fait, privé de cet instrument supérieur, il devait se heurter tôt ou tard, dans son travail de colonisateur, à des difficultés irréductibles. Du jour même où a pris corps l'idée de fonder au Congo un grand établissement, le chemin de fer était donc dans la logique des choses. L'aride région des cataractes, fermée pendant des siècles aux rares factoreries installées aux environs de l'estuaire, ne pouvait cesser d'être - sous le rapport économique - une barrière quasi infranchissable qu'à condition d'être traversée rapidement et aux moindres frais. L'art de l'ingénieur a résolu ce problème ardu qui, à vrai dire, et aussi dure que fût la tache, n'était en aucune façon au-dessus des données présentes de la science.

Le plus laborieux, en cette matière, fut d'inculquer au public la confiance nécessaire dans la validité intrinsèque de l'entreprise, comme aussi la foi dans l'avenir de l'œuvre congolaise considérée en son entier. A cette fin il fallait voir bien au delà du Stanley-Pool, terminus du chemin de fer, il fallait fixer son attention sur la partie la plus mystérieuse de l'Afrique centrale, lui arracher presque de force ses secrets, concevoir et exécuter tout un plan de conquête et d'organisation, avec des moyens 
qu'on serait tenté de qualifier de dérisoires, si le succès n'était venu en fin de compte démontrer qu'ils étaient appropriés à leur objet. A cet égard, le voyage du HautCongo procure une série ininterrompue de surprises réconfortantes. Eh quoi! ce sont des cannibales d'hier, ces riverains soumis, dociles, souvent accueillants que nous avons rencontrés journellement? Ces villes naissantes, ces postes de culture, ces camps militaires, ces factoreries, ces missions religieuses, tout cela a surgi au sein de la brousse inextricable ou de la forêt, par le seul effort de quelques centaines de blancs et des noirs formés à leur discipline? Rien n'est plus certain cependant. Travaux de la paix et de la guerre se sont succédé sans interruption. Les uns et les autres tiennent de l'épopée.

Congolâtre, disait-on jadis, et l'appellation faisait pendant à celle de Kroumir ou de Tonquinois qu'on décernait par dérision aux promoteurs de la politique coloniale chez nos voisins du Midi. Soit dit en passant, l'un des initiateurs les plus méritants de cette politique, Jules Ferry, se voit élever une statue à Tunis pour l'œuvre qui fut décriée avec tant de véhémente passion, avant d'être exaltée avec l'enthousiasme qu'on sait par un peuple reconnaissant.

Aujourd'hui, en tous pays, les coloniaux peuvent revendiquer pour eux le mérite d'avoir su comprendre les premiers la haute portée ainsi que l'avenir prodigieux du mouvement d'expansion qui caractérise, chez les races supérieures de l'Europe, le siècle à son déclin ou plutôt à son tournant d'histoire.

La Belgique fut d'abord hésitante et cela se conçoit. 
Elle a appris du passé à borner ses ambitions. Son territoire exigu, sa population restreinte par rapport à celle de ses puissantes voisines, l'inclinaient à adopter des visées modestes, en rapport avec sa position d'État neutre. Peu à peu, néanmoins, par le prodigieux développement de ses ressources, par son travail et ses entreprises industrielles, couronnés de succès à peine égalés ailleurs, elle a pris conscience de son importance. Économiquement, elle est devenue une grande puissance, une très grande puissance même. Sous peine d'être menacée d'une sorte d'hypertrophie, il lui fallait sortir de son recueillement. Je doute qu'elle l'eût fait de plein gré. Mais à ce moment, traduisant ses besoins, devançant ses aspirations, se fit entendre une voix autorisée entre toutes. Le pays se rendit à ces appels éloquents qui lui ouvraient des horizons nouveaux, les voies coloniales. La pensée initiale de l'entreprise congolaise est celle de notre Roi. Quelque idée qu'on se fasse de ses chances de réussite dans l'avenir, plus personne ne conteste qu'elle ne soit géniale. Elle s'est manifestée par priorité d'invention, peut-on dire; de ce chef, une primauté spéciale lui est assurée. Et, fait non moins remarquable, elle a été suivie sur le terrain même d'expériences pratiques qui sont la pierre de touche des conceptions fortes et originales.

Nous savons ce qu'a été l'exécution. L'intelligence, la vaillance, l'élan dont ont fait preuve les hommes qui se sont dévoués à l'œuvre coloniale ont été un sujet d'étonnement pour le monde et, il faut le reconnaître à notre confusion, pour nous aussi au moins autant 
que pour nos voisins. Dans tous les domaines de l'entreprise congolaise, cette belle et féconde activité a porté des fruits merveilleux. La moisson qui s'annonce est faite pour réjouir; qui ne serait heureux de le constater? N'empêche qu'il reste beaucoup à faire et c'est ce qui reste à montrer.

Sur un point l'accord semble fait dans l'opinion publique au regard de l'œuvre congolaise. Elle n'est plus, à quelques exceptions près, considérée comme une aventure d'où il soit impossible de tirer des avantages concrets. Bien au contraire, les hommes d'affaires portent leurs efforts vers l'Afrique mystérieuse avec une activité qui s'accentue et s'accélère de jour en jour, à telle enseigne que, dans certains cas, il a été permis de parler "d'engouement \%. Quand bien même des échecs partiels se produiraient, amenant à leur suite quelque désillusion, et qu'il serait acquis que d'aucuns ont manqué de prudence et de réflexion, le branle n'en est pas moins donné à des travaux et à des entreprises qui ont une valeur intrinsèque indépendante des hasards de la spéculation. Les mines d'or du Transvaal, les champs glacés du Klondyke ont provoqué beaucoup de ruines individuelles; cela n'empêche que les uns et les autres constituent des sources immenses d'enrichissement pour des pays entiers, transformant et décuplant leurs capacités industrielles et commerciales.

En ce qui concerne le développement des intérêts matériels, ou, si l'on veut, la pénétration réciproque des intérêts métropolitains et coloniaux, la situation au Congo est donc satisfaisante. La progression constante 


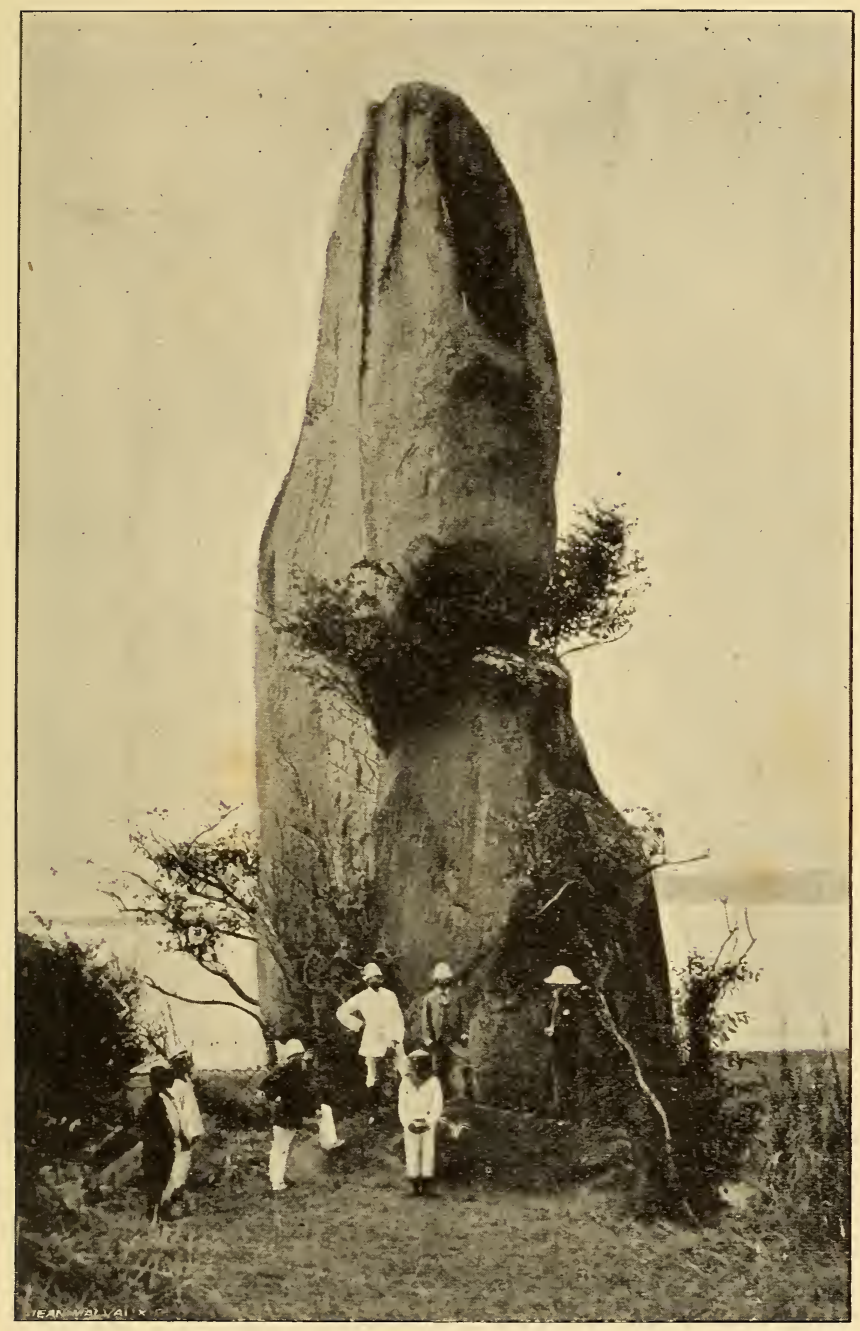

Le monolithe près de Boma. (Phot. du capitaine Deuster.) 

et rapide dans le chiffre des transactions - telle que n'en montre peut-être aucune entreprise similaire est de nature à commander la confiance.

C'est à un autre point de vue qu'il faut se placer pour désirer mieux que ce qui existe actuellement.

Il ne suffit pas que certaines catégories de citoyens, se rendant compte du profit considérable à réaliser par un pays industriel dans les affaires coloniales, sachent payer de leur personne et de leur bourse.

Sans doute, les bases économiques sont, en pareille matière, parmi les plus solides. La Belgique, pays producteur par excellence, doit rechercher l'ouverture de nouveaux débouchés. Il importe au plus haut point qu'elle place, partout où il se peut, les articles de ses manufactures et qu'elle sache alimenter celles-ci de matières premières susceptibles de transformations industrielles rémunératrices. A titre d'exemples, il suffira de citer, à l'exportation, les tissus, à l'importation, le caoutchouc.

Mais quand nous aurons reconnu que les statistiques fournissent des arguments précieux aux " coloniaux ", et que la politique d'expansion - comme cela doit être dans notre pays - obéit aux directions de la saine raison commerciale bien plus qu'elle ne répond aux appels d'une vaine gloriole, encore faudra-t-il se demander si cette politique n'implique pas de soucis plus élevés. N'est-elle pas, au premier chef, l'affirmation de la personnalité nationale? La nation, personne morale, qui vit de tout l'élan des cœurs et des idées, infiniment plus encore que de la prospérité que lui amè- 
nent en fractions individuelles le labeur et l'épargne de ses membres.

A cet égard il convient d'observer que si un homme peut fonder une grande œuvre, - les puissantes initiatives émanent même toujours d'un seul, - pour maintenir et développer l'œuvre, il faut une énergie collective, un peuple. Il ne s'agit pas ici, bien entendu, de l'action gouvernementale, dont les ressorts se prêtent à des combinaisons variées à étudier à loisir, mais de cette poussée d'intérêt, d'influence, de volonté inconsciente et généreuse qui se manifeste par l'opinion publique. Tant que le Congo ne retiendra l'attention, voire l'attachement, que de ceux qui y possèdent des intérêts directs, sa cause ne sera pas nationale au point de vue belge. Aussi rapides qu'on suppose ses progrès, aussi considérable l'élargissement de ses ondes de prospérité, de longtemps encore il ne formera dans le mouvement extérieur de la Belgique qu'un appoint assez faible. Les concours indispensables doivent venir de ceux qui ne gagnent rien directement et qui ne songent même pas à gagner quelque chose. C'est assurément la grande majorité. Et elle répond, en l'état actuel, quand on lui représente les bénéfices réalisés par tel groupe déterminé de concitoyens - industriels, commerçants, ouvriers : "Qu'importe! cela ne me touche pas ", quand en outre elle n'invoque pas comme un passedroit odieux la méconnaissance de tel ou tel grief local.

A cet égoïsme, il est urgent d'opposer l'esprit de solidarité nationale, fait des impulsions les plus désintéressées. Mais à son tour celui-ci ne s'éveille et ne prend 
conscience de lui que dans la mesure où on lui prépare les voies. Or, en Belgique on a toujours pris un soin exagéré de séparer la cause congolaise de celle du pays; on a insisté avec un luxe inoui de précautions sur l'inexistence de liens réels entre ce dernier et la contrée d'outre-mer que le monde entier appelle aujourd'hui le Congo belge; on a poussé aussi loin qu'il était possible la notion d'autonomie et d'indépendance de l'Etat nouveau, encore qu'elle contînt une part indéniable de fiction juridique, puisque ce sont des hommes et des capitaux procurés par la Belgique, notre sang et notre or, qui ont fait le Congo ce qu'il est. Étant parti de là, il advint que l'État Indépendant fortifia graduellement ses organes de vie propres, au point de faire croire à d'aucuns - à tort à notre sens - qu'il est animé, de son côté, comme d'un mouvement particulariste. Et la sécurité apparente qu'en retire la Belgique, cache en réalité un sérieux péril.

Dans la société des nations, notre pays jouit, à juste titre, d'une haute considération. Ses traditions gouvernementales et administratives défient toute appréciation malveillante. Les adversaires du Congo - il y en a en maints endroits - sont, au contraire, beaucoup plus à l'aise pour dénigrer une œuvre dont la Belgique, comme telle, ne revendiquerait que par personne interposée la paternité. L'un de leurs arguments favoris est d'alléguer qu'elle n'entend pas être puissance colonisatrice, qu'elle ne tient pas au Congo. Et en même temps il ne se font pas faute d'attaquer les actes de nos compatriotes, qui sont donc devenus autres, parce qu'ils sont au service de l'État Indépendant. 
D'autre part, en voulant réduire au minimum les points de contact, et, si j'ose ainsi dire, de suture entre la Belgique et le Congo, l'on perd de vue que les greffes de la civilisation risquent de devenir insuffisantes, sinon inefficaces. Le jeune État en est, on le sait, à son premier stade de développement. A mesure qu'il grandit, il lui faut des aides nouvelles, des secours en forces fraîches. Les-officiers et sous-officiers détachés de l'armée belge ne pourront toujours faire face à des besognes qui vont se multipliant, qui échappent, le cas échéant, à leur compétence, sans compter les éventualités qui obligeraient brusquement l'État à se priver de leur concours.

Il reste à former, à entretenir un personnel civil, à poursuivre l'extension dinstitutions administratives et judiciaires à des territoires immenses. Où sont les organes qui faciliteraient le passage à un autre régime, les écoles notamment où devraient s'enseigner les branches étonnamment riches et variées de la science coloniale? Qu'on ne se laisse pas aller trop tôt à la satisfaction des grandes choses accomplies. La propagande systématique, le travail d'initiation, en pays belge, sont à peine ébauchés. Sous cet aiguillon seulement se formera cette opinion publique qui est dans nos vœux, ardente et réfléchie à la fois, sachant discerner et sachant agir, comme cela se voit dans les rangs profonds de la démocratie anglaise.

Qu'elle le veuille ou non, la Belgique est désormais garante, devant l'histoire, des suites de l'entreprise congolaise. Ce serait en vérité pitié, si après tant d'hé- 
roïques et heureux efforts, l'œuvre venait à être compromise ou à passer en d'autres mains, faute d'avoir été soutenue comme il convenait par le pays premier intéressé à sa réussite. Mais quoi ! ces appréhensions seront vaines. A l'aube indécise a succédé le grand jour, le but apparaît en pleine lumière, bien fait pour solliciter le meilleur de nos énergies patriotiques : C'est le prolongement au delà de l'océan d'une métropole manifestement trop à l'étroit chez elle, c'est la colonie fortifiant et grandissant une mère patrie également chère à tous les bons citoyens. 



\section{TABLE DES MATIÈRES}

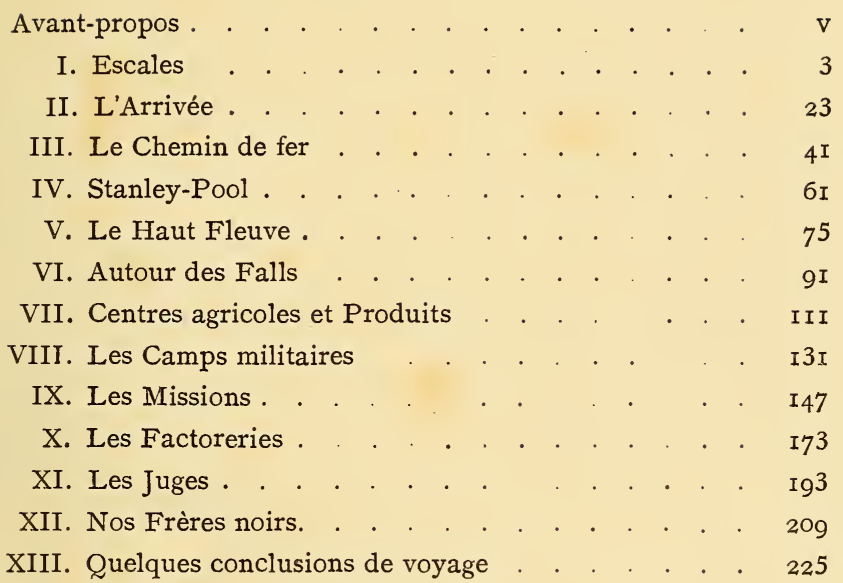


Bruxelles. - Imp. Ve Monnom, 32, rue de l'Industrie. 





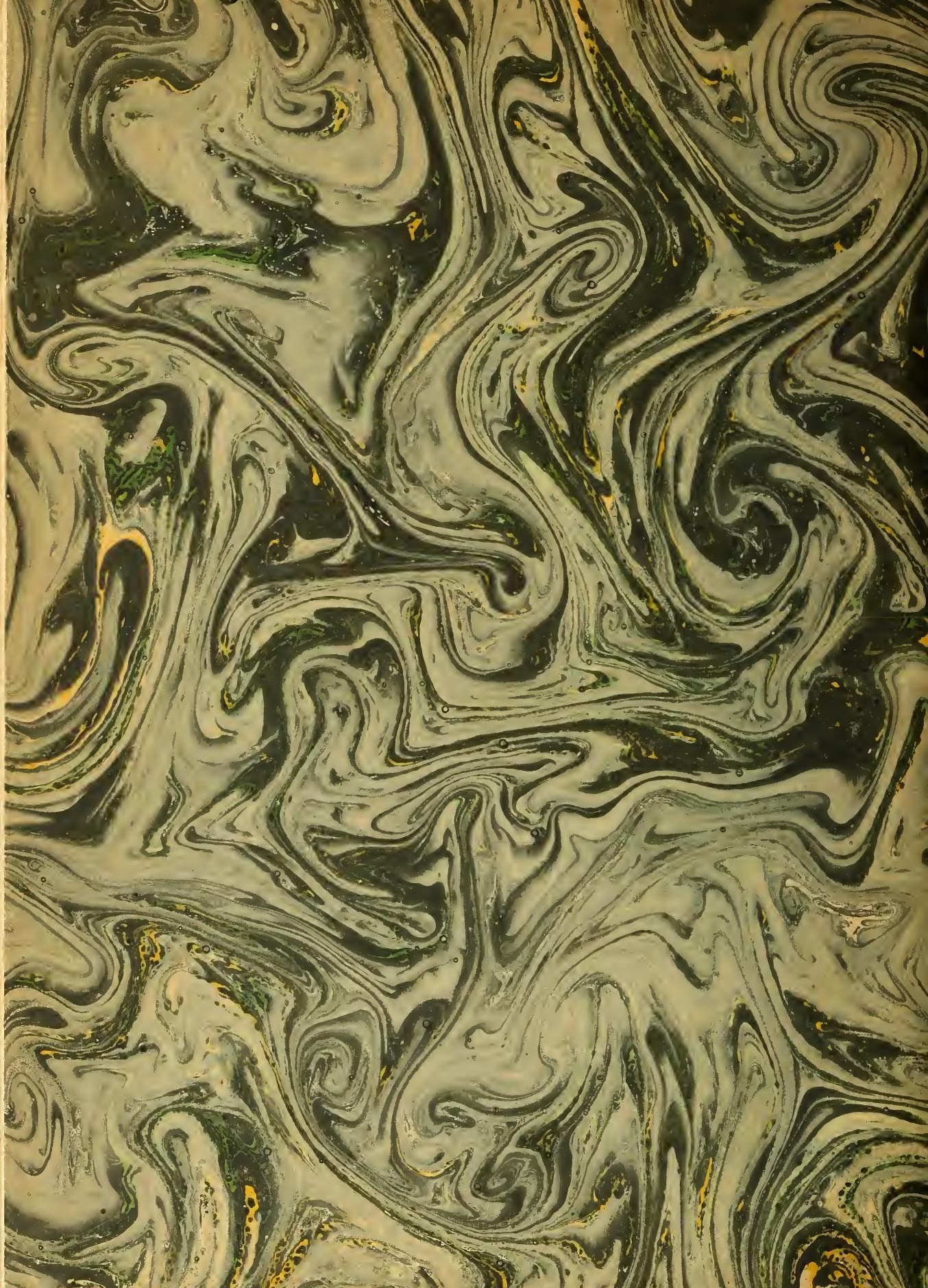




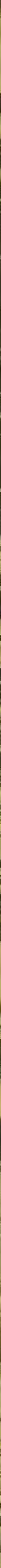


